\title{
Development of a Portal Imager and of Tools for Radiation Treatment Verification
}

\author{
Hui Wang \\ Department of Physics \\ McGill University, Montreal \\ July, 1996
}

A Thesis Submitted to the Faculty of Graduate Studies and Research in Partial Fulfillment of the Requirements of the Degree of Doctor of Philosophy

CHui Wang 1996 
National Library

of Canada

Acquisitions and

Bibliographic Services Branch

395 Wellington Streel

Ottawa. Ontario

KIA ONA
Bibliothéque nationale uu Canada

Direction des acquisitions et des services biblingraphiques

395, ne Wel.ington

Ottawa (Ontario)

Vour tive votre reterence

Our five Notre Peterence

The author has granted an irrevocable non-exclusive licence allowing the National Library of Canada to reproduce, loan, distribute or sell copies of his/her thesis by any means and in any form or format, making this thesis available to interested persons.

The author retains ownership of the copyright in his/her thesis. Neither the thesis nor substantial extracts from it may be printed or otherwise reproduced without his/her permission.
L'auteur a accordé une licence irrévocable et non exclusive permettant à la Bibliothèque nationale du Canada de reproduire, prêter, distribuer ou vendre des copies de sa thèse de quelque manière et sous quelque forme que ce soit pour mettre des exemplaires de cette thèse à la disposition des personnes intéressées.

L'auteur conserve la propriété du droit d'auteur qui protège sa thèse. Ni la thèse ni des extraits substantiels de celle-ci ne doivent être imprimés ou autrement reproduits sans son autorisation.

ISBN $\quad 0-612-19783-2$ 


\section{Abstract}

A prototype electrostatic imager has been developed for megavoltage portal imaging in radiation therapy. The imager utilizes amorphous selenium (a-Se) with a front meta! plate as the imager receptor and a high resolution voltmeter probe for image readout. Imaging characteristics of a-Se have been investigated theoretically through Monte Carlo simulations, and experimentally by measuring radiation discharging curves and phantom tests. The results of this study have shown that the prototype imager has high sensitivity, good spatial resolution and low noise level. Our study also reveals the potential of electrostatic imaging with metal/a-Se in megavoltage imaging.

Two computer algorithıns have also been developed for automatic segmentation and contrast-enhancement of digital portal images, and for radiation field shape verification. Based on a priori knowledge of the properties of portal images, the segmentation and contrast-enhancement algorithm employs multiple criteria and dynamic reasoning to achieve optimal segmentations of individual images, and has been proved to be accurate, robust and fast. The algorithm for radiation field shape verification is an adaptation of the chamfer matching technique to a specific application: matching closed contours. By incorporating geometric features of the radiation field and using a simple minimization method which is more specific to this task, the algorithm appears to be able to improve the matching results of the standard method. 


\section{Résumé}

Un nouveau détecteur électrostatique a èté çonu pour l'imagerie numérique de vérification lors de la radiothérapie effectué avec des faisceaux de photons à haute énergie. Ce détecteur consiste en une plaque de sélénium amorphe à haute tension placée derrière une feüille de métal. Une fois irradièe, la lecture de la tension locale sur la plaque de sélénium se fait par moyen d'une sonde de haute résolution. Les propriétés radiologiques du sélénium amorphe ont été examiné théoriquement à l'aide de simulations Monté Carlo, et expérimentellement en mesurant les courbes de décharge et en effectuant des tests avec des fantômes. Nous avons constaté que notre détecteur est très sensible, peu contaminè par le bruit, et possède une bonne résolution spatiale. Ceci témoigne du grand potentiel de l'imagerie électrostatique avec le métal/sèlénium amorphe pour la vérification en radiothérapie.

Un premier algorithme informatique a èté développé pour la segmentation automatique et le rehaussement du contraste des inages. Un deuxième algorithme a été développé pour la vérification de la forme des champs de radiation. En se basant sur les propriétés a priori des images numériques de vérification, le premier algorithme utilise des critères multiples et le raisonnement dynamique pour arriver à une segmentation optimale de l'image, rapidement et sûrement. Le deuxième algorithme adapte le procédè du "chamfer matching" au problème de l'ajustement de contours fermés. En incorporant les traits géométriques du champs de radiation et en utilisant une méthode de minimisation 
appropièe, cet algorithme parait ètre plus performante que l'algorithme conventionn:lle. 


\section{Original Contribution}

The work presented in this thesis has resulted in several published articles, abstracts and conference presentations in the past a few years. Our investigations have led to a new prototype portal imager in radiation therapy and two computer algorithms for portal image segmentation and radiation field shape verification.

Being the first of its kind that has been reported in electrostatic portal imaging, the prototype imager has been shown to have potential due to its demonstrated high sensitivity and low noise. With proper engineering, it can be expected to develop into a viable product in the near future.

The portal image segmentation algorithm introduced a new approach in radiation field extraction required for contrast enhancement and quantification of the radiated area. Based on morphological technigl!ns, the algorithm employed a dynamic approach to search the optimal segmentation for each individual portal image and therefore can accommodate very difficult situations.

We have also investigated the possibility of adapting the standard chamfer matching technique to a specific task: radiation field shape verification. Our algorithm adopted the chamfer matching principle and tailored the minimization method to seek improved performance in the context of matching closed contours, which has been demonstrated by the results. 


\section{Acknowledgment}

I would like to thank my supervisor, Dr. B. Gino Fallone, for his guidance and support during the past four years. The steady progress of my research which has led to the completion of this thesis is the direct result of his excellent supervision.

As a graduating student, I would like to extend my gratitude to the staff of the Medical Physics Unit, especially the director, Dr. Ervin Podgorsak, for working so hard to make this educational program one of the bests in North America. The high quality of the education that we received at McGill will undoubtedly benefit all our fellow graduates in our cirreers.

Being a member of the portal imaging group, I have always enjoyed the congenial environment. The inspiring discussions and fruitful collaborations with lan Crooks, M.Sc., Tony Falco, M.Sc., and Dimitri Hristov, B.Sc. will always be remembered. I sincerely thank Tony for his contribution to the a-Se imager project. Working in the medical physics lab has also given me the opportunity to make good friends with the "dogs" and the "cat": Brennan MacDonald, Ph.D., Ian Crooks, M.Sc., Brian Keller, M.Sc., Wiltiam Parker, M.Sc., Corey Zankowski, M.Sc., and Chantal Audet, Ph.D.. Their friendship has made this period of student life very enjoyable. I also want to thank my good friends, Andrew Lukban, M.Sc., and Arthur Curtin-Savard, M.Sc., for helping me going through many difficult times. 
The research presented in this thesis has been financially supported in part by the Sterling Winthrop Imaging Research Institute, the Medical Research Council of Canada, Picker International Inc., and Noranda Advanced Materials Inc.. The Teaching Assistantship from the Physics Department is also gratefully acknowledged.

This thesis is dedicated to my parents. Without their love and understanding, it would not have been accomplished. 


\section{Table of Contents}

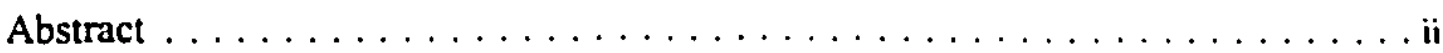

Rèsumè $\ldots \ldots \ldots \ldots \ldots \ldots \ldots \ldots \ldots \ldots \ldots \ldots \ldots \ldots \ldots \ldots \ldots \ldots$

Original Contribution $\ldots \ldots \ldots \ldots \ldots \ldots \ldots \ldots \ldots \ldots$

Acknowledgment $\ldots \ldots \ldots \ldots \ldots \ldots \ldots \ldots \ldots \ldots \ldots \ldots \ldots$ vi

List of Figures $\ldots \ldots \ldots \ldots \ldots \ldots \ldots \ldots \ldots \ldots \ldots \ldots \ldots \ldots \ldots$

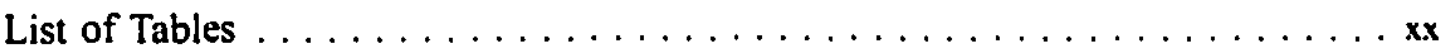

Chapter 1 Introduction $\ldots \ldots \ldots \ldots \ldots \ldots \ldots \ldots \ldots$

$\$ 1.1$ Portal Imaging in Radiation Therapy $\ldots \ldots \ldots \ldots$

$\S 1.2$ Electronic Portal Imaging Devices (EPIDs) $\ldots \ldots \ldots \ldots$

$\$ \$ 1.2 .1 \quad$ Fluoroscopic Systems $\ldots \ldots \ldots \ldots \ldots \ldots \ldots$

$\S \S 1.2 .2$ Matrix Ionization Chamber Systems . . . . . . . . . 5

$\S \S 1.2 .3 \quad$ Alternative EPIDs $\ldots \ldots \ldots \ldots \ldots \ldots$

$\S 1.3$ Treatment Setup and Verification $\ldots \ldots \ldots \ldots$

$\$ 1.4$ Thesis Organization $\ldots \ldots \ldots \ldots \ldots \ldots$

References $\ldots \ldots \ldots \ldots \ldots \ldots \ldots \ldots \ldots \ldots \ldots \ldots \ldots \ldots$

Chapter 2 Imaging Characteristics of Metal/a-Se: Monte Carlo Studies . . . . 20

$\S 2.1$ Introduction $\ldots \ldots \ldots \ldots \ldots \ldots \ldots \ldots \ldots \ldots \ldots \ldots \ldots$

$\S 2.2$ Amorphous Selenium (a-Se) for Portal Imaging . . . . . . . 22

$\S 2.3$ Receptor Blur and Modulation Transfer Function . . . . . . 23

$\S 2.4$ Quantum Noise and Detective Quantum Efficiency . . . . . 27

$\$ 2.5$ Monte Carlo Simulations of Energy Deposition . . . . . . . 30

$\S \S 2.5 .1 \quad$ The EGS4 Code $\ldots \ldots \ldots \ldots \ldots \ldots \ldots$ 
$\S \S 2.5 .2 \quad$ Receptor Geometry $\ldots \ldots \ldots \ldots \ldots \ldots \ldots \ldots 31$

$\S \S 2.5 .3$ Calculations of MTF $\ldots \ldots \ldots \ldots \ldots \ldots \ldots . \ldots \ldots 2$

$\S \S 2.5 .4$ Calculations of Absorption Efficiency and DQE . . . . . 33

$\$ 2.6 \quad$ Results .......................... 34

$\S \S 2.6 .1$ Modulation Transfer Function . . . . . . . . . . 34

$\S \S 2.6 .2$ Quantum Absorption Efficiency . . . . . . . . . 46

$\S \S 2.6 .3$ Detector Response . . . . . . . . . . . . . . 47

$\S \S 2.6 .4$ Statistical Factor $\ldots \ldots \ldots \ldots \ldots \ldots \ldots \ldots . \ldots 4$

$\S \S 2.6 .5$ Detective Quantum Efficiency .............. 5t

$\S 2.7$ Conclusions $\ldots \ldots \ldots \ldots \ldots \ldots \ldots \ldots \ldots \ldots \ldots$

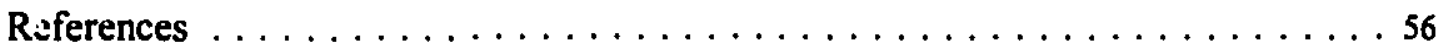

Chapter 3 Sensitivity and Contrast-Detaii Studies $\ldots \ldots \ldots \ldots \ldots \ldots 58$

$\$ 3.1$ Introduction $\ldots \ldots \ldots \ldots \ldots \ldots \ldots \ldots \ldots \ldots$

$\$ 3.2$ System Overview. . . . . . . . . . . . . . . . . . 59

$\S \S 3.2 .1$ The Detector Unit $\ldots \ldots \ldots \ldots \ldots \ldots \ldots \ldots 6$

$\S \S 3.2 .2$ The Electronics System. . . . . . . . . . . . 61

$\S \S 3.2 .3$ The Host Computer $\ldots \ldots \ldots \ldots \ldots \ldots \ldots \ldots 63$

$\S 3.3$ Electrostatic Image Formation $\ldots \ldots \ldots \ldots \ldots \ldots \ldots 6$

$\S \S 3.3 .1 \quad$ Charging $\ldots \ldots \ldots \ldots \ldots \ldots \ldots \ldots \ldots \ldots \ldots$

$\$ \S 3.3 .2 \quad$ Receptor Orientation. . . . . . . . . . . . . . 64

$\S 3.4$ Image Acquisition $\ldots \ldots \ldots \ldots \ldots \ldots \ldots \ldots 6$

$\S 3.5$ Sensitivity Curve Measurement and Modelling ........65

$\S \S 3.5 .1 \quad$ Radiation Discharging $\ldots \ldots \ldots \ldots \ldots \ldots \ldots 6$

$\S \S 3.5 .2$ Monoenergetic Photons . . . . . . . . . . . . . 67 
$\$ \$$ 3.5.3 Polyenergetic Photons . . . . . . . . . . . . 76

$\$ 3.6$ Phantom Tests $\ldots \ldots \ldots \ldots \ldots \ldots \ldots$

$\$ 3.7$ Conclusions $\ldots \ldots \ldots \ldots \ldots$

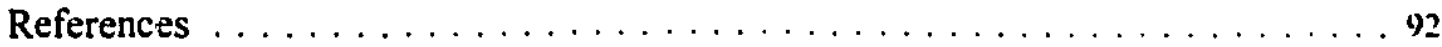

Chapter 4 Segmentation of Portal Images $\ldots \ldots \ldots \ldots \ldots \ldots$

$\S 4.1 \quad$ Introduction $\ldots \ldots \ldots \ldots \ldots$

$\$ \$ 4.1 .1 \quad$ Objectives $\ldots \ldots \ldots \ldots \ldots \ldots$

$\$ \$ 4.1 .2 \quad$ Scope $\ldots \ldots \ldots \ldots \ldots \ldots$

$\S 4.2 \quad$ Materials and Methods $\ldots \ldots \ldots \ldots \ldots \ldots$

$\$ \$ 4.2 .1 \quad$ Image Processing System $\ldots \ldots \ldots \ldots$

$\$ \$ 4.2 .2 \quad$ Image Acquisition $\ldots \ldots \ldots \ldots \ldots \ldots$

$\S 4.3 \quad$ Mathematical Morphology . . . . . . . . . . . 103

$\$ \$ 4.3 .1 \quad$ Fundamental Operations . . . . . . . . . . . . . 104

$\S \S$ 4.3.2 Complex Operations and Algorithms . . . . . . . 108

$\$ \$ 4.3 .3 \quad$ Digital Morphology $\ldots \ldots \ldots \ldots \ldots \ldots \ldots$

$\$ \$ 4.3 .4 \quad$ Gray Scale Morphology . . . . . . . . . . . . 114

$\$ \$ 4.3 .5 \quad$ Morphological Gradient $\ldots \ldots \ldots \ldots \ldots \ldots$

$\S 4.4 \quad$ Edge Detection $\ldots \ldots \ldots \ldots \ldots \ldots \ldots \ldots$

$\$ \$ 4.4 .1 \quad$ Gradient Operators . . . . . . . . . . . . . 117

$\$ \S 4.4 .2 \quad$ Optimal Edge Detectors $\ldots \ldots \ldots \ldots \ldots \ldots$

$\S \S 4.4 .3 \quad$ Morphological Edge Detectors . . . . . . . . . 122

$\S 4.5 \quad$ Algorithm Development . . . . . . . . . . . . 124

$\S \S 4.5 .1 \quad$ Basic Approach $\ldots \ldots \ldots \ldots \ldots \ldots \ldots \ldots \ldots$

$\$ \S 4.5 .2 \quad$ Feature Extraction $\ldots \ldots \ldots \ldots \ldots \ldots \ldots$ 
$\S \S 4.5 .3 \quad$ Edge Localization $\ldots \ldots \ldots \ldots \ldots \ldots \ldots \ldots$

$\S \S 4.5 .4$ Automation of Segmentation ............ 138

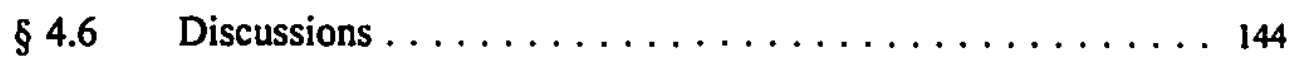

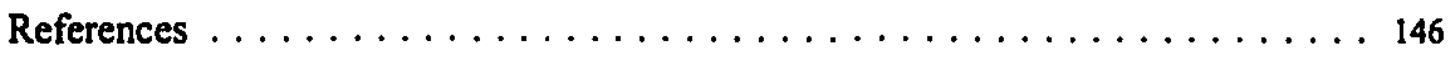

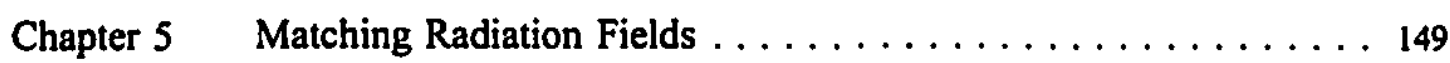

$\S 5.1$ Introduction $\ldots \ldots \ldots \ldots \ldots \ldots \ldots \ldots \ldots \ldots \ldots$

$\S 5.2 \quad$ Materials and Methods . . . . . . . . . . . . . 152

$\S \S 5.2 .1 \quad$ Edge Distance Map Generation . . . . . . . . 152

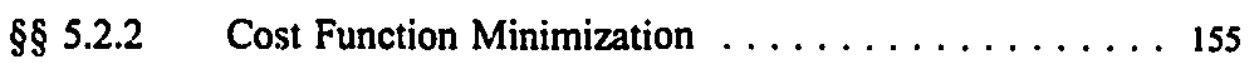

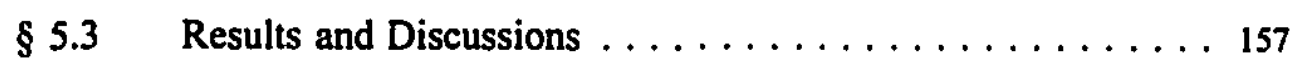

$\$ \S 5.3 \quad$ Minimization Approach $\ldots \ldots \ldots \ldots \ldots \ldots \ldots 158$

$\S \S 5.3 .2 \quad$ Computation Speed $\ldots \ldots \ldots \ldots \ldots \ldots \ldots \ldots 2$

$\S \S 5.3 .3$ Effects of Cost Functions $\ldots \ldots \ldots \ldots \ldots \ldots \ldots 162$

$\S \S$ 5.3.4 Effects of Distance Transformations . . . . . . . 164

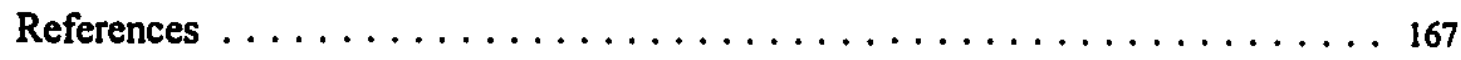

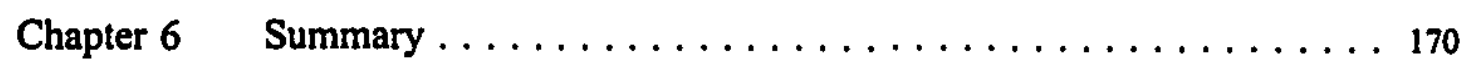

$\S 6.1 \quad$ Electrostatic Portal Imaging $\ldots \ldots \ldots \ldots \ldots \ldots \ldots 171$

$\$ \S 6.1 .1 \quad$ Amorphous Selenium $\ldots \ldots \ldots \ldots \ldots \ldots \ldots \ldots 171$

$\$$ 6.1.2 Front Metal Plate $\ldots \ldots \ldots \ldots \ldots \ldots \ldots \ldots . \ldots \ldots$

$\S \S 6.1 .3$ Future Work $\ldots \ldots \ldots \ldots \ldots \ldots \ldots \ldots \ldots$

$\S 6.2$ Portal Image Segmentation $\ldots \ldots \ldots \ldots \ldots \ldots \ldots . \ldots \ldots$

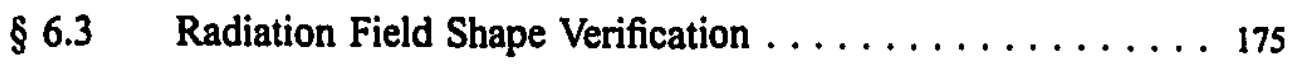

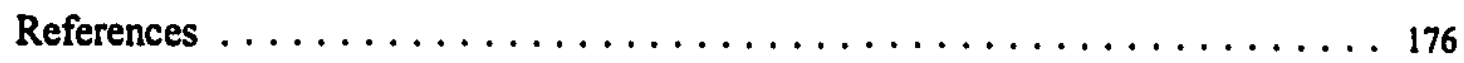

Appendix A Minimization by Bracketing $\ldots \ldots \ldots \ldots \ldots \ldots \ldots \ldots$ 
Bibliography $\ldots \ldots \ldots \ldots \ldots \ldots \ldots \ldots \ldots \ldots \ldots \ldots \ldots \ldots \ldots$ 


\section{List of Figures}

Figure 2.1

Figure 2.2

Figure 2.3

Figure 2.4

Figure 2.5

Figure 2.6

Figure 2.7

Figure 2.8

Figure 2.9

Figure 2.10

Figure 2.11

Figure 2.12
Geometry of the line spread function simulation $\ldots \ldots \ldots 32$

Geometry of the energy deposition simulation $\ldots \ldots \ldots . \ldots 34$

The modulation transfer functions of a $2 \mathrm{~mm} \mathrm{Al} / 0.15 \mathrm{~mm}$ a-Se receptor at various photon energies. .35

The modulation transfer functions of a $2 \mathrm{~mm} \mathrm{Al} / 0.3 \mathrm{~mm}$ a-Se receptor at various photon energies. $\ldots \ldots \ldots \ldots \ldots$ The modulation transfer functions of a $2 \mathrm{~mm} \mathrm{Al} / 0.5 \mathrm{~mm}$ a-Se receptor at various photon energies. . . . . . . . . 37 The modulation transfer functions of a $1 \mathrm{~mm} \mathrm{Cu} / 0.3 \mathrm{~mm}$ a-Se receptor at various photon energies. $\ldots \ldots \ldots \ldots \ldots$ The modulation transfer functions of a $0.3 \mathrm{~mm} \mathrm{a-Se} / 1 \mathrm{~mm} \mathrm{Cu}$ receptor at various photon energies. . . . . . . . . 39 The modulation transfer functions of four receptor at incident photon energy of $1 \mathrm{MeV} . \ldots \ldots \ldots \ldots$ The modulation transfer functions of four receptor at incident photon energy of $2 \mathrm{MeV} \ldots \ldots \ldots \ldots \ldots \ldots$ The modulation transfer functions of four receptor at incident photon energy of $3 \mathrm{MeV} \ldots \ldots \ldots \ldots \ldots \ldots$ The modulation transfer functions of four receptor at incident photon energy of $4 \mathrm{MeV} \ldots \ldots \ldots \ldots \ldots \ldots$ The modulation transfer functions of four receptor at incident photon energy of $5 \mathrm{MeV} . \ldots \ldots \ldots \ldots \ldots \ldots$ xiii 
Figure 2.13

Figure 2.14

Figure 2.15

Figure 2.16

Figure 2.17

Figure 3.1

Figure 3.2

Figure 3.3

Figure 3.4

Figure 3.5

Figure 3.6
The modulation transfer functions of four receptor at incident photon energy of $6 \mathrm{MeV} . \ldots \ldots \ldots \ldots \ldots \ldots \ldots \ldots$

Quantum absorption efficiencies of four receptors at various incident photon energies. . . . . . . . . . . . . 48

Detector response of four receptors at different various incident photon energies. . . . . . . . . . . . . . . . . . 49

Statistical factors of four receptors at different incident photon energies. ..........................50

Zero spatial frequency detective quantum efficicincy of four receptors at various incident photon energies. . . . . . . . 52 Major components of the prototype portal imager. . . . . 60 Schematics of the detector unit (Courtesy of Tony Falco). . . 62 Sensitivity curves of a $2 \mathrm{~mm} \mathrm{Al} / 0.15 \mathrm{~mm}$ a-Se receptor measured on a T780 Cobalt Unit with the front plate and back plate configurations. The dose rate at the receptor is $37 \mathrm{cGy} / \mathrm{min} . ~ . ~ 70$ Sensitivity curves of a $2 \mathrm{~mm} \mathrm{Al} / 0.3 \mathrm{~mm}$ a-Se receptor measured on a T780 Cobalt Unit with the front plate and back plate configurations. The dose rate at the receptor is $37 \mathrm{cGy} / \mathrm{min} . ~ .71$ Sensitivity curves of a $2 \mathrm{~mm} \mathrm{Al} / 0.5 \mathrm{~mm}$ a-Se receptor measured on a T780 Cobalt Unit with the front plate and back plate configurations. The dose rate at the receptor is $37 \mathrm{cGy} / \mathrm{min}$. . . 72 Sensitivity curves of a $1 \mathrm{~mm} \mathrm{Cu} / 0.3 \mathrm{~mm}$ a-Se receptor measured on a T780 Cobalt Unit with the front plate and back plate configurations. The dose rate at the receptor is $37 \mathrm{cGy} / \mathrm{min} . ~ . ~ .73$ 
Figure 3.7

Figure 3.8

Figure 3.9

Figure 3.10

Figure 3.11

Figure 3.12

Figure 3.13
The sensitivity curves of four different a-Se receptors measured on a T780 Cobalt Unit. Dose rate at the receptor is $37 \mathrm{cGy} / \mathrm{min} . ~ .74$

Sensitivity curves of a $2 \mathrm{~mm} \mathrm{Al} / 0.15 \mathrm{~mm}$ a-Se receptor measured on a Clinac $2300 \mathrm{C} / \mathrm{D}$ with the front plate and back plate configurations. The dose rate at the receptor is $0.3 \mathrm{cGy} / \mathrm{MU}$. . . 78

Sensitivity curves of a $2 \mathrm{~mm} \mathrm{Al} / 0.3 \mathrm{~mm}$ a-Se receptor measured on a Clinac $2300 \mathrm{C} / \mathrm{D}$ with the front plate and back plate configurations. The dose rate at the receptor is $0.3 \mathrm{cGy} / \mathrm{MU}$. . 79

Sensitivity curves of a $2 \mathrm{~mm} \mathrm{Al} / 0.5 \mathrm{~mm}$ a-Se receptor measured on a Clinac $2300 \mathrm{C} / \mathrm{D}$ with the front plate and back plate configurations. The dose rate at the receptor is $0.3 \mathrm{cGy} / \mathrm{MU}$. . 80

Sensitivity curves of a $1 \mathrm{~mm} \mathrm{Cu} / 0.3 \mathrm{~mm}$ a-Se receptor measured on a Clinac $2300 \mathrm{C} / \mathrm{D}$ with the front plate and back plate configurations. The dose rate at the receptor is $0.3 \mathrm{cGy} / \mathrm{MU} . .81$

The sensitivity curves of four different a-Se receptors measured on a Clinac $2300 \mathrm{C} / \mathrm{D}$. The dose rate at the receptor is 0.3 cGy/MU. . . . . . . . . . . . . . . . . . . 82

Images of a contrast-detail phantom acquired with a $1 \mathrm{~mm}$ $\mathrm{Cu} / 300 \mu \mathrm{m}$ a-Se receptor on a Clinac $2300 \mathrm{C} / \mathrm{D}$ linear accelerator by using the $6 \mathrm{MV}$ beam. Top: front plate image; bottom: back plate image. $\ldots \ldots \ldots \ldots \ldots \ldots \ldots . \ldots 88$ 
Figure 3.14

Figure 3.15

Figure 4.1

Figure 4.2

Figure 4.3

Figure 4.4

Figure 4.5
Images of a contrast-detail phantom acquired with a fluoroscopic EPID (top left), a matrix ionization chamber EPID (top right) and our prototype imager (bottom) on a Clinac $2300 \mathrm{C} / \mathrm{D}$ linear accelerator by using the $6 \mathrm{MV}$ beam (Courtesy of Tony Falco). . 89 Image of a head phantom acquired with a $1.5 \mathrm{~mm} \mathrm{Cu} / 300 \mu \mathrm{m}$ a-Se receptor on a Clinac $2300 \mathrm{C} / \mathrm{D}$ linear accelerator by using the $6 \mathrm{MV}$ beam (Courtesy of Tony Falco). . . . . . . . 90

(a) original image, (b) structuring element, (c) solid objects are the eroded objects, the outer contour represents the original objects, (d) solid objects are the originals, the outer contour represents the dilated objects. . . . . . . . . . . . 107

(a) original image, (b) structuring element, (c) opening of the original image, (d) closing of the original image. . . . . . 110 Illustration of the hole-filling operation. (a) original image, (b) complement of the original image, (c) image containing a rectangular frame on the image border, (d) intersection of (b) with (c) after some iterations, (e) the convergence of the iterations, (f) complement of (e) representing completion of the hole-filling operation. . . . . . . . . . . . 112

Performance of three different edge detectors on a double exposure portal image. (a) The original portal image; (b) Sobel, threshold=1; (c) Canny-Deriche, threshold=4; (d) Mgradient, threshold $=6 . \ldots \ldots \ldots \ldots \ldots \ldots \ldots \ldots$ Contamination of gradient by anatomical variations. . . . . 127 xvi 
Figure 4.6

Figure 4.7

Figure 4.8

Figure 4.9

Figure 4.10

Figure 4.11

Figure 4.12
Illustration of the basic approach in the segmentation algorithm. Top left: original portal image; top right: binary edge image after proper thresholding of the gradient of the original image; bottom left: complement of the binary edge image; bottom right: the object corresponding to the radiation field. . . . . 129 Illustration of the "label" operation on a binary image. Top: binary image; bottom: labeled image. . . . . . . . . . 131 Illustration of the procedure of field border placement. Top left: the field mask before enlargement; top right: the field mask after five dilations, bottom left: the field mask after five erosions; bottom right: contours of the original, enlarged and reduced field masks overlaid on the original image. . . . . 133 Typical plot of average grey scale values versus the number of dilations/erosions of the data and fitting which resulted from Eq. (4.56) shown as solid line. Also depicted are the various fitting parameters. On the horizontal axis, the negative and positive numbers correspond to the number of erosions and dilations, respectively.

(a) Histogram of the correlation coefficient obtained from the curve fitting among 29 portal images; (b) Parameter $b$ obtained from the curve fitting among 29 portal images. . . . . . . 136 Flowchart of the mainstream of the segmentation and contrast enhancement procedure. . . . . . . . . . . . . . . 137 Flowchart of the first step of the segmentation procedure. . . 139 
Figure 4.13

Figure 4.14

Figure 5.1

Figure 5.2

Figure 5.3 (a) original image, (b) binary edge image at the threshold value reached by the first approximation, (c) labeled complement of (b), (d) object image at the optimal threshold value, (e) treatment field mask made from the central object in (d), ( $f$ ) portal image enhanced with SHE $\ldots \ldots \ldots \ldots \ldots \ldots \ldots 14$ Flowchart of the second step of the segmentation procedure. . 142 Illustration of procedure for chamfer matching: (a) Simulator image; (b) Portal image; (c) Prescribed field contour obtained from (a); (d) Treatment field contour extracted from (a); (e) Edge distance map generated from (c); (f) After (d) is transformed with a trial set of parameters, it is overlaid on top

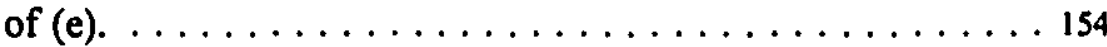

Flowchart of the comparison test between the simplex method and successive minimization along coordinate directions (S.M.A.C.D)

Comparison of final average edge distances achieved with different approaches. (a) successive line minimization versus the simplex method with "chessboard" edge distance maps and arithmetic average edge distance as cost function. (b) arithmetic versus root of mean square average edge distance as cost function when "chessboard" edge maps and successive line minimization were used. (c) "chessboard" versus "5-7-11" edge maps when arithmetic average edge distance as cost function was minimized successive line minimization. . . . . . 161 
Figure A.1

Illustration of the bracketing process for minimizing a one

dimensional function. . . . . . . . . . . . . . 177 


\section{List of Tables}

Table 3.1

Table 3.2

Table 3.3

Table 3.4

Table 3.5

Table 3.6

Table 5.7
Radiation times required for complete discharge of different receptors on T780 Cobalt Unit and correlation coefficients obtained from the modelling. $\ldots \ldots \ldots \ldots \ldots \ldots$ Relative $x$-ray sensitivity of receptors with a $2 \mathrm{~mm} \mathrm{Al} \mathrm{front}$ plate. . . . . . . . . . . . . . . . . .

Relative x-ray sensitivity of receptors with different front metal

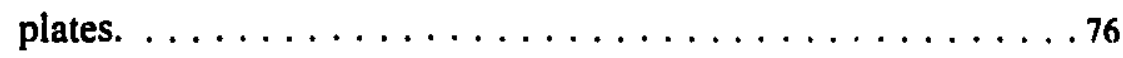

Relative $\mathrm{x}$-ray sensitivity of a receptor with front and back metal plates. . . . . . . . . . . . . . . . . 77

Monitor units required for complete discharge of different receptors on Clinac $2300 \mathrm{C} / \mathrm{D}$ and correlation coefficients obtained from the modelling. . . . . . . . . . . . . 84 The sizes and depths of the holes on the contrast-detail phantom. . . . . . . . . . . . . . . . . .87

Numbers of iterations for reaching convergence in successive line minimization along coordinate directions ( $\left.N_{\text {S.M.A.C.D. }}\right)$ and the downhill simplex method $\left(N_{\text {simplex }}\right)$. The average difference $\overline{\left(N_{\text {S.M.A.C.D. }}-N_{\text {simplex }}\right)}=-3.2 \ldots \ldots \ldots \ldots 3$ 
$\S 1.1 \quad$ Portal Imaging in Radiation Therapy $\ldots \ldots \ldots \ldots \ldots$

$\S 1.2 \quad$ Electronic Portal Imaging Devices (EPIDs) $\ldots \ldots \ldots \ldots \ldots$

$\$ \S 1.2 .1 \quad$ Fluoroscopic Systems $\ldots \ldots \ldots \ldots \ldots$

$\S \S$ 1.2.2 Matrix Ionization Chamber Systems $\ldots \ldots \ldots \ldots \ldots$

$\S \S 1.2 .3 \quad$ Alternative EPIDs . . . . . . . . . . . . . 5

$\S 1.3 \quad$ Treatment Setup and Verification $\ldots \ldots \ldots \ldots \ldots$

$\S 1.4 \quad$ Thesis Organization $\ldots \ldots \ldots \ldots \ldots$ 


\section{\$ 1.1 Portal Imaging in Radiation Therapy}

After decades of development, radiation therapy has become more and more effective in controlling cancer. One of the many factors that has led to the advancement is the ongoing improvement in the precision of radiation dose delivery to the target. Most patients undergoing radiotherapy today are treated with high energy x-rays. Accurate localization of a treatment $\mathrm{x}$-ray beam to the target requires taking images with the very same beam at each port. This procedure is referred to as portal imaging or megavoltage imaging.

In one of the first reports of portal imaging practice, Hare et al acquired portal images on radiographic films for the purpose of localizing a radiotherapy patient prior to treatment. The use of portal films was found to be an important measure for achieving a high accuracy in radiation treatments. Since then, portal films has become more commonly used in radiotherapy for radiation beam positioning and radiation field shape verification. As a quality assurance procedure, portal imaging ensures accurate implementation of a treatment plan and therefore can significantly change the outcome of the treatment. In a study on localization errors for patients treated with extended mantle fields, Marks $e t a l^{2}$ reported that increasing the frequency of verification film checks from an average of nine to twenty-four per treatment course decreased the frequency of localization and field design errors from a $36 \%$ overall error rate to $15 \%$. In 1988 , a 
protocol was published by the American Association of Physicists in Medicine to serve as the guideline for portal imaging practice. ${ }^{3}$ Because of the low detection efficiency of film at therapeutic energies a metal screen is placed on top of the portal film. The metal plate is the $\mathrm{x}$-ray detector that converts the incident photons into secondary electrons that subsequently interact with the film to form the portal image. The metal plate also shields the scattered radiation from reaching the film. Droege and Bjarngard reported that the metal plate increases the overall contrast by reducing the scatter-to-primary ratio. ${ }^{4}$

Although portal films have been proven to be an effective means to ensure accurate setup of radiation treatments, they are not used for every treatment session due to a low intrinsic contrast and a lengthy developing time. In a survey conducted in 1989, Dunscombe et al reported that, on average, portal radiographs were taken for only $67 \%$ of the patients, and that among the institutions surveyed, only $57 \%$ performed routine portal imaging on all their patients undergoing radical treatment. ${ }^{5}$

\section{$\S 1.2$ Electronic Portal Imaging Devices (EPIDs)}

In recent years, a large amount of effort has been devoted to the development of electronic portal imaging devices (EPIDs) which can acquire digital images in real or near real time. EPIDs make it possible to place the beam interactively and to monitor patient motion throughout the treatment. However, since most of EPIDs reported have major limitations, only two types of systems have become commercially available to the 
radiation therapy community. These include fluoroscopic and matrix ionization chamber systems.

\section{$\S \S$ 1.2.1 Fluoroscopic Systems}

Being the most common portal imaging system, a fluoroscopic EPID employes a metal plate coated with a layer of fluorescent phosphor as the image receptor. X-rays transmitted through the patient strike the metal plate and create energetic electrons some of which will enter the phosphor layer and produce fluorescence. The fluorescent lights are then coupled to a video camera with a $45^{\circ}$ mirror and a focusing lens. Since the first fluoroscopic imaging system specifically designed for megavoltage imaging ${ }^{6}$. many researchers have developed different fluoroscopic portal imagers with different types of components in order to improve image quality. For example, Leong used a siliconintensified target (SIT) camera ${ }^{7}$, Shalev et $a l^{8}$ used both intensified CCD and SIT cameras, Visser et al ${ }^{9}$ used a CCD camera with slow scan readout capabilities, and Munro et al ${ }^{10}$ used a lead oxide tube camera specially adapted to accumulate the video signal on the lead oxide target of the tube for lengthy periods. This type of system generally has a high spatial and contrast resolution. However, the signal to noise ratio is limited by the low efficiency of light collection (1\%). ${ }^{1}$ After years of improvement, fluoroscopic portal imagers can produce high quality images can be acquired with a fluoroscopic EPID and a low dose. ${ }^{12,13}$ However, this type of imager is very bulky because of the mirror-lens 
system, which causes inconveniences.

\section{$\S \S$ 1.2.2 Matrix Ionization Chamber Systems}

Based on the ionization ability of $\mathrm{x}$-rays, the ionization chamber system measures the local ionization current inside a matrix ion chamber during irradiation. ${ }^{14-17}$ The electrodes of the ionizition chamber are made of two parallel circuit boards, with the sensitive volume filled with an organic fluid (Iso-octane, spectroscopical pure Merck). The chamber is covered by a $1 \mathrm{~mm}$ thick steel converter plate. High voltage and signal electrodes are etched as strips on the front and back boards of the system, respectively. The strips on one board cross those on the other perpendicularly with each crossing point acting as an ion chamber. Ions generated in the organic fluid are detected when an equilibrium is reached between ionization and recombination. The signal is readout by applying a polarizing voltage sequentially on each of the rows of electrodes, and sampling the ionization current for each column. The ion collection efficiency is about $5 \%$ due to low mobility of the ions. The spatial resolution is limited by the relatively large spacing of the electrode strips $(1.27 \mathrm{~mm})$.

\section{$\S \S$ 1.2.3 Alternative EPIDs}

With recent developments in material science and technology, new $x$-ray detectors and detection techniques have been pursued to improve electronic portal imaging in general. Antonuk et al ${ }^{18,19}$ developed a more efficient technique for detecting the fluorescent 
light produced in the fluoroscopic system. The traditional optical assembly of mirror, lens and camera was replaced with a matrix of photodiode-FET (field effect transistor) units made of hydrogenated amorphous silicon which is placed immediately below a metal plate/phosphor screen combination. Fluorescent light produced in the phosphor due to the electrons set in motion by $x$-ray radiation on the metal plate is converted with a high efficiency into electron-hole pairs in the photodiodes. These charges are then collected and stored in the capacitance of the photodiodes. After sufficient x-ray irradiation, the charges stored in the individual photodiodes are read out, one row at a time, by changing the voltage on the corresponding FET line so as to open the FET gates. This allows the signals stored in the photodiodes to propagate through the FETs onto the data lines, and then to be processed and digitized by external electronics. Compared with the traditional optical system in the fluoroscopic EPID, this hydrogenated amorphous silicon device can lead to a significant dose reduction. Placing photodiodes in close proximity to the phosphor improves light collection efficiency from less than $1 \%$ to $50 \%$. The storage ability of the diodes makes it possible to turn off the radiation during readout. Other advantages are the compactness and the inherent radiation resistance of amorphous materials.

Another approach that is of interest to portal imaging is electrostatic imaging where a uniformly charged photoconductor is exposed to x-rays transmitted through an object. The 
charge carriers stimulated by $x$-ray irradiation locally neutralize the uniformly charged photoconductor resulting in a spatially variant charge distribution. ${ }^{20}$ This charge pattern on the photoconductor, referred as the latent electrostatic image, is then made visible by a certain means. A latent electrostatic image is formed by collecting charges created by radiation in a photoconductor. Xeroradiography has also been employed by Wolfe $\mathrm{e}^{21}$ to acquired portal images in radiotherapy. Despite the high quality inherent in the latent charge image on the photoconductor, usually amorphous selenium (a-Se), the performance of xeroradiographic imagers was limited by the powder cloud development method. ${ }^{22}$ With the development of novel methods for extracting the latent image, such as photoinduced discharge with laser ${ }^{23-25}$ and the flat panel thin film transistor device ${ }^{26}$, electrostatic imaging is regaining its vitality. Recent studies have shown that electrostatic imaging by using a-Se and digital readout has various advantages over screen-film systems in mammography: higher contrast, wider dynamic range and improved quantum detective efficiency ${ }^{27,28}$. The potential of electrostatic imaging in electronic portal imaging is that charge carriers created due to irradiation by $x$-rays are collected as the output. The collection of these charge carriers is fast and efficient. It is very likely that high quality portal images can be acquired with a metal/a-Se based imager..

In view of the potential of electrostatic portal imaging with amorphous selenium to act as a charge collector for the metal plate, the Medical Physics Unit of McGill University 
developed a prototype imager consisting of a metal plate and a amorphous selenium layer. ${ }^{29}$ Part of this thesis concerns the presentation of studies concerning this prototype.

\section{$\S 1.3$ Treatment Setup and Verification}

The purpose of acquiring portal images is to verify the coverage of the target by the treatment beam. This coverage is then compared to the prescription to determine if adjustment is needed. Beam coverage evaluation requires matching a portal image to a reference image which contains the prescription. Upon decision for radiotherapy, a treatment plan is made based on diagnostic images from CT or MRI which reveal the anatomy of the patient in three dimensions. Then the patient undergoes a treatment simulation on a simulator to confirm the feasibility of the plan. A simulator differs from a therapy machine only in the $\mathrm{x}$-ray source. Instead of producing high energy photons used for therapy, it generates diagnostic $\mathrm{x}$-rays $(70 \sim 100 \mathrm{kVp})$ which deliver much less dose but produce high quality images. At each port, a simulation image is acquired. The prescribed coverage is then labelled on the simulation image as the reference of the treatment beam localization.

Traditionally, beam coverage is evaluated qualitatively by visually matching a portal film to a simulation film which leads to a high probability of localization error. The clinical impact of localization errors has been studied widely and proved to be very detrimental to the outcome of radiation treatments. In a clinical study on the treatment 
of Hodgkin's disease, Marks $e t a l^{2}$ and Kinzie $e t a l^{30}$ reported a local recurrence rate of $33 \%$ for patients with localization errors compared to that of $7 \%$ for acceptably accurate treatments. With the advent of EPIDs, the shape and location with respect to the anatomy of the radiation beam can be immediately presented after the delivery of a small radiati $\cdot \mathrm{n}$ dose. The fast image acquisition permits localization and verification images to be acquired for every beam in every treatment session with no additional material cost and only minimal extra effort. The ability of EPIDs to acquire electronic portal images much more frequently than film can further reduce the probability of setup error.

EPIDs opened the door of portal imaging for digital image processing and analysis techniques. The application of these techniques has improved the ability of EPIDs to detect patient setup errors. For example, histogram-base transformation techniques have been used to enhance the contrast of portal images for better visualization of the treatment site and the surrounding anatomy. ${ }^{31-34} \mathrm{~A}$ large amount of work has also been carried out on portal-reference image registration techniques for automatic verification of treatment setup. $^{35-45}$ Today, electronic portal imaging is also challenged by the advancement in radiation therapy. The small fields and short exposures typical of multi-beam or dynamic conformal treatments imply that few anatomical landmarks may be visible in the portal images and image registration will be a even more difficult. In order to keep up with the advancement in radiation therapy, novel techniques that can deal with difficult situations 
are being pursued. In this thesis, we will also present some of our work on automatic portal image segmentation and registration.

\section{§ 1.4 Thesis Organization}

This thesis has two major parts: (a) feasibility studies of using amorphous selenium as detector material for megavoltage portal imaging; and (b) development of software tools for portal image analysis in geometric verification of radiation treatments. We have presented results of our work in peer-reviewed journals and conferences in recent years. ${ }^{46-56}$ Part one covers Monte Carlo studies of the imaging characteristics of amorphous selenium at therapy energies including the modulation transfer function and the detective quantum efficiency (Chapter 2), and experimental studies on the sensitivity to radiation and contrast-detail performance of a prototype imager (Chapter 3).

There are two topics in the second part of this thesis. Chapter 4 addresses portal image segmentation required in beam coverage evaluation. A computer algorithm based on mathematical morphology for automatic extraction of the radiation field from a portal image will be presented. The other issue, matching radiation field to prescribed field, will be dealt with in Chapter 5. A computer tool for detecting radiation field shaping errors will also be presented. Finally, the conclusions and future work of this research will be summarized in Chapter 6. 


\section{References}

' H. F. Hare, J. Hale, and E. P. Pendergrass, "Physical and clinical aspects of supervoltage rotational therapy," Radiology 57, 157 (1951).

2 J. E. Marks, A. G. Haus, H. G. Sutton, and M. L. Griem, "Localization error in the radiotherapy of Hodgkins's disease and malignant lymphoma with extended mantle fields," Cancer 34, 83-90 (1974).

${ }^{3}$ L. E. Reinstein, H. I. Amols, P. J. Biggs, R. T. Droege, A. B. Filimonov, W. R. Lutz, and S. Shalev, AAPM Report No. 24 Radiotherapy Portal Imaging Quality(American Institute of Physics, New York, 1988).

${ }^{4}$ R. T. Droege and B. Bjarngard, "Influence of metal screens on contrast in megavoltage x-ray imaging," Medical Physics 6, 515-518 (1979).

5 P. B. Dunscombe and K. Fox, "The precision of determining compliance with prescribed fields from conventional portal films," British Journal of Radiology 62, 48-52 (1989).

${ }^{6}$ N. Baily, R. Horn, and T. Kampp, "Fluoroscopic visualization of megavoltage therapeutic X-ray beams," Int. J. Radiat. Oncol. Biol. Phys. 6, 935-939 (1980).

7 J. Leong, "Use of digital fluoroscopy as an on-line verification device in radiation therapy," Physics of Medicine and Biology 31, 985-992 (1986).

8 S. Shalev, T. Lee, K. Lesczcynski, S. Cosby, and T. Chu, "Video techniques for 
on-line portal imaging, Computerized Medical Imaging and Graphics 13, 217-226 (1989).

9 A. G. Visser, H. Huizenga, V. G. M. Althof, and B. N. Swanenburg, "Performance of a prototype fluoroscopic radiotherapy imaging system," Int. J. Radiat. Oncol. Biol. Phys. 18, 43-50 (1990).

10 P. Munro, J. A. Rawlinson, and A. Fenster, "A digital fluoroscopic imaging device for radiotherapy localization," Proceedings of SPIE 1090, 321-329 (1989).

11 A. L. Boyer, L. Antonuk, A. Fenster, M. van Herk, H. Meertens, P. Munro, L. Reinstein and J. Wong, "A review of electronic portal imaging devices(EPIDs)," Medical Physics 19, 1-16 (1992).

12 A. Ezz, P. Munro, A. T. Porter, J. Battista, D. A. Jaffray, A. Fenster, and S. Osborne, "Daily monitoring and correction of radiation field placement using a video-based portal imaging system: a pilot study," Int. J. Radiat. Oncol. Biol. Phys. 22, 159-165 (1992).

13 D. A. Jaffray, K. Chawla, C. Yu, and J. W. Wong, "Dual beam imaging for online verification of radiotherapy field placement," Int. J. Radiat. Oncol. Biol. Phys. 33, $1273-1280$ (1995).

14 M. van Herk and H. Meertens, "A digital imaging system for portal verification," in Proceedings of the 9th International Conference on the Use of Computers in Radiation 
Therapy (Elsevier North-Holland, Amsterdam 1987), 371-374.

15 M. van Herk and H. Meertens, "A matrix ionization chamber imaging device for on-line patient setup verification during radiotherapy," Radiotherapy and Oncology 11, 369-378 (1988).

16 H. Meertens, J. Bijhold, and J. Strackee, "A method for measurement of field placement errors in digital portal images," Physics in Medicine and Biology 35, 1493-1502 (1990).

17 M. van Herk, "The physics of a liquid filled ionization chamber with pulsed polarizing voltage," in Conference Records of the 10th International Conference on Conduction and Breakdown in Dielectric Liquid (IEEE, New York 1990), 126-130..

18 E. Antonuk, J. Yorkston, J. Boudry, M. Longo, and R. A. Street, "Large area amorphous silicon photodiode arrays for radiotherapy and diagnostic imaging," Nuclear Instrumentation and Method A310, 460-464 (1989).

19 E. Antonuk, J. Yorkston, J. Boudry, M. Longo, J. Jimenez, and R. A. Street, "Development of hydrogenated amorphous silicon sensors for high energy photon radiotherapy imaging," Medical Physics NS-37(2), 165-170 (1990).

20 J. W. Boag, "Xeroradiography," Physics in Medicine and Biology 18, 3-37 (1973)

21 L. Wolfe, L. Kalisher, and B. Considine, "Cobalt-60 treatment field verification by xeroradiography," American Journal of Roentgenology 118, 916-918 (1973). 
I. Brodic and R. A. Gutcheck, "Minimum exposure estimates for information recording in diagnostic radiology," Medical Physics 12, 362-367 (1985).

${ }^{23}$ A. Zermeno, T, Kirby, R. Cowart, L. Marsh, and P. Ong, "Laser readout of electrostatic images," Proceedings of SPIE 173, 81-87 (1979).

24 E. L. Couk, J. D. Edwards, O. L. Nelson and J. E. Potts, "Performance of a high resolution radiographic detector," in The society of Imaging Science and Techmologv 47th Annual Conference ICPS, 669 (1994).

25 J. A. Rowlands and D. M. Hunter, "X-ray imaging using amorphous selenium: Photoinduced discharge (PID) readout for digital general radiography," Medical Physics 22, 1983-2005 (1995).

26 W. Zhao and J. A. Rowlands, "X-ray imaging using amorphous selenium: Feasibility of a flat panel self-scanned detector for digital radiology," Medical Physics 22 , 1595-1604 (1995).

27 I. Brodie and R. A. Gutcheck, "Radiographic information theory and application to mammography," Medical Physics 9, 79-94 (1982).

28 U. Neitzel, I. Maack, and S. Gunther-Kohfahl, "Image quality of a digital chest radiography system based on a selenium detector," Medical Physics 21, 509-516 (1994).

29 B. G. Fallone and T. Falco, "Megavoltage imaging method using a combination of 
a photoreceptor with a high energy photon converter and intensifier," International Patent File (1996).

30 J. J. Kinzie, G. E. Hanks, C. J. Maclean, and S. Kramer, "Patterns of care study: Hodgkin's disease relapse rates and adequacy of portals," Cancer 52, 2223-2226 (1983).

31 S. M. Pizer, E. P. Amburn, J. D. Austin, R. Cromartie, A. Geselowitz, T. Greer. B. H. Romeny, J. B. Zimmerman, and K. Zuiderveld, "Adaptive histogram equalization and its variations," Computer Vision, Graphics and Image Processing 39, 355-368 (1987).

32 K. W. Leszczynski and S. Shalev, “A robust algorithm for contrast enhancement by local histogram modification," Imag. Vision Comp. 7 205-209 (1989).

33 I. Crooks and B. G. Fallone, "Contrast enhancement of portal images by selective histogram equalization," Medical Physics 20, 199-204(1993).

34 Y. Bao, "A novel histogram modification approach for medical image enhancement," SPIE 2167, 755-765 (1994).

35 S. M. Jones and A. L. Boyer, "Investigation of a FFT-based correlation technique for verification of radiation treatment setup," Medical Physics 18, 1116-1125 (1991).

36 J. M. Balter, C. A. Pelizzari, and G. T. Y. Chen, "Correlation of projection radiographs in radiation therapy using open curve segments and points," Medical Physics 
19, 329-334 (1992).

37 K. W. Leszczynski, S. Shalev, and N. S. Cosby, "The enhancement of radiotherapy verification images by an automated edge detection technique," Medical Physics 19 $611-621$ (1992).

38 K. Leszczynski, S. Shalev, and G. Gluhchev, "Verification of radiotherapy treatments: Computerized analysis of the size and shape of radiation fields," Medical Physics 20, 687-694 (1993).

39 G. X. Ding, S. Shalev and G. Gluchev, "A $\rho-\theta$ technique for treatment verification in radiotherapy and its clinical applications," Medical Physics 20, 1135-1143 (1993).

40 J. Moseley and P. Munro, "A semiautomatic method of registration of portal images," Medical Physics 21, 551-558 (1994).

41 T. Radcliffe, R. Rajapakshe, and S. Shalev, "Pseudosorrelation: A fast, robust, absolute, grey-level image alignment algorithm," Medical Physics 21, 761-679 (1994).

42 M. van Herk, K. Gilhuijs, P. Williams, P. Cinquin, L. Cionini, and W. Swindel, "Role of electronic portal imaging in high dose/high precision radiotherapy," Radiotherapy and Oncology 21, 269-270 (1994).

43 D. S. Fritsch, E. L. Chaney, A. Boxwala, M. J. McAuliffe, S. Raghavan, A. Thall, and J. R. D. Earnhart, "Core-based portal image registration for automatic radiotherapy 
treatment verification," Int. J. Radiat. Oncol. 29, 1287-1300 (1995).

44 K. Leszczynski and S. Loose and P. Dunscombe, "Segmented chamfer matching for prescription-treatment image registration in radiotherapy," Physics in Medicine and Biology 40, 83-94 (1995) .

45 D. H. Hristov and B. G. Fallone, "A grey-level image alignment algorithm for registration of portal images and digitally reconstructed radiographs," Medical Physics 23, 75-84 (1996).

$46 \mathrm{H}$. Wang and B. G. Fallone, "A robust algorithm for automatic segmentation and correlation of portal images," in Proceedings of the Joint Conference of The Canadian Organization of Medical Physicists and The Canadian Medical and Biological Engineering Society (Ottawa, Ontario, 1993), 44-45.

47 H. Wang and B. G. Fallone, "A robust morphological algorithm for automatic radiation field-extraction and correlation of portal images," Medical Physics 21, 237-244 (1994).

48 H. Wang and B. G. Fallone, "A sequential minimization technique in chamfer matching," in Proceedings of 3rd International Workshop On Electronic Portal Imaging (San Francisco, California 1994).

49 H. Wang and B. G. Fallone, "A model-based algorithm for accurate extraction of the radiation field from double-exposure portal images," in Proceedings of 3rd 
International Workshop On Electronic Portal Imaging (San Francisco, California 1994).

$50 \mathrm{H}$. Wang and B. G. Fallone, "A mathematical model of radiation field edge localization," Medical Physics 22, 1107-1110 (1995).

51 H. Wang and B. G. Fallone, "Matching treatment and prescription fields with a combined moment-chamfer matching technique," in Proceedings of 41 st Annual Meeting Canadian Organization of Medical Physicists \& Canadian College of Physicists in Medicine with the Canadian Association of Radiologists, (Montreal, Quebec 1995).

52 H. Wang and B. G. Fallone, "Model based localization of the radiation field border in double-exposure portal images," in Proceedings of 4lst Annual Meeting Canadian Organization of Medical Physicists \& Canadian College of Physicists in Medicine with the Canadian Association of Radiologists (Montreal, Quebec, 1995).

53 H. Wang and B. G. Fallone, "A simplified technique for matching radiation and prescribed field," Radiology and Oncology 30, 58-65 (1996).

54 H. Wang and B. G. Fallone, "Monte Carlo calculations of imaging characteristics of a-Se in electrostatic portal imaging," in Proceedings of the 42nd Annual Meeting of Canadian Organization of Medical Physicists (Vancouver, British Colombia, 1996), 286-289.

${ }^{55}$ H. Wang and B. G. Fallone, "A metal screen-amorphous selenium based image 
receptor in megavoltage portal imaging (Abstract)," Medical Physics 23, 1130 (1996).

56 B. G. Fallone, T. Falco, H. Wang, and N. Araj, "An electrostatic-based detector for portal imaging," in The 4th International Workshop on Electronic Portal Imaging: Portal Imaging in Theory and Practice (Amsterdam, Holland ,1996). 


\section{Chapter 2}

\section{Imaging Characteristics of Metal/a-Se: Monte Carlo Studies}

$\S 2.1 \quad$ Introduction $\ldots \ldots \ldots \ldots \ldots \ldots \ldots \ldots \ldots \ldots \ldots$

$\S 2.2$ Amorphous Selenium (a-Se) for Portal Imaging $\ldots \ldots \ldots \ldots$

$\S 2.3$ Receptor Blur and Modulation Transfer Function $\ldots \ldots \ldots \ldots$

$\S 2.4$ Quantum Noise and Detective Quantum Efficiency . . . . . . . 27

$\S 2.5$ Monte Carlo Simulations of Energy Deposition . . . . . . . . 30

$\S \S 2.5 .1 \quad$ The EGS4 Code . . . . . . . . . . . . . . 30

$\S \S 2.5 .2 \quad$ Receptor Geometry . . . . . . . . . . . . . . 31

$\S \S 2.5 .3 \quad$ Calculations of MTF $\ldots \ldots \ldots \ldots \ldots \ldots \ldots \ldots$

$\S \S 2.5 .4$ Calculations of Absorption Efficiency and DQE $\ldots \ldots \ldots 33$

$\S 2.6 \quad$ Resuits . . . . . . . . . . . . . . . . . 34

$\S \S 2.6 .1 \quad$ Modulation Transfer Function . . . . . . . . . . . . 34

$\S \S 2.6 .2$ Quantum Absorption Efficiency . . . . . . . . . .46

$\S \S 2.6 .3$ Detector Response $\ldots \ldots \ldots$. . . . . . . . . 47

$\S \S 2.6 .4$ Statistical Factor $\ldots \ldots \ldots \ldots \ldots \ldots \ldots$

$\S \S 2.6 .5$ Detective Quantum Efficiency . . . . . . . . . . 51

$\S 2.7$ Conclusions $\ldots \ldots \ldots \ldots \ldots \ldots \ldots \ldots \ldots \ldots \ldots \ldots$ 


\section{§ 2.1 Introduction}

Image acquisition in transmission radiology starts with the detection of $\mathrm{x}$-rays transmitted through a patient. The change of some physical parameter caused by the interaction between the $\mathrm{x}$-rays and the detector is then extracted as the output signal by a certain means. Despite the difference in the type of the output signal, any image receptor is basically an energy detector in the first stage of the image formation process where the intensity of the input is measured by the amount of energy deposited in the detector. Upon interaction with the detector, a photon that traversed through the patient is either absorbed or scattered. Except for coherent scattering, the energy of the photon is completely or partially transferred to an electron set in motion or to a positron-electron pair generated. Interaction with the detector causes the secondary charged particles to lose energy, slow down and eventually stop. The scattered photon can go on and interact with the detector again until it is absorbed. This multiple interaction mechanism is called coupled photon-electron transport. As a result, an incident photon generates a photonelectron "shower" in the detector introducing an uncertainty in the spatial location of the incident point. This lateral spread in the output is called receptor blur. Due to the stochastic nature of the coupled photon-electron transport, the amount of energy deposited by an incident photon in the detector is random. This randomness in energy deposition introduces a fluctuation in the output signal known as quantum noise. ${ }^{1-3}$ Originated from 
the interaction of $\mathrm{x}$-rays with the receptor, the magnitude of receptor blur and quantum noise depend on the energy of the $x$-rays and the composition and geometry of the receptor. The objective of this chapter is to investigate this dependence in order to optimize an amorphous-selenium-based receptor for portal imaging.

\section{§ 2.2 Amorphous Selenium (a-Se) for Portal Imaging}

A latent image on the a-Se surface is formed via local neutralization of the uniform charge distribution achieved through some charging procedure before irradiation. The extent of this local neutralization is proportional to the number of electron-hole pairs generated by the radiation in a small volume which is proportional to the energy deposited in the volume. Conventionally, a layer of selenium is deposited on a metal substrate. The selenium is directly exposed to $\mathrm{x}$-rays transmitted through a patient. This receptor configuration is used in diagnostic imaging where the beam energy is low.

In portal imaging, an image is acquired with a therapy beam with high penetrating ability which reduces detection efficiency. A metal plate is combined with a portal image receptor. For example, a portal film is placed in a cassette with a copper plate on the beam entrance side. Because of the high attenuation coefficient of the metal, a significant portion of the incident photon beam is converted to secondary electrons. It is the interaction of the electrons with the receptor that is responsible for image formation. 
Metal plates are also employed in fluoroscopic EPIDs and matrix ion chamber EPIDs to enhance detector response.

Introducing a metal plate will not only increase detector efficiency but will also affect the noise level. Droege and Bjarngard ${ }^{4}$ reported that a metal plate can significantly reduce the scatter to primary ratio when used with portal films. Jaffray $e t a l^{5}$ reported that a copper plate can reduce quantum noise associated with $\mathrm{x}$-ray absorption in phosphor screens thus improve the detective quantum efficiency.

An amorphous selenium portal imaging system has been under development in the Medical Physics Unit at McGill University. Fallone $e a^{6,7}$ proposed that the metal substrate of a selenium receptor be used as the front plate so to serve as a conversion medium. However, the substrate has to be customized for this new purpose. In this chapter, we will calculate the modulation transfer function and the detective quantum efficiency of a metal-amorphous selenium image receptor at different spatial frequencies to reveal the effect of a front metal plate on detector sensitivity, noise level and spatial resolution of amorphous selenium detectors.

\section{§ 2.3 Receptor Blur and Modulation Transfer Function}

The most straightforward mathematical representation of receptor blur is the point spread function. Given an impulse input $\delta(x, y)$, the output $p(x, y)$ is defined as the point spread function of the receptor. Receptor blurring alters the one to one 
correspondence between an object point and its image. Consider an impulse input $I\left(x^{\prime}, y^{\prime}\right) \delta\left(x-x^{\prime}, y-y^{\prime}\right)$ at $\left(x^{\prime}, y^{\prime}\right)$ where $I\left(x^{\prime}, y^{\prime}\right)$ is the magnitude of the impulse. It will have a contribution $I\left(x^{\prime}, y^{\prime}\right) p\left(x-x^{\prime}, y-y^{\prime}\right)$ to the output at $(x, y)$. The output $O(x, y)$ at $(x, y)$ is the sum of the contributions from all points

$$
O(x, y)=\int_{-\infty}^{\infty} \int_{-\infty}^{\infty} I\left(x^{\prime}, y^{\prime}\right) p\left(x-x^{\prime}, y-y^{\prime}\right) d x^{\prime} d y^{\prime}
$$

This means that a point on the image is not only related to the corresponding point on the object but also to all the other points. Receptor blur causes loss of image detail, and the receptor does not transfer spatial information equally at every detail level. This can be viewed from another perspective by applying the Fourier transform to Eq. (2.1)

$$
\mathcal{O}(u, v)=\mathcal{S}(u, v) \cdot \mathcal{I}(u, v)
$$

where

$$
\mathcal{I}(u, v)=\int_{-\infty}^{\infty} \int_{-\infty}^{\infty} I(x, y) \operatorname{cxp}[-2 \pi i(u x+v y)] d x d y
$$

and

$$
\mathcal{O}(u, v)=\int_{-\infty}^{\infty} \int_{-\infty}^{\infty} O(x, y) \exp [-2 \pi i(u x+v y)] d x d y
$$

are the components of the input and output at spatial frequency $(u, v)$, respectively, and the Fourier transform of the point spread function

$$
\mathcal{S}(u, v)=\int_{-\infty}^{\infty} \int_{-\infty}^{\infty} p(x, y) \exp [-2 \pi i(u x+v y)] d x d y
$$


is the system response of the receptor. Eq. (2.2) shows that the output is modulated by the system response. For a perfect receptor which has no blur, i.e.,

$$
p(x, y)=\delta(x, y)
$$

where $\delta(x, y)$ is the Dirac delta function in two dimension, the system response is constant at all spatial frequencies, i.e.,

$$
S(u, v)=\int_{-\infty}^{\infty} \int_{-\infty}^{\infty} \delta(x, y) \exp [-2 \pi i(u x+v y)] d x d y=1
$$

implying that the receptor can pass on all the information from the input to the output. For a practical receptor, the system response decreases as the spatial frequency increases because the point spread function is not a delta function. The property of a receptor can be characterized by the system response or the point spread function. However, measuring the point spread function is very difficult. Image blur and signal transfer are usually dealt with in terms of the line spread function and the modulation transfer function.

Defined as a line integral of the point spread function

$$
l(x)=\int_{-\infty}^{\infty} p(x, y) d y
$$

the line spread function $l(x)$ specifies image blur in one dimension. It can be measured by scanning the image of a very narrow slit. The modulation transfer function is defined 
as the modulus of the Fourier transform of the line spread function

$$
\begin{aligned}
\operatorname{MTF}(u) & =\left|\int_{-\infty}^{\infty} l(x) \exp (-2 \pi i u) d x\right| \\
& =\left|\int_{-\infty}^{\infty} \int_{-\infty}^{\infty} p(x, y) \exp (-2 \pi i u) d x d y\right| \\
& =|\mathcal{S}(u, 0)|
\end{aligned}
$$

and can be proved to be the profile of the system response on the $u$ axis.

The measurement of the line spread function $l(x)$ can be modelled as a smoothing process followed by a sampling process. Mathematically, the measured line spread function $l_{m}(x)$ can be expressed as

$$
l_{m}(x)=\left[l(x) \otimes \operatorname{rect}\left(\frac{x}{a}\right)\right] \cdot \operatorname{comb}\left(\frac{x}{b}\right)
$$

where the convolution with

$$
\operatorname{rect}\left(\frac{x}{a}\right)=\left\{\begin{array}{ll}
1, & \mid x \leq \frac{a}{2} \\
0, & \mid x>\frac{a}{2}
\end{array} \mid\right.
$$

represents the averaging effect of the aperture size $a$, and the multiplication with

$$
\operatorname{comb}\left(\frac{x}{b}\right)=\sum_{n=-\infty}^{n=+\infty} \delta(x-n b)
$$

represents the sampling with a spacing of $b$. The upper limit of the spatial frequency at which the modulation transfer function can be measured is determined by the Nyquist criterion:

$$
u_{\max }=\frac{1}{2 b}
$$


The measured modulation transfer function $M T F_{m}(u)$ is then given by the modulus of the Fourier transform of $l_{m}(x)$ :

$$
\begin{aligned}
\operatorname{MTF}_{m}(u) & =\left|\mathcal{F}\left\{l_{m}(x)\right\}\right| \\
& =|[M T F(u) \cdot \operatorname{sinc}(a u)] \otimes \operatorname{comb}(b u)|
\end{aligned}
$$

which contains a truncation error introduced by the multiplication with $\operatorname{sinc}(a u)$ and aliasing artifact introduced by the convolution with $\operatorname{comb}(b u)$. Precautions have to be taken in the selection of the aperture size and sampling rate in order to keep systematic errors under an acceptable limit. The convolution in Eq. (2.14) causes overlapping of adjacent cycles. This overlap can be reduced by increasing the aperture size $a$. A larger aperture reduces the amplitudes of the sidelobes of $\operatorname{sinc}(a u)$ but at the same time increases the truncation error. As a trade-off of aliasing reduction, the measured modulation transfer function will deviate more from the true value. The current convention used in modulation transfer function measurements is $a=2 b$, i.e., the aperture should be at least twice the size of the sampling interval. This convention ensures a less than $2 \%$ systematic error in the sampled data. ${ }^{8}$

\section{§ 2.4 Quantum Noise and Detective Quantum Efficiency}

Quantum noise in X-ray imaging originates from the fluctuation of the incident photon flux characterized by Poisson statistics and the randomness of the amount of energy deposited by each $\mathrm{x}$-ray photon in the receptor. While the former determines the noise level of the input, the latter is the reason for the degradation of the signal to noise level 
introduced by the receptor. This degradation is usually characterized by the detective quantum efficiency defined as:

$$
D Q E=\left(\frac{S N R_{\text {out }}}{S N R_{\text {in }}}\right)^{2}
$$

For an amorphous selenium receptor, the energy deposited by an incident photon is used to create electron-hole pairs which are responsible for the formation of the electrostatic image. The number of these charge carriers produced by $N$ incident photons of energy $E_{\text {in }}$ is given by

$$
\frac{\int_{0}^{E_{i n}} n\left(E, E_{i n}\right) E d E}{W},
$$

where $n\left(E, E_{i n}\right)$ is the average number of photons that deposited the amount of energy $E$, and $W$ is the average energy required to generate one electron-hole pair. The fluctuation of $n\left(E, E_{i n}\right)$ is $\sqrt{n\left(E, E_{i n}\right)}$. Considering the absorbed energy distribution, the total uncertainty is:

$$
\frac{\sqrt{\int_{0}^{E_{1 n}}\left(\sqrt{n\left(E, E_{i n}\right)} E\right)^{2} d E}}{W} .
$$

Therefore,

$$
S N R_{\text {out }}=\frac{\int_{0}^{E_{1 n}} n(E) E d E}{\sqrt{\int_{0}^{E_{1 n}} n(E) E^{2} d E}}
$$




$$
S N R_{i n}=\frac{N}{\sqrt{N}}
$$

and

$$
\begin{aligned}
D Q E & =\left(\frac{\int_{0}^{E_{i n}} \frac{n\left(E, E_{i n}\right)}{N} E d E}{\sqrt{\int_{0}^{E_{i n}} \frac{n\left(E, E_{i n}\right)}{N} E^{2} d E}}\right)^{2} \\
& =\frac{M_{1}^{2}\left(E_{i n}\right)}{M_{2}\left(E_{i n}\right)}
\end{aligned}
$$

where

$$
M_{i}\left(E_{i n}\right)=\int_{0}^{E_{i n}} \frac{n\left(E, E_{i n}\right)}{N} E^{i} d E
$$

is the $i$ th moment of the normalized pulse height spectrum $\frac{n\left(E, E_{\text {in }}\right)}{N}$ from incident photons of energy $E_{i n}$. Equation 2.21 is the $\mathrm{DQE}$ at zero spatial frequency because spatial information transfer is not considered. DQE at a non zero spatial frequency is lower as the receptor can not fully transfer the information at that detail level. DQE as a function of spatial frequency can be expressed as:

$$
D Q E(f)=D Q E(0) \cdot M T F^{2}(f)
$$

provided that quantum noise is white noise. This is justifiable since the input noise is determined by the Poisson statistics and the output noise is determined by the fluctuation in the energy deposited by a photon. Neither of them depends on the spatial frequency of the input under the assumption that $\mathrm{x}$-rays are photons and the detector is a large continuum. 


\section{§ 2.5 Monte Carlo Simulations of Energy Deposition}

\section{$\S \S$ 2.5.1 The EGS4 Code}

The coupled photon-electron transport within the detecior was simulated with the Electron Gamma Shower (EGS4) $\operatorname{code}^{9}$ which has been extensively used for radiation dose calculation in the energy range from 1 to $10 \mathrm{MeV}$ and has been proven to produce reliable results. As a general purpose software package, EGS4 consists of two major parts: the system code that handles the physics of the coupled photon-electron transport and the user code that defines the geometry and type of the medium/media. The user code also specifies which physical observable(s) will be scored. In our simulations, we used the EGS4 package distributed by the National Research Council of Canada. In addition to the system code, this package also provides two general purpose programs, XYZDOS and DOSRZ, that allow the user to define the simulation geometry in Cartesian and polar coordinate systems. These two programs include the Parameter Reduced Electron Step Transport Algorithm (PRESTA) which can reduce the dependence of charged particle transport on user-selected parameters. ${ }^{10}$ For simplicity, we used the default values of the parameters for the PRESTA algorithm. Density effect corrections were also included in the collisional stopping powers. The $\mathrm{K}$ fluorescence production was not considered since it is not significant in the megavoltage energy range. ${ }^{5}$ The parameters controlling the transport were set as the following: $E C U T=A E=0.521 \mathrm{MeV}$, 
$\mathrm{PCUT}=\mathrm{AP}=0.01 \mathrm{MeV}$, where ECUT is the minimum total energy of electrons that are transported, PCUT is the minimum total energy of photons that are transported, AE and AP are the energy thresholds for creation of secondary electrons and photons, respectively. Since the spectrum of the beam transmitted through the patient is not known precisely, monoenergetic photons $(0.1 \sim 6 \mathrm{MeV})$ were used in all simulations. Test runs of each type of simulation were performed to verify the consistency of the result before the simulations used for calculating the imaging characteristics of the metal/a-Se receptor. The results of simulation runs are consistently well within $1 \%$ of each other.

\section{$\S \S 2.5 .2$ Receptor Geometry}

The simulations were run on four receptors that were used in the experimental studies which will be presented in the next chapter. As illustrated in Figure 2.1 , a layer of amorphous selenium is coated on an $8 \times 8 \mathrm{in}^{2}$ front metal plate. As a build-up material, the metal plate converts the incident photons into electrons. Intuitively, the optimal thickness of the metal plate should be the depth $d_{\max }$ where electronic equilibrium is reached. This depth represents the point at which the energy deposition is at its maximum. Beyond this ciepth, energy absorption decreases because the primary photon beam is attenuated and electrons do not travel over a certain range. However, fabrication of a-Se receptors is complicated and our choice is limited by availability. Three of the four receptors (Noranda Advanced Materials Inc., Pointe Claire, QC) have a $2 \mathrm{~mm}$ thick 
narrow parallel beam of monoenergetic photons

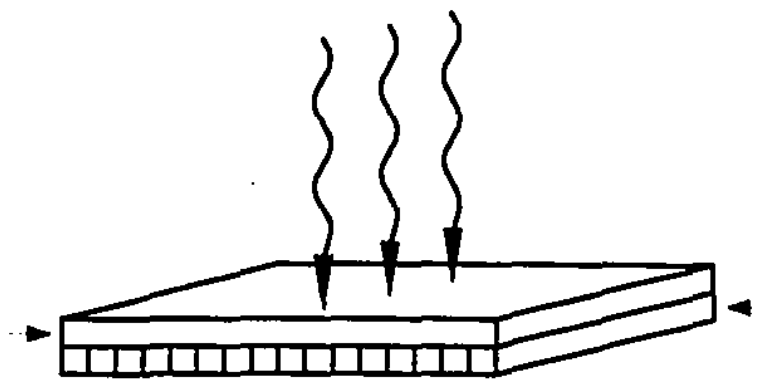

metal plate

a-Se

Figure 2.1. Geometry of the line spread function simulation

aluminum plate with different thicknesses of a-Se: $150 \mu \mathrm{m}, 300 \mu \mathrm{m}$ and $500 \mu \mathrm{m}$. The other receptor consists of a $1 \mathrm{~mm}$ copper plate and a $300 \mu \mathrm{m}$ thick layer of a-Se.

\section{$\S \S$ 2.5.3 Calculations of MTF}

Figure 2.1 shows the simulation of the line spread function which was run with the user code XYZDOS. A $2 \mu \mathrm{m} \times 20 \mathrm{~cm}$ parallel beam of monoenergetic photons is incident at the center of a $20 \times 20 \mathrm{~cm}^{2}$ receptor. The a-Se layer of the receptor is divided into a series of $5 \mu \mathrm{m}$ wide strips inside which the deposited energies are scored. Every two adjacent points are then averaged:

$$
\begin{aligned}
& l_{m}\left(x_{i}\right)=\frac{1}{2}\left[E\left(x_{i}\right)+E\left(x_{i+1}\right)\right] \\
& x_{i}=(i-1) h, \quad i=1,2,3, \cdots, N
\end{aligned}
$$

to satisfy the requirement of the adequate aperture size. According to the Nyquist criterion, the sampling rate gives a cutoff frequency of $100 \mathrm{~mm}^{-1}$. The selection of 
the bin width must also ensure that multiple scattering can be modelled accurately by the EGS4 Monte Carlo code. The rule of thumb to estimate the number of multiple scattering events is

$$
N_{m s}=\operatorname{density}\left(\mathrm{g} / \mathrm{cm}^{3}\right) \cdot(Z / 8)^{\frac{1}{3}} \cdot \text { stepsize }(\mu \mathrm{m}),
$$

where $N_{m s}$ is the number of multiple scattering events, $Z$ is the atomic niיnber of the material considered. For a $5 \mu \mathrm{m}$ step size in amorphous selenium, $N_{m s}$ is approximately 35 which is sufficient. The MTF is obtained by applying the Fast Fourier Transform (FFT) to the discrete Line Spread Function. To ensure the accuracy of the results, 30 million photons were used in each simulation resulting in a statistical uncertainty less than $5 \%$ in each strip. This requires calculation times ranging from 7 to 24 hours on an SGI workstation (IRIS INDIGO, Silicon Graphics, Mountainview, CA).

\section{§§ 2.5.4 Calculations of Absorption Efficiency and DQE}

In order to calculate the absorption efficiency and the detective quantum efficiency, the energy absorbed in the entire sensitive volume and its pulse height spectrum need to be scored. Unfortunately, XYZDOS does not include the option of pulse height spectrum. The simulations had to be run with the more versatile and more user friendly DOSRZ. As shown in Figure 2.2, a pencil beam of monoenergetic photons is incident at the center of the circular detector with a radius of $10 \mathrm{~cm}$. The effect of the detector shape is negligible since the radius is sufficiently large for a pencil beam. Equal energy bin width 


\section{pencil beam of monoenergetic photons}

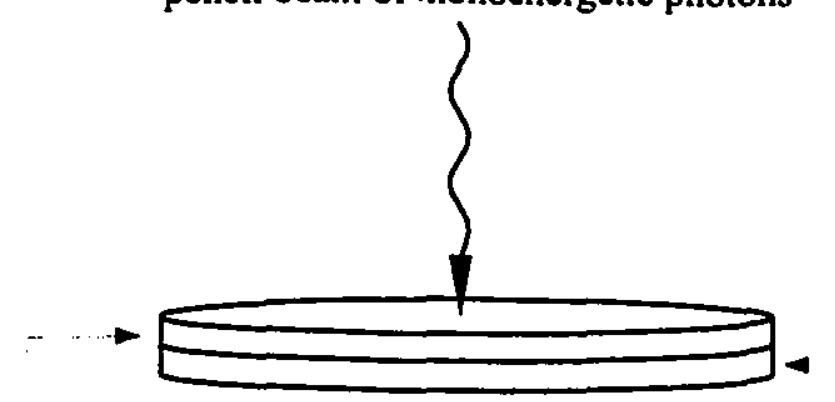

metal plate

a-Se

Figure 2.2. Geometry of the energy deposition simulation

was used in the pulse height spectrum: $0.01 \mathrm{MeV}$ for incident photons of energy less than $3 \mathrm{MeV}$ and $0.03 \mathrm{Mev}$ for $3 \mathrm{MeV}$ and above. The simulations were terminated only when the uncertainty in the pulse height spectrum became less than $10 \%$ in each bin. Approximately 72 hours were required for each run.

\section{§ 2.6 Results}

\section{§§ 2.6.1 Modulation Transfer Function}

Simulations were run for the four receptors that will be investigated experimentally in the next chapter. Three of the four receptors have a common front metal plate $(2 \mathrm{~mm}$ Al) but a different a-Se layer (150 $\mu \mathrm{m}, 300 \mu \mathrm{m}$ and $500 \mu \mathrm{m}$ thick) while the other has 


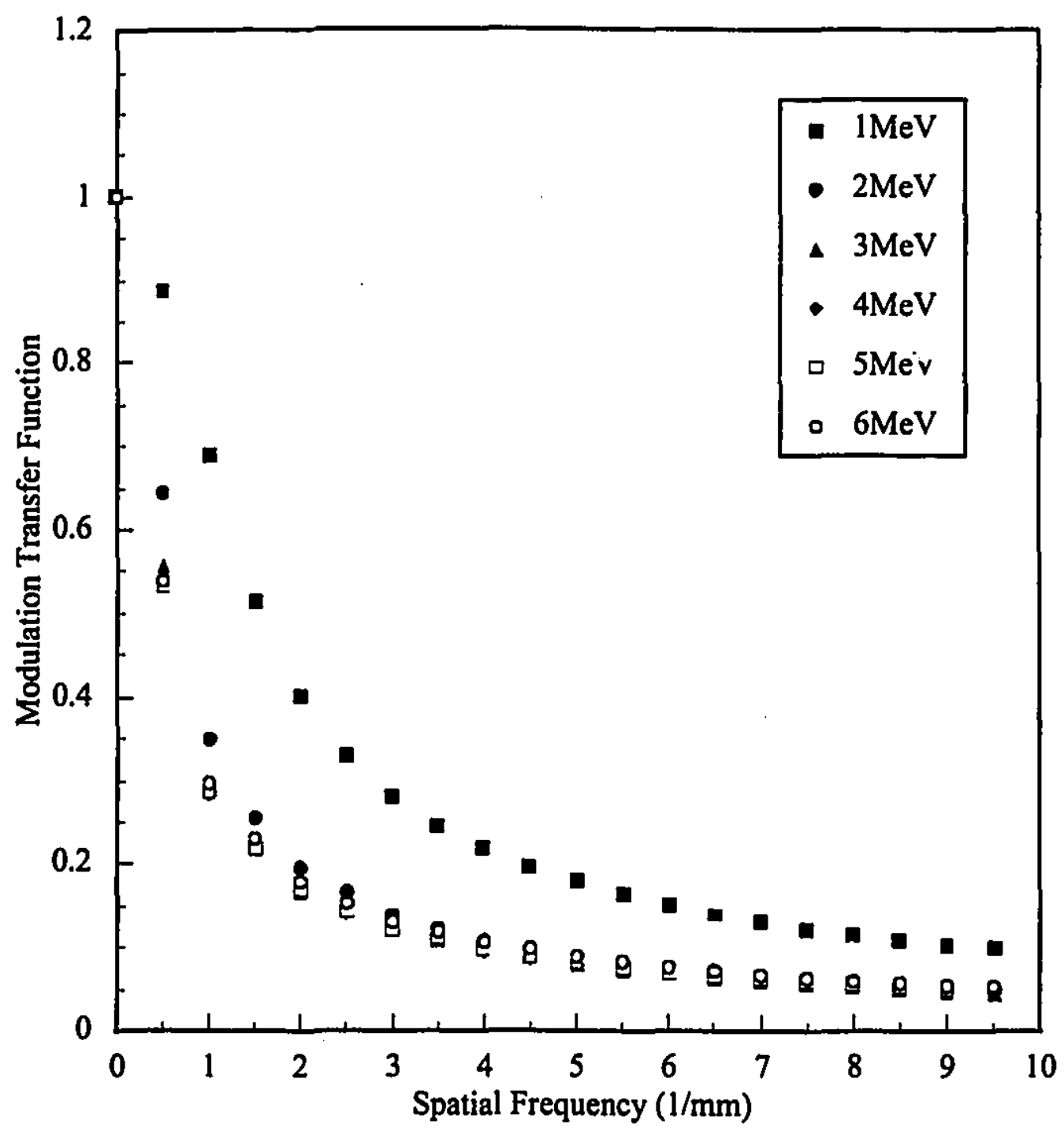

Figure 2.3. The modulation transfer functions of a $2 \mathrm{~mm} \mathrm{Al} / 0.15 \mathrm{~mm}$ a-Se receptor at various photon energies. 


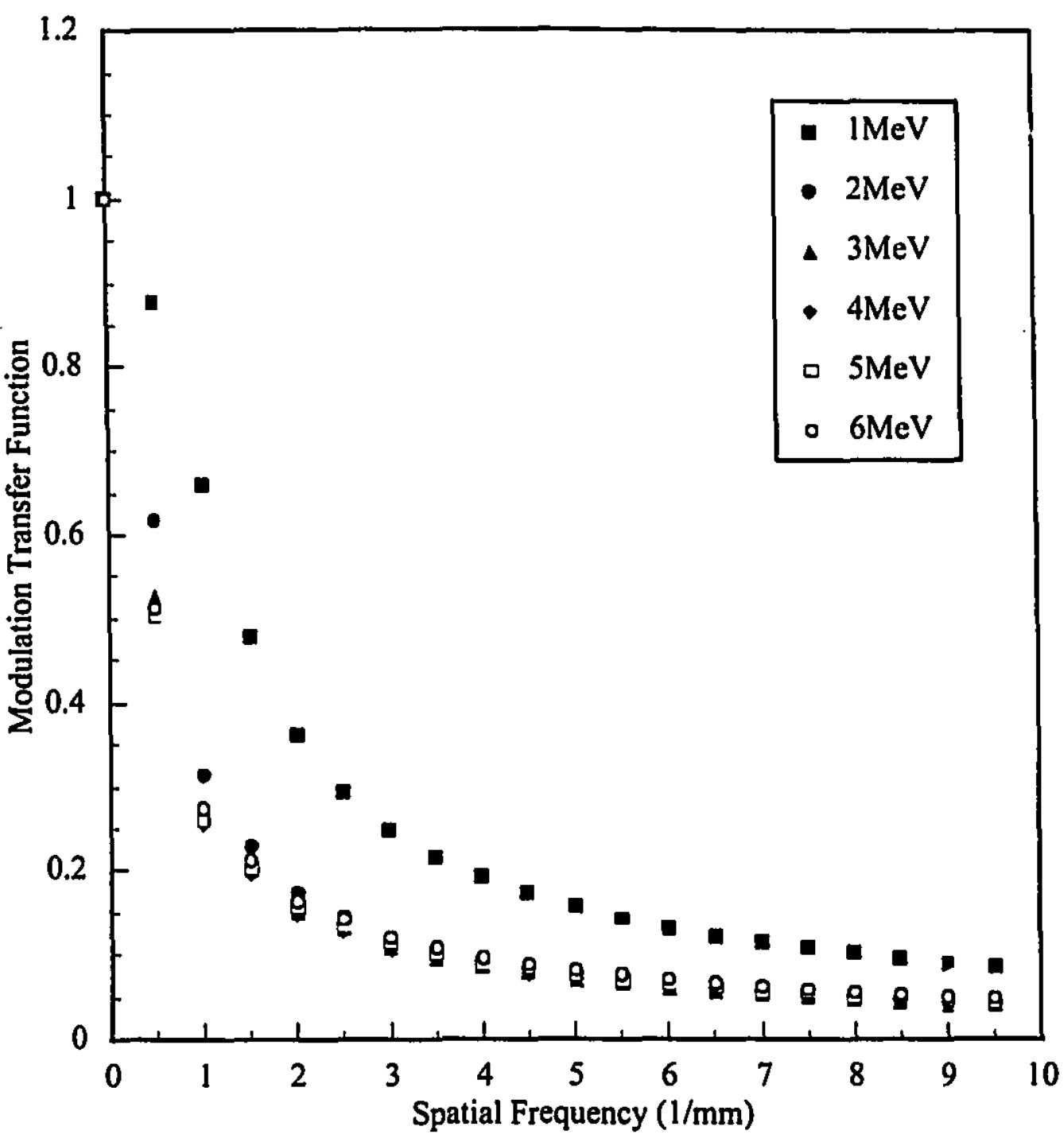

Figure 2.4. The modulation transfer functions of a $2 \mathrm{~mm} \mathrm{Al} / 0.3 \mathrm{~mm}$ a-Se receptor at various photon energies. 


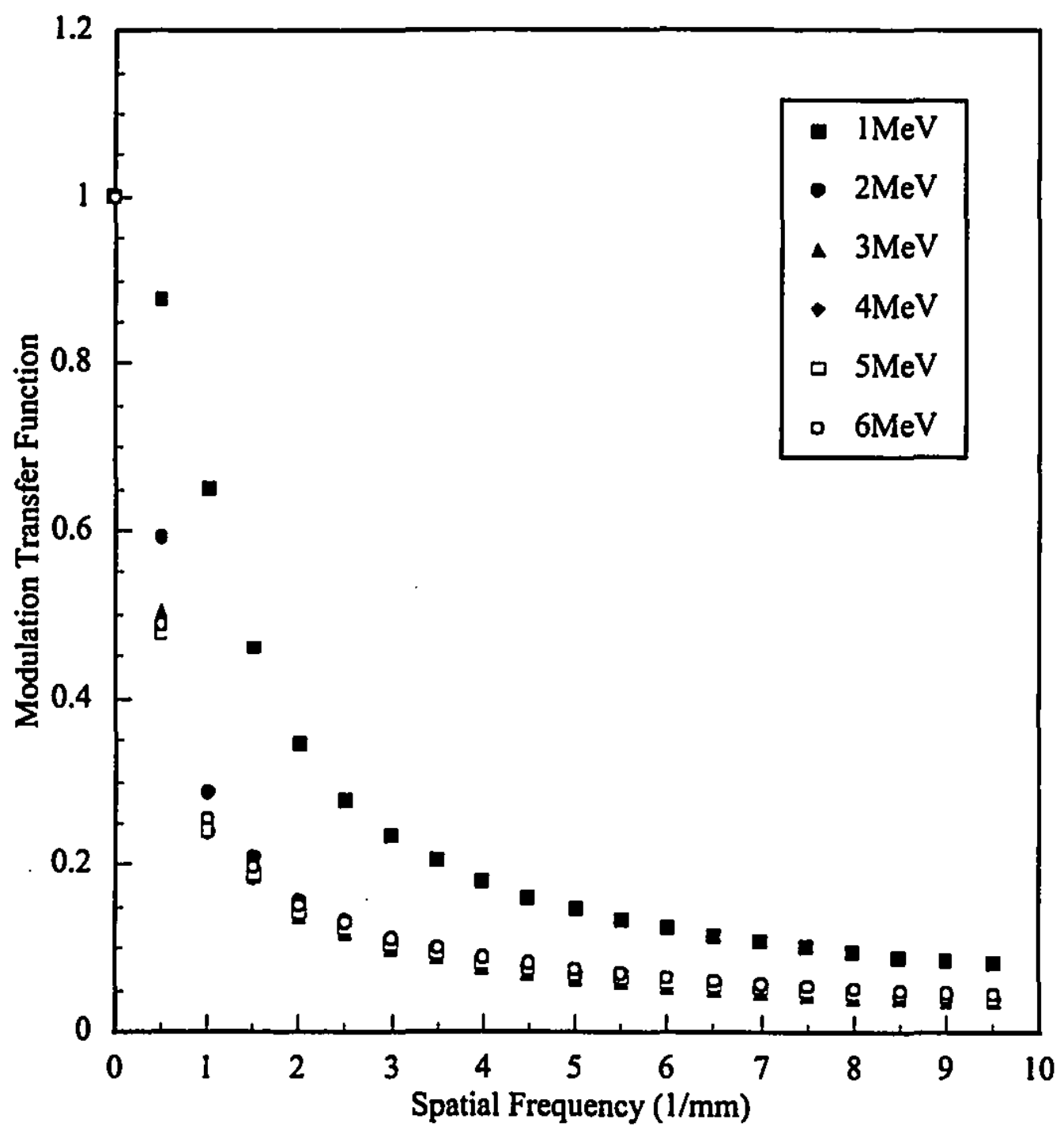

Figure 2.5. The modulation transfer functions of a $2 \mathrm{~mm} \mathrm{Al} / 0.5 \mathrm{~mm}$ a-Se receptor at various photon energies. 


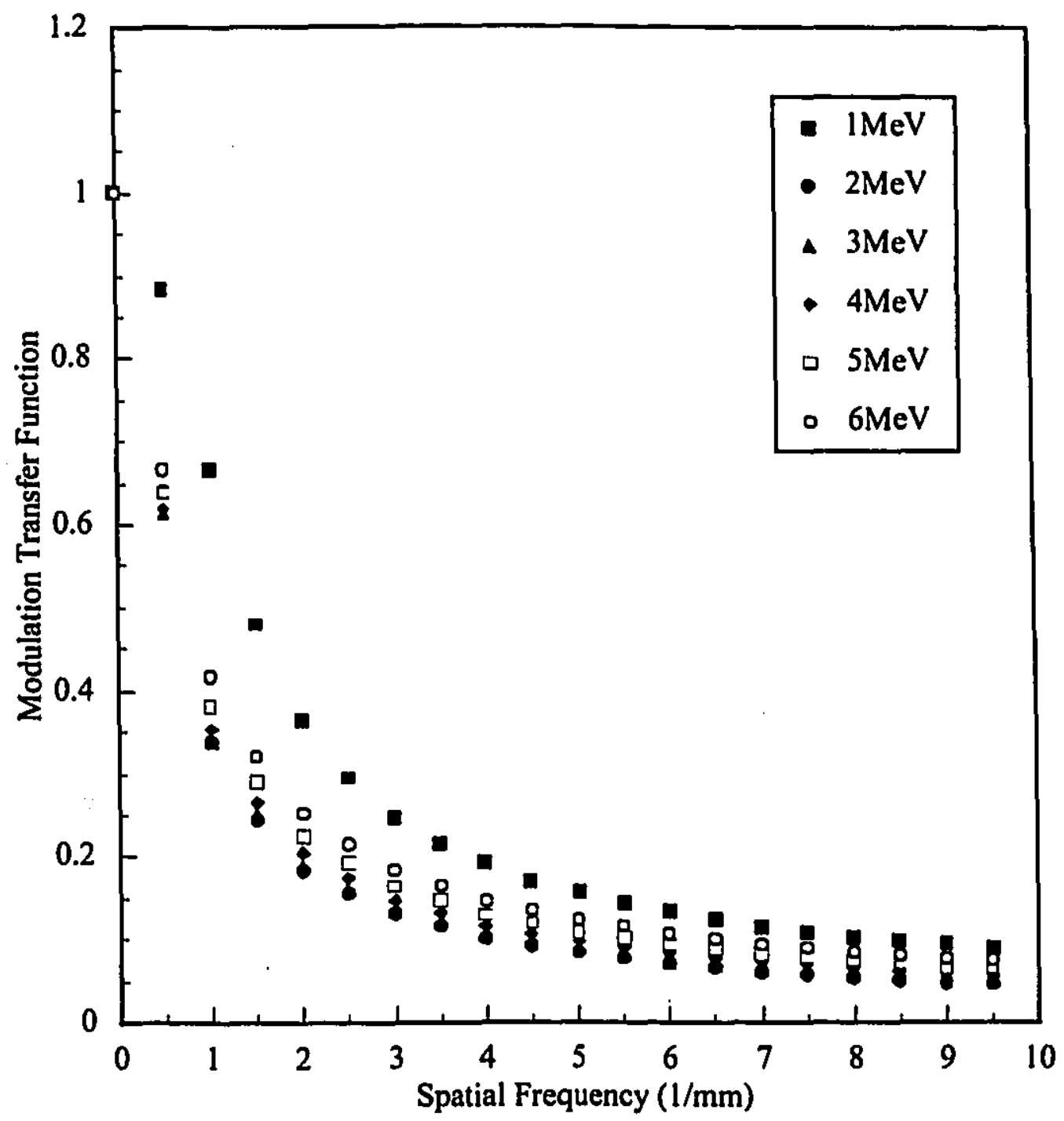

Figure 2.6. The modulation transfer functions of a $1 \mathrm{~mm} \mathrm{Cu} / 0.3 \mathrm{~mm}$ a-Se receptor at various photon energies. 


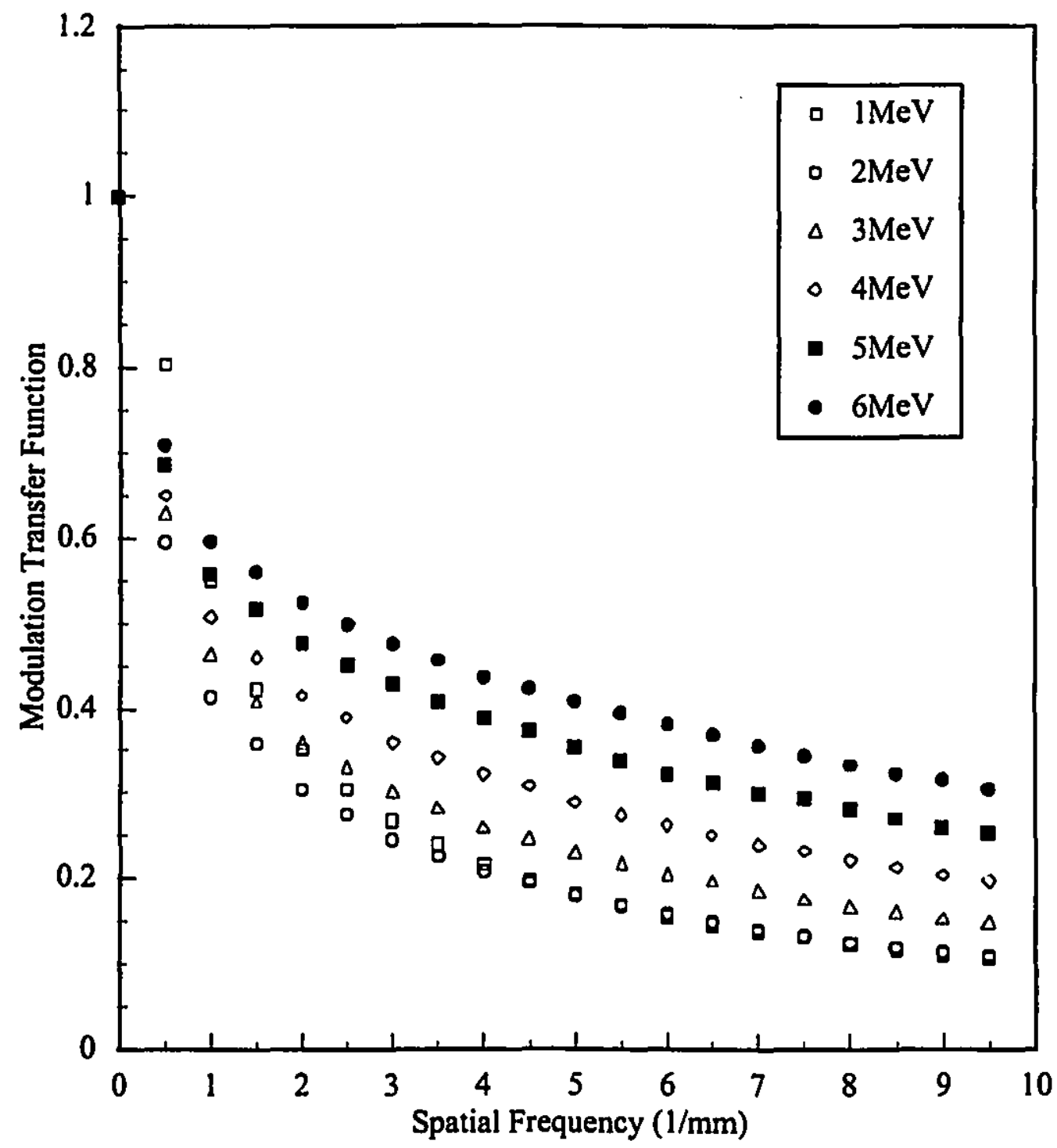

Figure 2.7. The modulation transfer functions of a $0.3 \mathrm{~mm}$ a-Se/1 mm Cu receptor at various photon energies. 


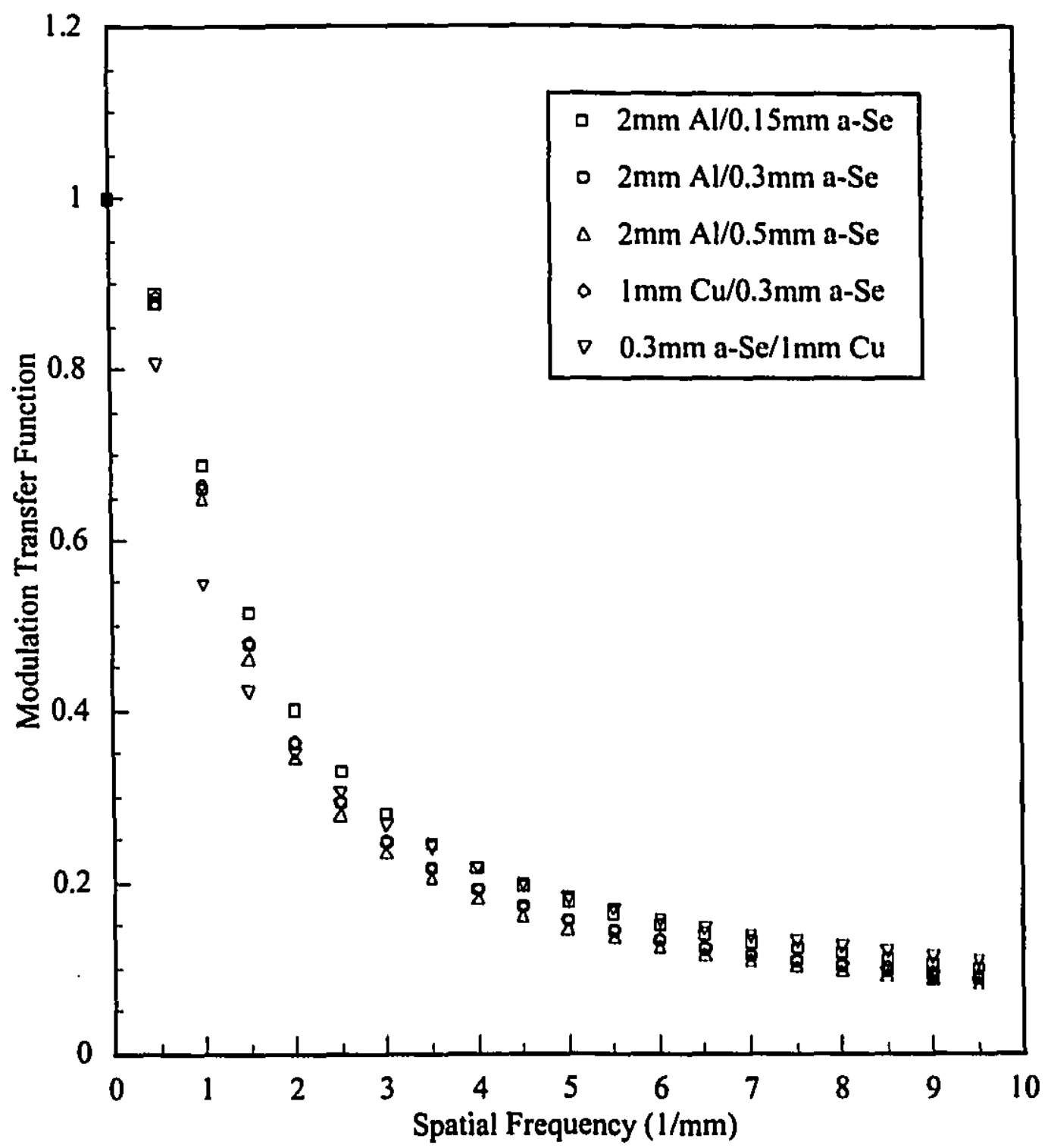

Figure 2.8. The modulation transfer functions of four receptor at incident photon energy of $1 \mathrm{MeV}$. 


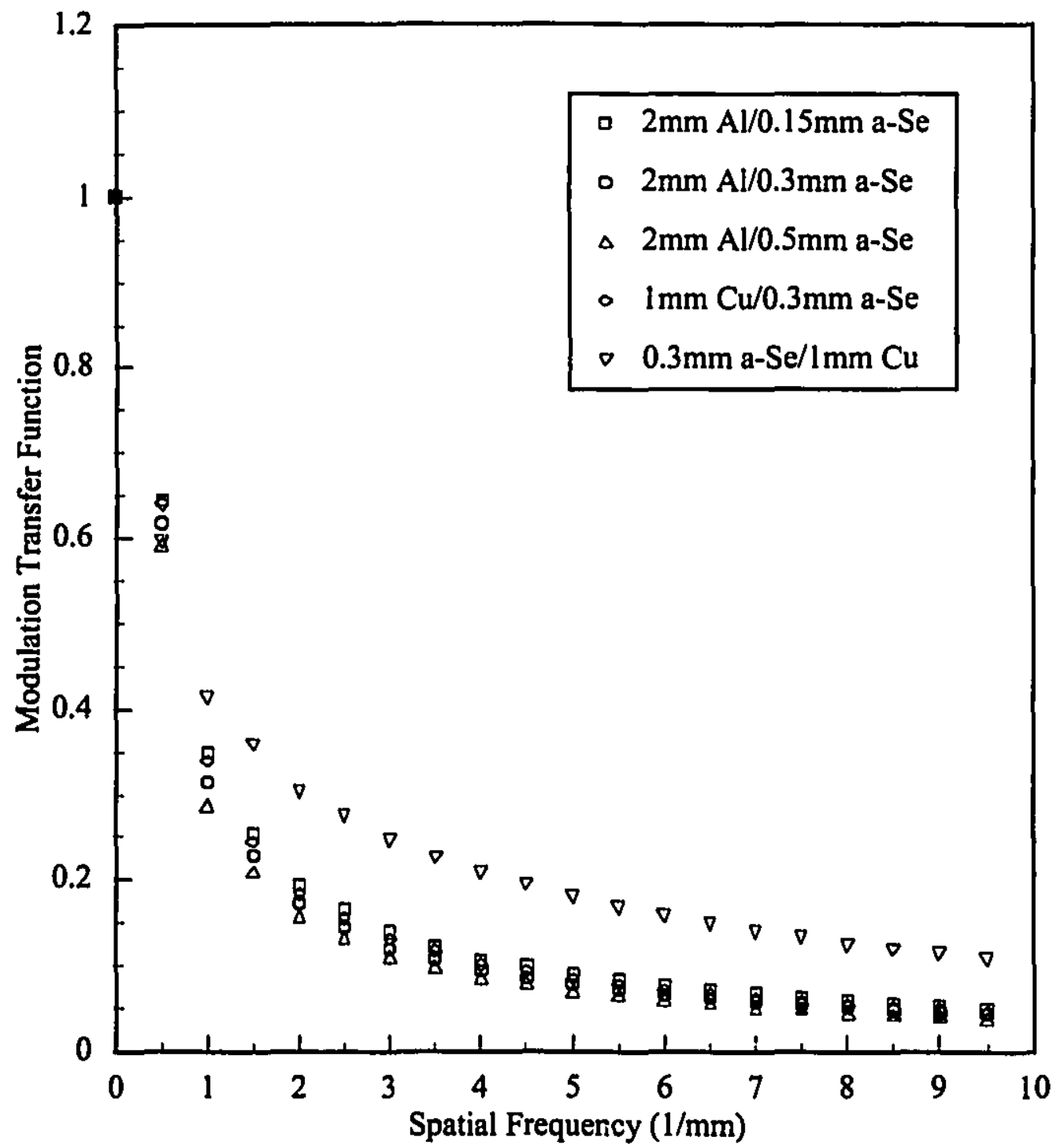

Figure 2.9. The modulation transfer functions of four receptor at incident photon energy of $2 \mathrm{MeV}$. 


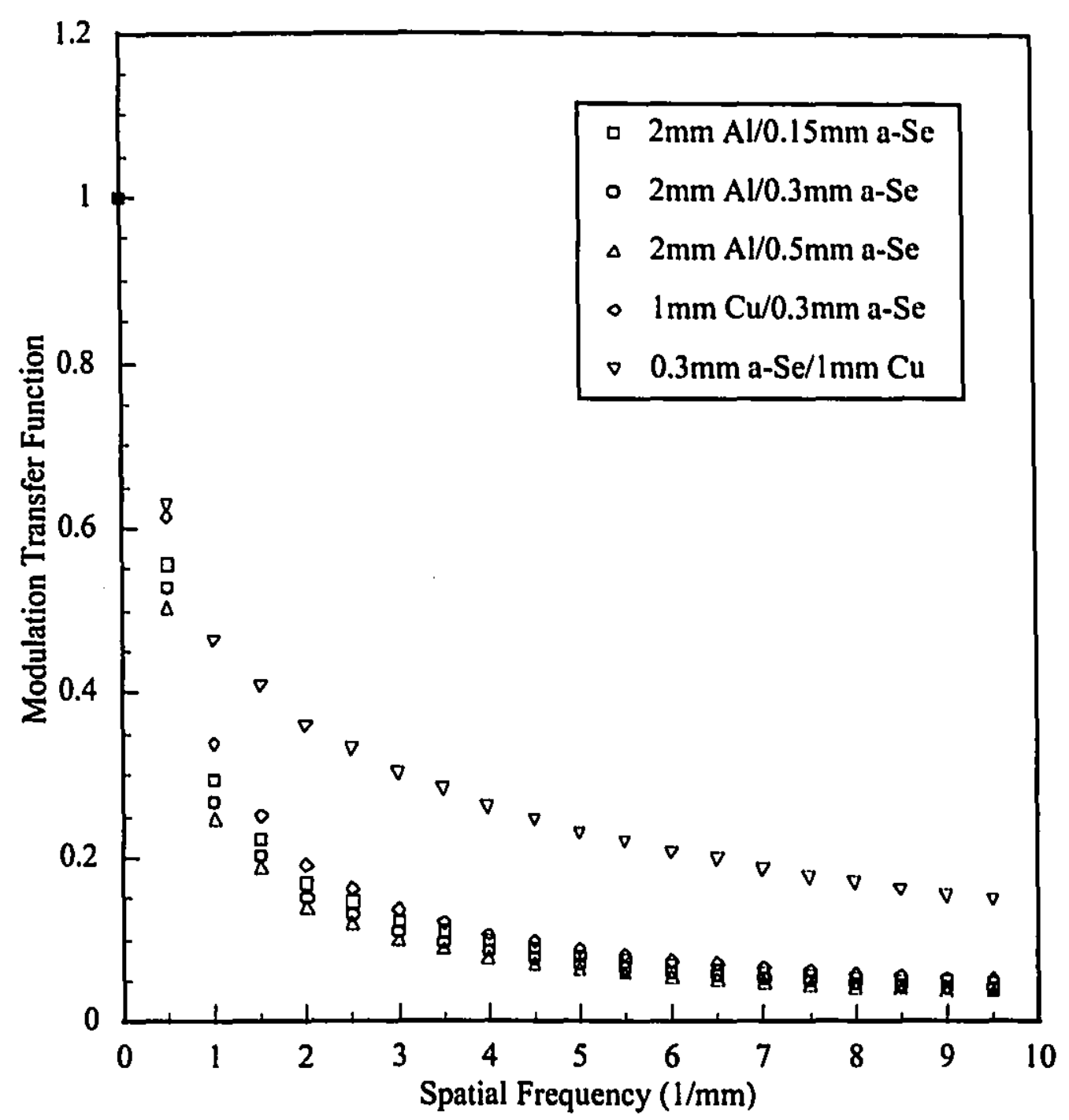

Figure 2.10. The modulation transfer functions of four receptor at incident photon energy of $3 \mathrm{MeV}$. 


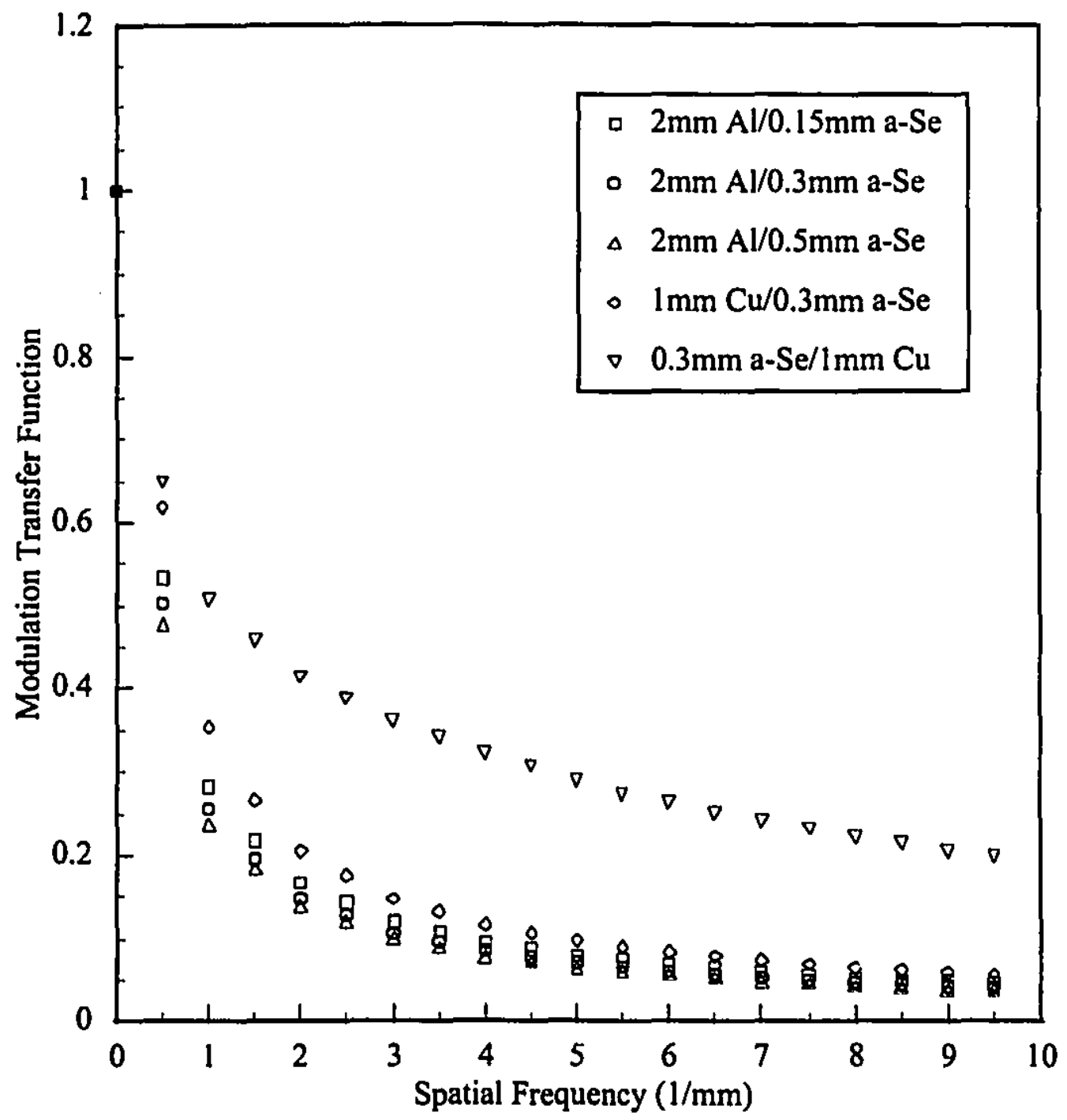

Figure 2.11. The modulation transfer functions of four receptor at incident photon energy of $4 \mathrm{MeV}$. 


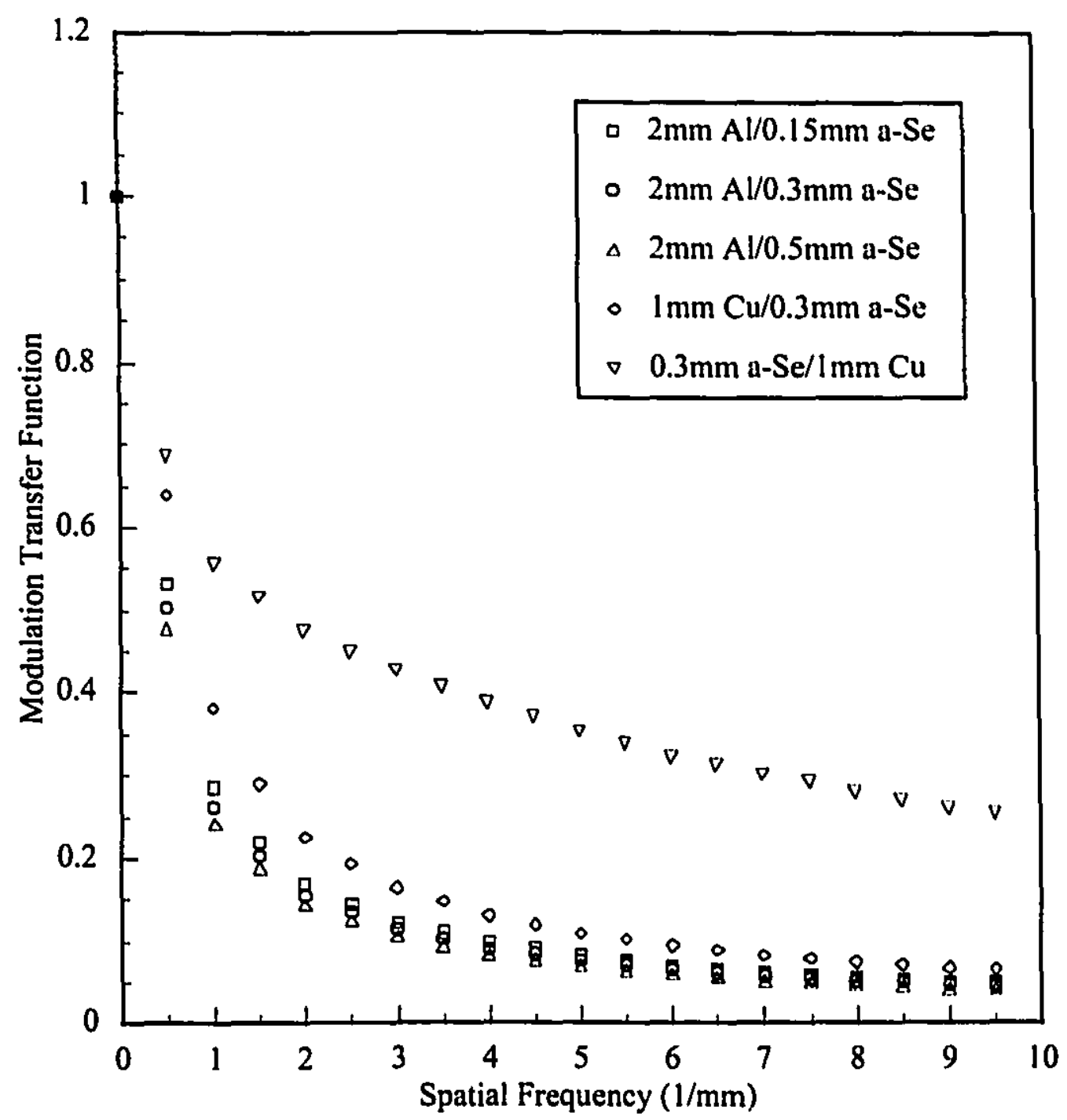

Figure 2.12. The modulation transfer functions of four receptor at incident photon energy of $5 \mathrm{MeV}$. 


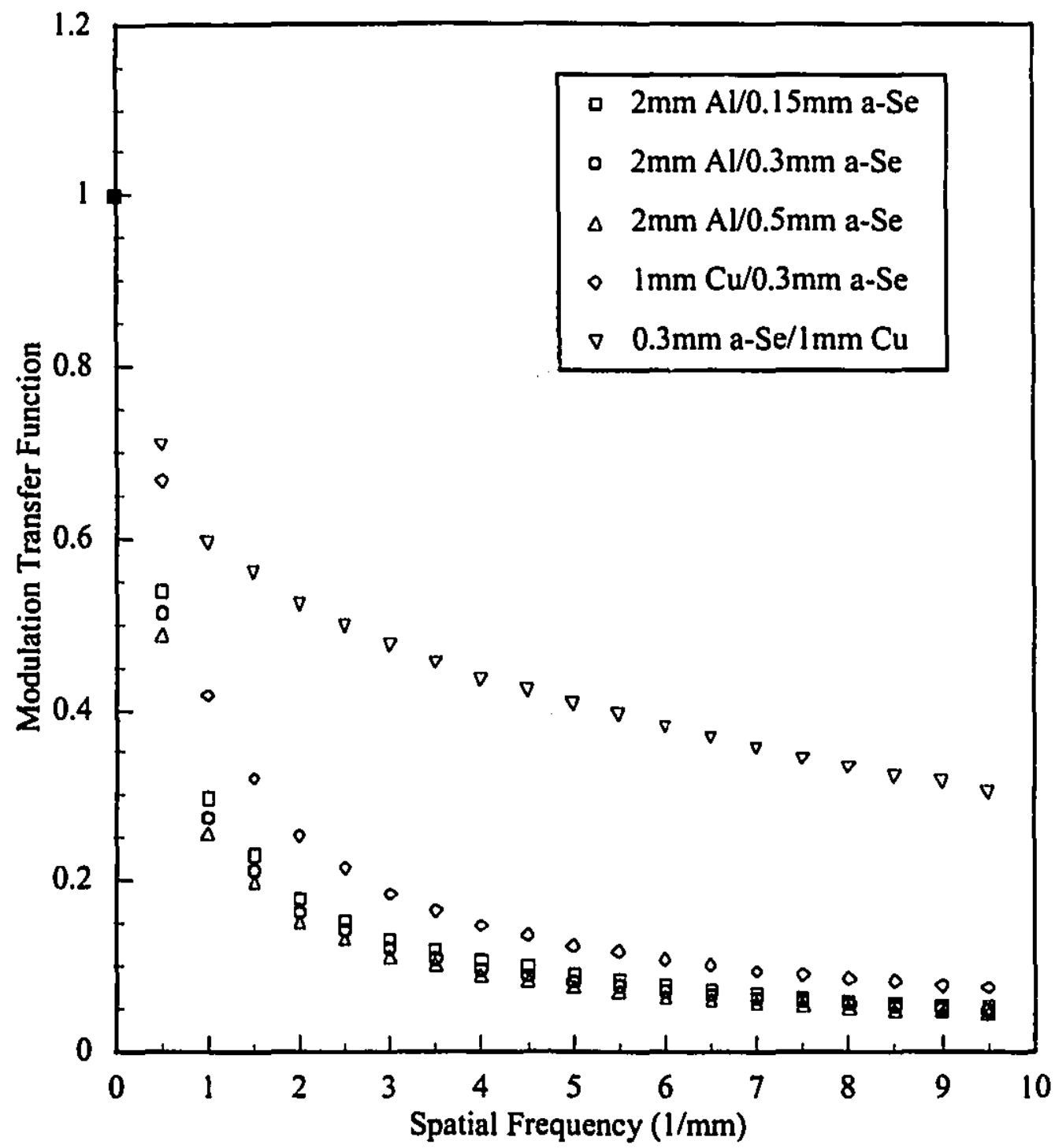

Figure 2.13. The modulation transfer functions of four receptor at incident photon energy of $6 \mathrm{MeV}$. 
a $1 \mathrm{~mm}$ thick $\mathrm{Cu}$ front plate and $300 \mu \mathrm{m}$ thick a-Se. The calculated MTFs are shown in Figures 2.3 to 2.6. Error bars are not plotted because they are smaller than the symbols.

For each plate, it appears that the MTF degrades as energy increases and becomes relatively constant from $2 \mathrm{MeV}$ up to $\sigma \mathrm{MeV}$. This appears to indicate that there is a transition of the dominant interaction from one type to another between 1 and $2 \mathrm{MeV}$. The MTFs were also calculated for the a-Se/Cu receptor when the $\mathrm{Cu}$ plate was used as back plate (Figure 2.7). Degradation was also observed as the photon energy was increased.

To examine the effects of the front metal plate and of the thickness of the a-Se, the data of all receptors at each individual energy were plotted in Figures 2.8 to 2.13 . It can be seen that for the Al plate receptors, the MTF decreases as the thickness of the a-Se increases at all energies $(1 \sim 6 \mathrm{MeV})$. For the $300 \mu \mathrm{m}$ thick a-Se layer, the $2 \mathrm{~mm}$ Al plate and the $1 \mathrm{~mm} \mathrm{Cu}$ plate lead to the same modulation transfer function at $1 \mathrm{MeV}$. As the photon energies increases, the $\mathrm{Cu}$ plate improves the MTF considerably. When a back $\mathrm{Cu}$ plate is used, the MTF is the lowest at $1 \mathrm{MeV}$ but the highest from $2 \mathrm{MeV}$ up.

\section{$\S \S$ 2.6.2 Quantum Absorption Efficiency}

The quantum absorption efficiency is defined as the ratio of the photons that have deposited energy in the sensitive volume of the detector to all the incident photons. It represents the probability for an incident photon to deposit energy in the a-Se layer. Figure 2.14 shows the calculated quantum absorption efficiencies of four receptors. The 
error bars are too small to be shown in the plots. As expected, the quantum absorption efficiency increases as the a-Se layer becomes thicker when the same front plate is used. At $1 \mathrm{MeV}$, a front metal plate reduces the probability of absorption due to the attenuation of the primary beam. For energies $\geq 2 \mathrm{MeV}$, the $1 \mathrm{~mm} \mathrm{Cu}$ front plate increases the absorption more than the $1 \mathrm{~mm} \mathrm{Cu}$ back plate. A $1 \mathrm{~mm} \mathrm{Cu}$ back plate is more effective in absorption than a $2 \mathrm{~mm} \mathrm{Al}$ front plate.

\section{\$§ 2.6.3 Detector Response}

The output signal of a receptor is determined by the average energy deposited by an incident photon. Figure 2.15 shows the responses of four receptors to monoenergetic photons at different energies. Error bars are not shown because they are too small. For the three Al plates, detector response increases with the thickness of a-Se. For the same thickness of the a-Se layer $(300 \mu \mathrm{m})$, a $1 \mathrm{~mm} \mathrm{Cu}$ front plate results in a much greater detector response than a $2 \mathrm{~mm} \mathrm{Al}$ front plate. The comparative detector response of the $\mathrm{Cu}$ with respect to .1 iiivreases at higher energies. From $2 \mathrm{MeV}$ and up, it becomes even greater than that of the $\mathrm{Al}$ receptor with a thicker a-Se layer $(500 \mu \mathrm{m})$. Among all the receptors, tli: one with a $1 \mathrm{~mm} \mathrm{Cu}$ back plate has the lowest detector response.

\section{$\$ \S$ 2.6.4 Statistical Factor}

The statistical factor describes the loss in DQE due to the incomplete absorption of an interacting photon. As shown in Figure 2.16, the statistical factor of a front metal plate 


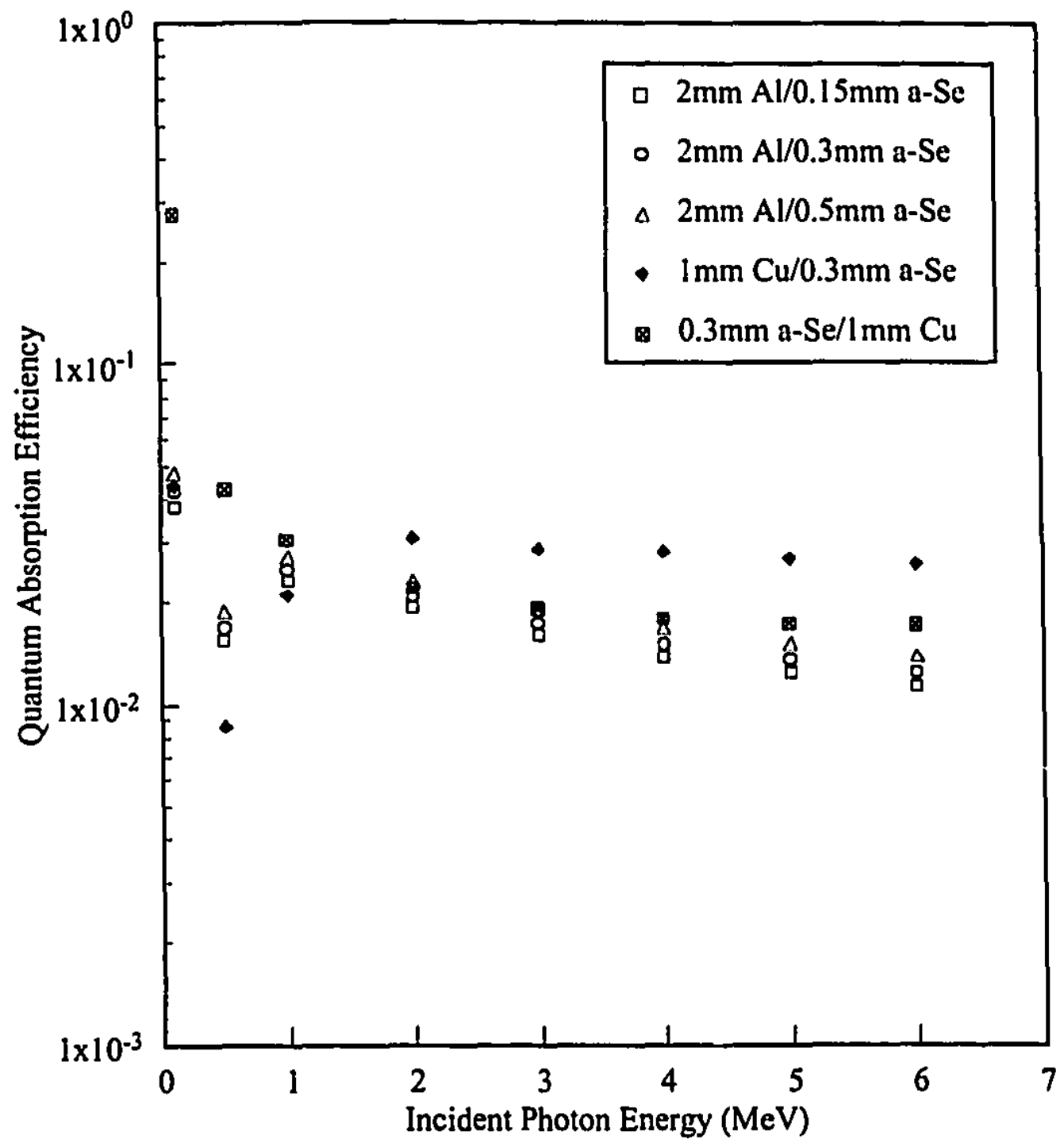

Figure 2.14. Quantum absorption efficiencies of four receptors at various incident photon energies. 


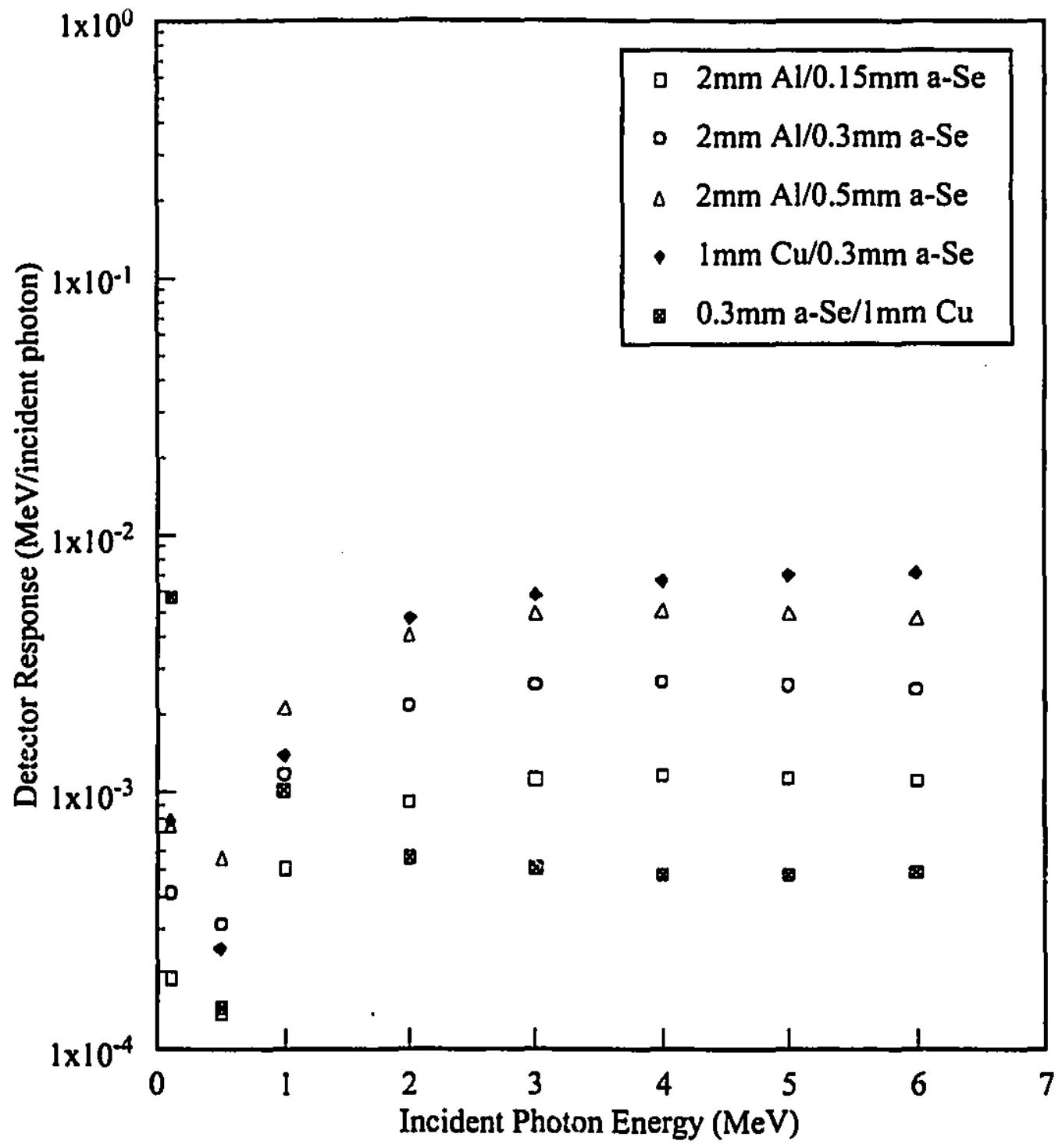

Figure 2.15. Detector response of four receptors at aifferent various incident photon energies. 


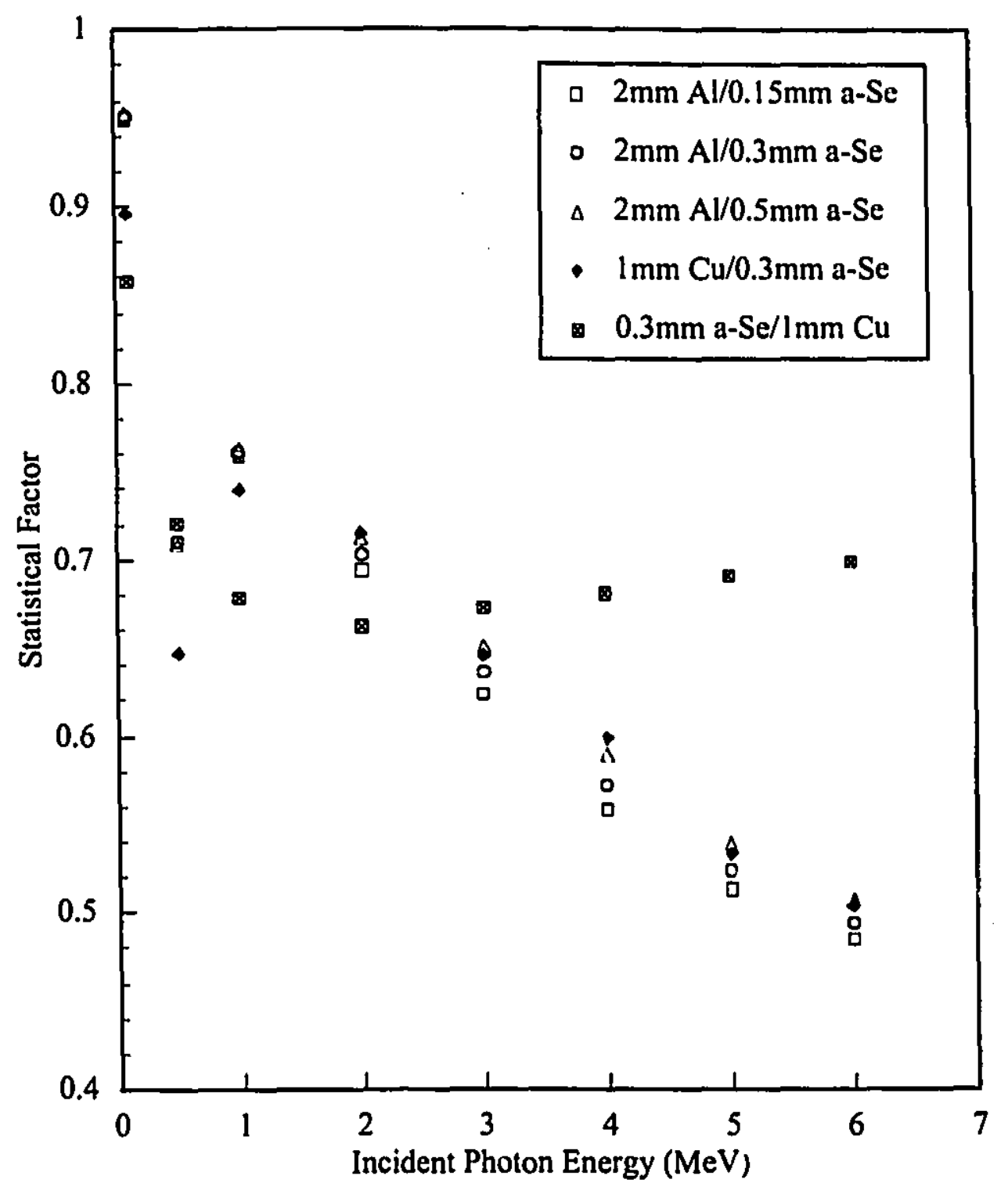

Figure 2.16. Statistical factors of four receptors at different incident photon energies. 
receptor decreases as the $\mathrm{x}$-ray energy increases. Error bars aie not plotted because they are too small. With the front metal plate as an electron convertor, the pulse height spectra of energy deposition in the a-Se layer have similar shapes at different energies. But the width increases with energy. The drop of the statistical factor is due to this widening. For a back plate receptor, however, the pulse height spectrum becomes narrower when x-ray energy increases. The smaller variation in the amount of the energy deposited per interaction photon is responsible for the slight increase of the statistical factor of the 1 $\mathrm{mm} \mathrm{Cu}$ back plate receptor.

\section{$\S \S$ 2.6.5 Detective Quantum Efficiency}

The zero spatial frequency DQEs for the four receptors are shown in Figure 2.17. Error bars are too small to be plotted. Except for the $1 \mathrm{~mm} \mathrm{Cu}$ receptor at $1 \mathrm{MeV}$, the DQEs of all four receptors decrease as incident photon energy increases. For the same front metal plate, a larger sensitive volume results in a higher $\mathrm{DQE}$ due a more complete absorption of the incident photon. For the same a-Se layer, a front metal plate decreases the DQE at $1 \mathrm{MeV}$ due to the attenuation of the primary photons (Figure 2.14). But from $2 \mathrm{MeV}$ up, the $1 \mathrm{~mm} \mathrm{Cu}$ front plate, and the $1 \mathrm{~mm} \mathrm{Cu}$ back plate, increase DQE more than the $2 \mathrm{~mm} \mathrm{Al}$ front plate. Figures $2.14,2.16$ and 2.17 indicate that $\mathrm{DQE}$ is dominated by the quantum absorption efficiency. The $D Q E$ as a function of spatial frequency can be calculated from Eq. (2.22). From $2 \mathrm{MeV}$ up, both the zero frequency DQE and the 


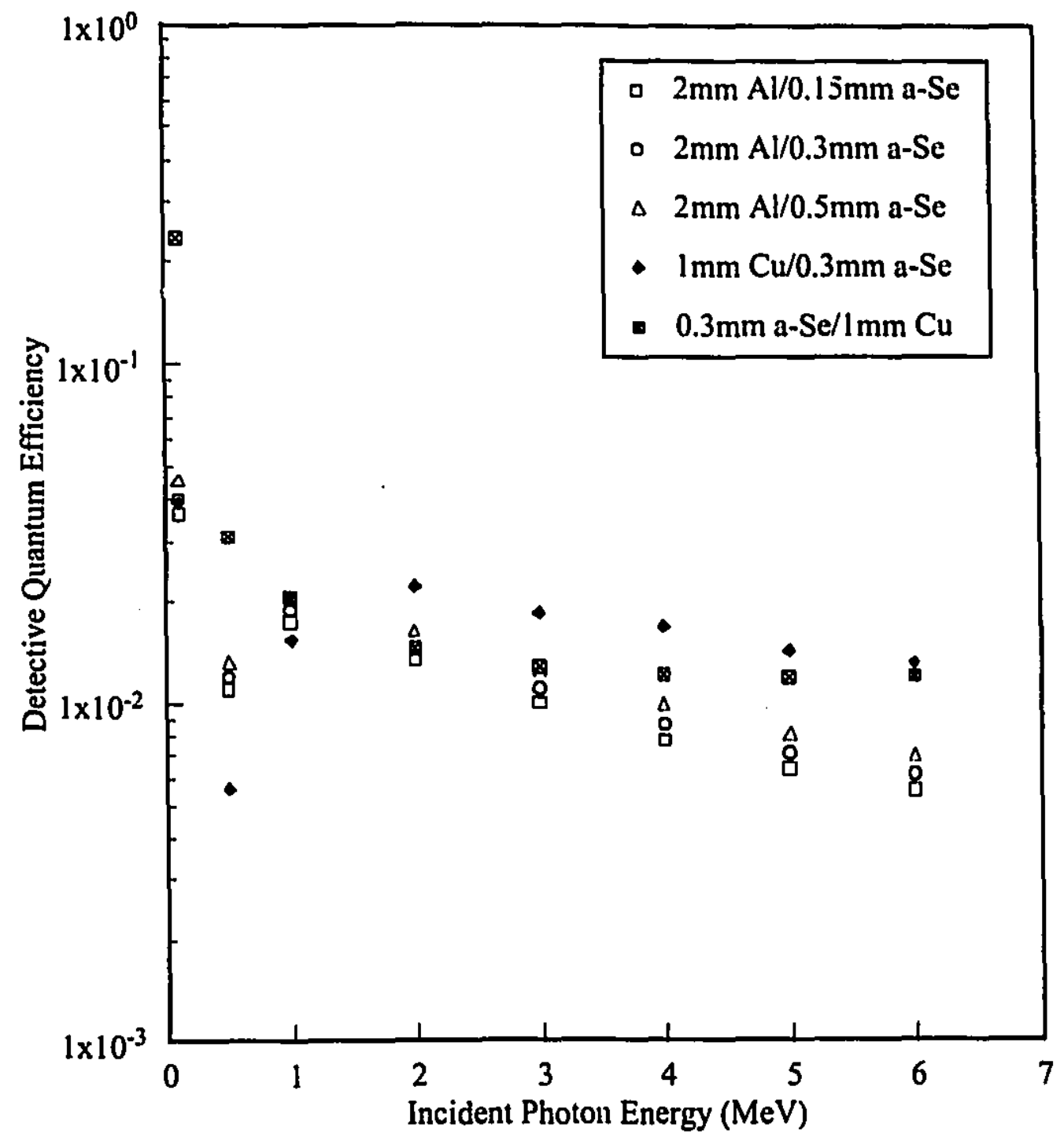

Figure 2.17. Zero spatial frequency detective quantum efficiency of four receptors at various incident photon energies. 
MTF of the $\mathrm{Cu}$ receptor are greater than those of the $\mathrm{Al}$ receptor. The $\mathrm{Cu}$ front plate, therefore, will lead to higher $\mathrm{DQE}$ at any detail level. At $1 \mathrm{MeV}$, the $\mathrm{DQE}$ of the $\mathrm{Cu}$ receptor at higher spatial frequencies will be compensated by its higher MTF.

\section{$\S 2.7$ Conclusions}

We have calculated the modulation transfer function, the detector response and the detective quantum efficiency of four metal/a-Se image receptors in megavoltage imaging. These imaging characteristics can reveal the effects of detector geometry and composition on the performance of a receptor in terms of contrast, spatial resolution and noise level.

Spatial resolution decreases drastically as energy increases from $1 \mathrm{MeV}$ to $2 \mathrm{MeV}$ and becomes relatively constant from $2 \mathrm{MeV}$ up. A thicker a-Se layer will also degrade spatial resolution. For the same metal, a front plate detector has better spatial resolution than a back plate one at $1 \mathrm{MeV}$. At higher energies, the opposite is true. This cannot be considered as an advantage of a back plate receptor because the sensitive volume will be exposed to scattered radiation. For receptors with the same a-Se layer, a $\mathrm{Cu}$ front plate can lead to better spatial resolution than an $\mathrm{Al}$ one for energies ranging from 1 $\mathrm{MeV}$ to $6 \mathrm{MeV}$.

The detective quantum efficiency decreases by approximately $30 \%$ from $1 \mathrm{MeV}$ to 6 MeV. It is tffected by the size of the sensitive volume of a detector and the metal build up layer. A front metal plate can increase the $D Q E, s c$ does a larger sensitive volume. 
The enhancement by the front metal plate is much more significant than that achieved by a thicker a-Se layer. Studies on the effects of the geometry and the composition of the detector show that the quantum detective efficiency appears to be dominated by the quantum absorption efficiency. A heavier metal is more effective in increasing the quantum absorption efficiency and consequently increasing the detective quantum efficiency.

Image formation in a xeroradiograhic system has three stages: $\mathrm{x}$-ray absorption, electron-hole pair production and charge collection. In our simulations, only the first stage is modeled. Another simplification is that only monoenergetic $\mathrm{x}$-ray beams were considered because the spectrum of beam transmitted through the patient is unknown.

Our calculations of zero frequency DQEs have followed the approach taken by Jaffray et al to calculate zero frequency DQEs of metal plate/phosphor screen combinations. Since the physical process modeled by the simulations is the same: energy absorption, the results could be compared. In fact, similar trends are observed in the way zero frequency $\mathrm{DQE}$ changes with respect to incident photon energy although the behavior of the DQE(0) of the metal plate/a-Se detector is not as simple. Since the Monte Carlo results are the higher limit, comparison of the overall performance of the two type of detection systems (metal/a-Se versus metal/phosphor) must include the later stages in the imaging chain. The metal/phosphor system requires an additional component that converts light into an 
electronic signal which may seriously affect the resultant DQE. This critical component is theoretically not required for metal/a-Se system, where the information is already stored in charge form. Intuitively, it should be simpler to read charge information directly in the metal/a-Se system than it would be with a metal/phosphor system. However, the optimum technique for reading a metal/a-Se has not been found yet.

Although the results are the higher limits of the detector considered, the objective of this study is to investigate the effect of the detector composition and geometry on the imaging characteristics. Our results seem to indicate that detector response and noise level are the two important factors in the design of an amorphous selcnium receptor for electrostatic portal imaging. Therefore the advantage in developing such a portal imaging device is to reduce noise level and imaging dose. 


\section{References}

I R. Swank, "Absorption and noise in X-ray phosphors," Journal of Applied Physics 44, 4199-4203 (1973).

$2 R$. Swank, "Measurement of absorption and noise in an x-ray image intensifier," Journal of Applied Physics 45, 3673-3678 (1974).

${ }^{3}$ C. E. Dick and J. W. Motz, "Image information transfer properties of x-ray image intensifiers," Medical Physics 10, 337-346 (1981).

4 R. T. Droege and B. Bjarngard, "Influence of metal screens on contrast in megavoltage x-ray imaging," Medical Physics 6, 515-518 (1979).

5 D. A. Jaffray, K. Chawla, C. Yu, and J. W. Wong, "Dual beam imaging for online verification of radiotherapy field placement," Int. J. Radiat. Oncol. Biol. Phys. 33, $1273-1280$ (1995)

6 B. G. Fallone, 'r. Faico, H. Wang, and N. Araj, "An electrostatic-based detector for portal imaging," in The 4th International Workshop on Electronic Portal Imaging: Portal Imaging in Theory and Practice (Amsterdam, Holland ,1996).

7 B. G. Fallone and T. Falco, "Megavoltage imaging method using a combination of a photoreceptor with a high energy photon converter and intensifier," International Patent File (1996). 
8 T. Villafana, "Modulation transfer function of a finite scanning microdensitometer slit," Medical Physics 2, 251-54 (1975).

${ }^{9}$ D. W. O. Rogers and A. F. Bielajew, "Monte Carlo techniques of electron and photon transport for radiation dosimetry," The Dosimetry of Ionizing Radiation III (editors: Kenneth R. Kase, Bengt E. Bjarngard, and Frank H. Attix, Academic Press, San Diego, California 1990).

10 A. F. Bielajew and. D. W. O. Rogers, "PRESTA-the parameter reduced electronstep transport algorithm for electron monte carlo transport," Nuclear Instruments and Method B18, 535-548 (1984). 


\section{Chapter 3 Sensitivity and Contrast-Detail Studies}

$\$ 3.1 \quad$ Introduction $\ldots \ldots \ldots \ldots \ldots \ldots \ldots \ldots \ldots \ldots \ldots$

$\$ 3.2$ System Overview $\ldots \ldots \ldots \ldots \ldots \ldots \ldots \ldots \ldots \ldots$

$\S \S 3.2 .1$ The Detector Unit . . . . . . . . . . . . . 61

$\S \S 3.2 .2$ The Electronics System $\ldots \ldots \ldots \ldots \ldots \ldots$

$\S \S 3.2 .3$ The Host Computer . . . . . . . . . . . . . 63

$\S 3.3$ Electrostatic Image Formation $\ldots \ldots \ldots \ldots \ldots \ldots$

$\S \S 3.3 .1 \quad$ Charging $\ldots \ldots \ldots \ldots \ldots \ldots \ldots \ldots \ldots \ldots \ldots$

$\S \S 3.3 .2$ Receptor Orientation $\ldots \ldots \ldots \ldots \ldots \ldots \ldots$

$\S 3.4$ Image Acquisition $\ldots \ldots \ldots \ldots \ldots \ldots \ldots \ldots \ldots$

$\S 3.5$ Sensitivity Curve Measurement and Modelling $\ldots \ldots \ldots \ldots \ldots$

$\S \S 3.5 .1 \quad$ Radiation Discharging $\ldots \ldots \ldots \ldots \ldots \ldots$

$\S \S 3.5 .2$ Monoenergetic Photons . . . . . . . . . . . 67

$\S \S$ 3.5.3 Polyenergetic Photons $\ldots \ldots \ldots \ldots \ldots \ldots$

$\S 3.6$ Phantom Tests $\ldots \ldots \ldots \ldots \ldots \ldots \ldots \ldots \ldots$

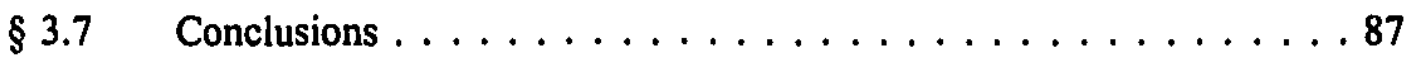




\section{§ 3.1 Introduction}

Electrostatic imaging is a process in which the intensity pattern of a photon beam is transformed to a charge distribution on the surface of a photoconductor.' This imaging modality referred to as xeroradiography was introduced into radiography in the early 70s for acquiring mammographic images. Xeroradiography has also been employed by Wolfe ${ }^{2}$ to acquired portal images in radiotherapy. Despite the high quality inherent in the latent charge image on the photoconductor, usually amorphous selenium (a-Se), the performance of xeroradiographic imagers was limited by the powder cloud development method. ${ }^{3}$ With the development of novel methods for extracting the latent image, such as photoinduced discharge with laser ${ }^{4-6}$ and electrostatic coupling, xeroradiography is regaining its vitality. Recent studies have shown that electrostatic imaging by using a-Se and digital readout has various advantages over screen-film systems in mammography: higher contrast, wider dynamic range and improved quantum detective efficiency. 7,8 One would naturally consider the introduction of a-Se into portal imaging where beam energy is much higher. In this chapter, we will present a prototype portal imager vased on metal/a-Se and evaluate its performance in terms of sensitivity, noise level and spatial resolution.

\section{§ 3.2 System Overview}

As shown in Figure 3.1, the prototype portal imager consists of three major 

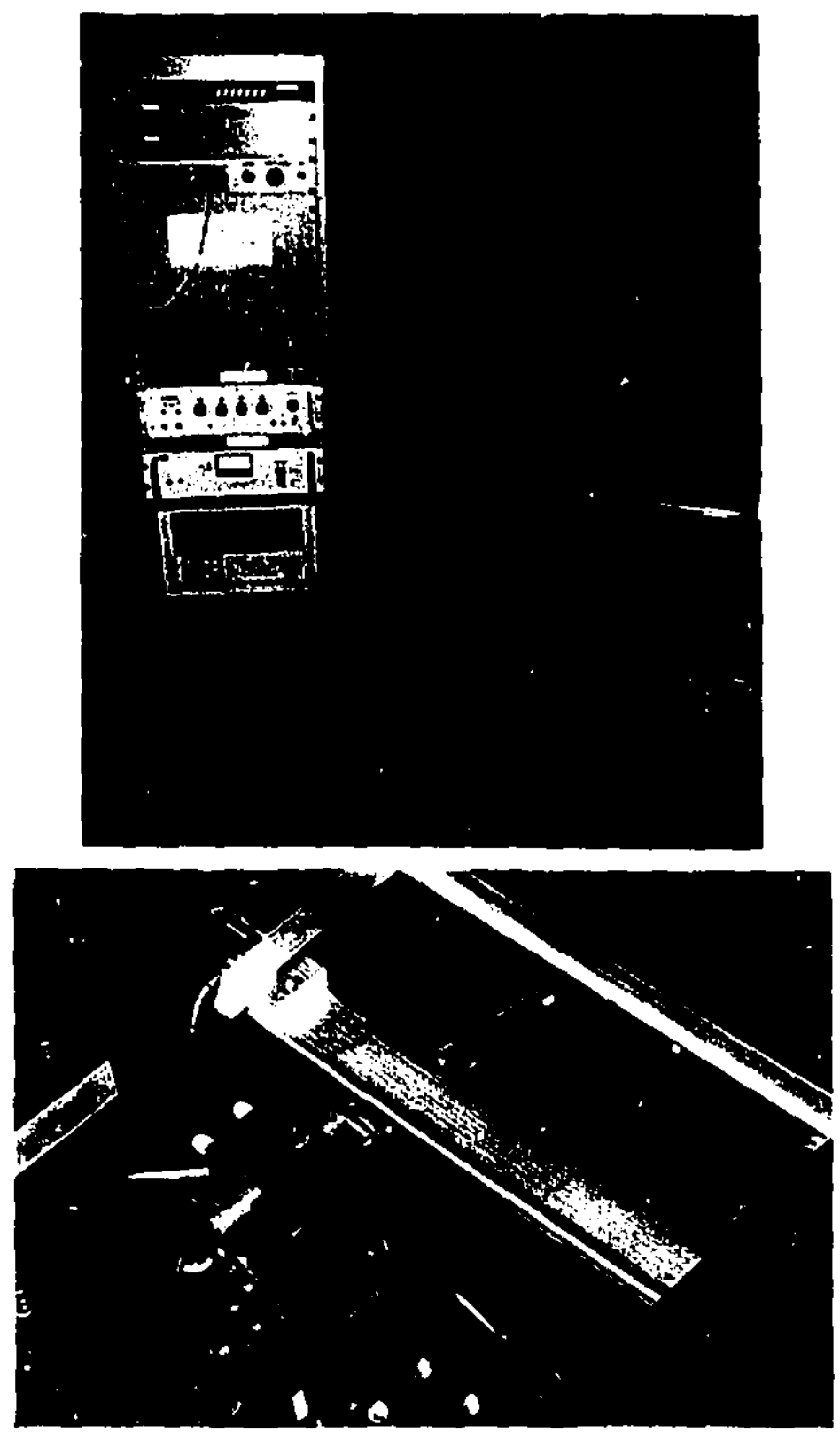

Figure 3.1. Major components of the prototype portal imager. 
components: a detector unit, an electronics system for the extraction of the latent image, and a host computer controlling the image acquisition. The detector unit and the electronics system are located in the radiation treatment suite while the host computer is located in the monitoring area.

\section{§§ 3.2.1 The Detector Unit}

The schematics of the detector unit is shown in Figure 3.2. Built within a lighttight box, a two level rack secured onto the bottom of the box serves as the base of the detector unit. The rack has two aluminum slabs forming the bottom and the top which are connected at the corners by four supporting columns made of steel. At the center of the top slab, there is a $8 \times 8 \mathrm{in}^{2}$ window with an insulating frame holding the image receptor: a metal plate coated with a thin layer of amorphous selenium. A two dimensional motion stage (Aerotech, Inc., Pittsburgh, PA) is immobilized on the bottom slab of the rack to provide the motion of scanning. The motion stage can accelerate to a constant velocity $(0 \sim 101.6 \mathrm{crn} / \mathrm{s}$ in $10 \mathrm{~ms})$, with an accuracy of $2 \mu \mathrm{m}$. A scorotron and an electrometer probe (Trek, Inc., Medina, NY) are installed on the motion stage. The probe has an aperture of $200 \mu \mathrm{m}$, rests at a distance of about $200 \mu \mathrm{m}$ from the a-Se surface and can measure potentials up to $3000 \mathrm{~V}$.

\section{§ 3.2.2 The Electronics System}

Hosted in a mobile cabinet, the electronics system includes two high veltage 


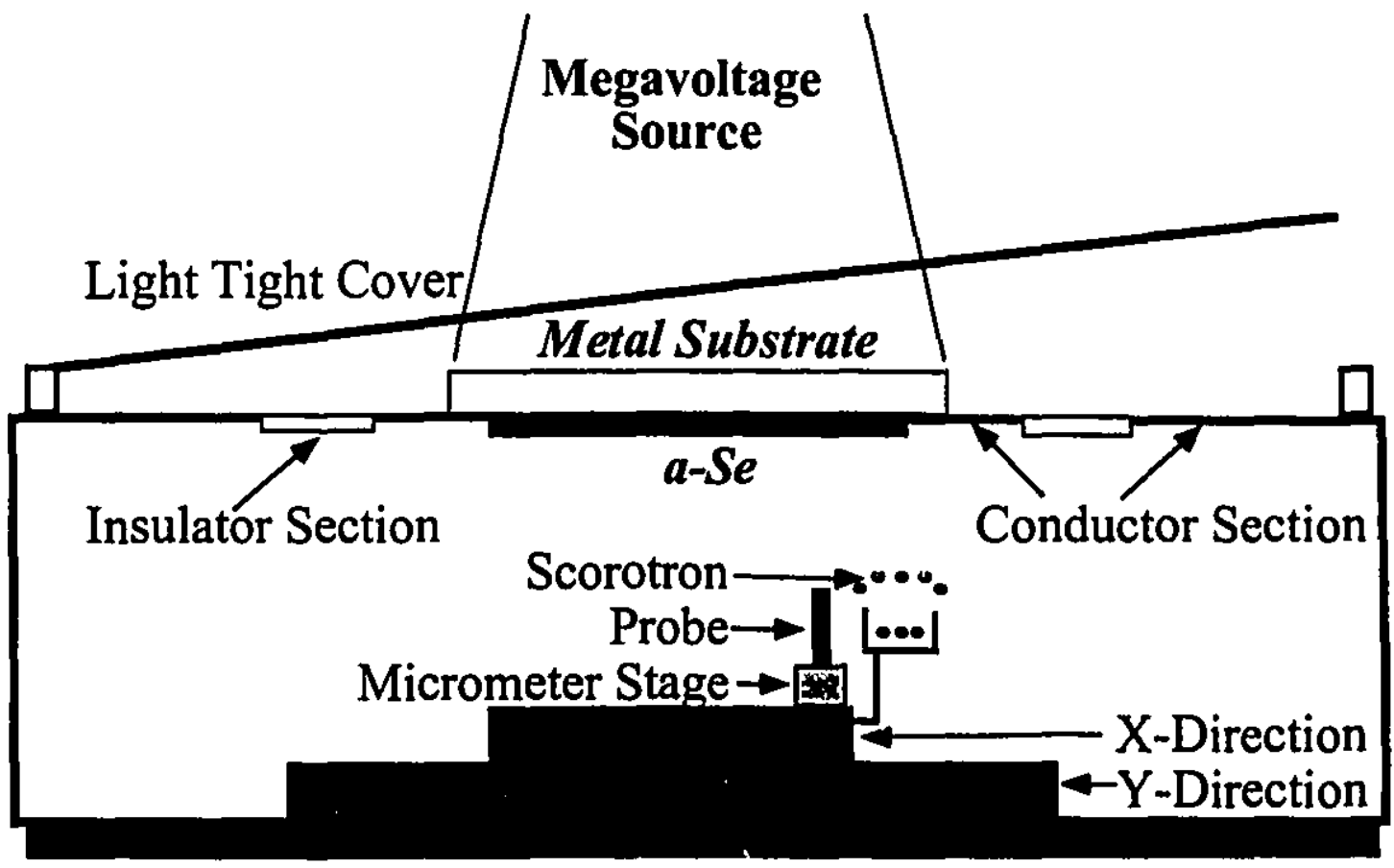

Light Tight Box

Figure 3.2. Schematics of the detector unit (Courtesy of Tony Falco). 
power supplies, an analog-to-digital converter (ADC488/16A) and a motion controller (Unidex 11, U11s). The ADC can scan and sample analog signals at rates up to $100 \mathrm{kHz}$ with 16 bits resolution. The high voltage power source is connected to the scorotron in the detector unit to provide corona.

\section{$\S \S$ 3.2.3 The Host Computer}

An IBM compatible personal computer with an 80386 CPU (Compaq Prolinea 4/100) running Microsoft Windows is employed to control the motion stage through the motion controller and to acquire data from the ADC. The motion controller and the ADC are interfaced with the host computer by a software package: LABVIEW (National Instruments, Inc, Austin, TX).

\section{§ 3.3 Electrostatic Image Formation}

The formation process of an electrostatic images has two steps: (a) the a-Se is charged in the dark to achieve a uniform charge distribution on its surface; (b) the image receptor is irradiated with a beam transmitted through an object. The varying intensity across the beam due to differential attenuation in the object causes spatially variant local discharging on the a-Se rurface resulting in a latent electrostatic image.

\section{§§ 3.3.1 Charging}

The most common charging method, coronal charging, is used in the prototype imager 
to achieve a uniform charge distribution on the a-Se surface. A sharp conductor on the scorotron is at a high electric potential (thousands of volts) to serve as a charge source which points to the a-Se on a grounded metal plate. The extremely strong electric field around the tip of the sharp conductor causes dielectric breakdown of air to ireate charge carriers. A wire mesh at a bias potential is placed between the point charge source and the a-Se to homogenize the electric field immediately above the a-Se. The charge carriers, rrimarily $\mathrm{CO}_{3}{ }^{-}$, drift in the electric field to the a-Se surface and become trapped. After repetitive "sweep and spray" by the scorotron, the a-Se accumulates charge and builds up a uniform potential on its surface to equal the charge on the sharp cenductor. We charged the plate to $2100 \mathrm{~V}$.

\section{$\S \S$ 3.3.2 Receptor Orientation}

In conventional electrostatic imaging, the photoconductor is directly exposed to radiation. This receptor orientation will not provide images of high quality due to the way photons interact with matter at therapy energy. As described in Chapter 2, the higher penetrating ability of megavoltage photons makes the absorption at the surface very low which consequently reduces contrast. Moreover, scatter from the object will increase noise level and blur. For these two reasons, our receptor is placed with the metal plate facing the radiation beam. 


\section{§ 3.4 Image Acquisition}

After irradiation, the a-Se surface is scanned with the electrometer probe in a raster fashion. The motion is generated by the motion stage driven by step motors. A $5 \times 5 \mathrm{in}^{2}$ area is scanned to obtain $200 \times 200$ sample points resulting in $\sim 600 \mu \mathrm{m}$ intervals. There is some information loss, but an increase in the number of sarapled points would seriously increase the acquisition time. The scan is set at a moderate speed to ensure a sufficient accuracy of the motion. As a result, about 7 minutes are required to extract one image.

\section{§ 3.5 Sensitivity Curve Measurement and Modelling}

\section{$\S \S$ 3.5.1 Radiation Discharging}

A latent image on an amorphous selenium surface is formed via local neutralization of a uniform charge distribution achieved through the charging procedure (e.g., corona charging) before irradiation. The extent of this local neutralization is proportional to the number of electron-hole pairs generated by the radiation in a small volume. Therefore the varying intensity across the radiation beam exiting the patient will result in a charge distribution pattern on the amorphous selenium surface.

As a photoconductor, an amorphous selenium plate is a capacitor in the dark. A uniform charge distribution $\sigma$ on the plate will result in a voltage difference between 
the two surfaces:

$$
V^{\prime}=\frac{\sigma d}{\epsilon}
$$

where $d$ is the thickness of the plate and $c$ is the dielectric constant of amorphous selenium. X-ray irradiation on the plate will create electron-hole pairs inside the amorphous selenium and these charges will drift in the electric field towards opposite surfaces to neutralize the initial charge on the surfaces. The decrease of surface charge $\Delta \sigma$ is proportional to radiation dose $\Delta D$ and inversely proportional to the average energy required to generate and collect one electron-hole $r$ air in selenium $W_{ \pm}$:

$$
\Delta \sigma \propto-\frac{\Delta D}{W_{ \pm}}
$$

Considering that $W_{ \pm}$is dependent on the electric fieid in the selenium, ${ }^{9}$

$$
W_{ \pm} \propto\left(\frac{V}{d}\right)^{-\frac{2}{3}}
$$

the slope of discharging due to irradiation can be expressed as:

$$
\frac{\Delta V}{\Delta D} \propto-\frac{d^{\frac{1}{3}} V^{\frac{2}{3}}}{\epsilon}
$$

which will lead to the voltage difference between the two surfaces as a function of radiation dose:

$$
V(D)=V_{0}\left[1-\frac{\alpha d^{\frac{1}{3}}}{\epsilon V_{0}^{\frac{1}{3}}} \cdot D\right]^{3}
$$


where $\alpha$ is a proportional constant characterizing the photoconductor's sensitivity to $\mathrm{x}$ rays. The voltage drops to zero at

$$
D_{m}=\frac{\epsilon}{a} \cdot\left(\frac{V}{d}\right)^{\frac{1}{3}}
$$

which characterizes the dynamic range. The image contrast is determined by the discharging slope:

$$
\frac{d V}{d D}=-\frac{3 \alpha d^{\frac{1}{3}} V_{0}^{\frac{2}{3}}}{\epsilon} \cdot\left(1-\frac{D}{D_{m}}\right)^{2}
$$

which becomes less steep as the radiation dose increases. It is obvious that there is a trade-off between dynamic range and image contrast, and that these parameters depend on the thickness of the a-Se layer $d$, the $\mathrm{x}$-raj' energy absorption efficiency $\alpha$ and the initial voltage across the a-Se layer $V_{0}$. While the thickness of the a-Se layer $d$ and the initial voltage $V_{0}$ are original parameters, $\mathrm{x}$-ray energy absorption efficiency $\alpha$ is dependent on the thickness of the a-Se layer $d$ and the $\mathrm{x}$-ray energy. The dependence of the dynamic range and image contrast on $x$-ray absorption and the dependence of the absorption efficiency on the image receptor geometry can be investigated by measuring the discharging curve of a-Se by x-ray irradiation.

\section{$\S \S$ 3.5.2 Monoenergetic Photons}

There are usually two types of photon beams used in teletherapy: $\gamma$-rays from radioactive isotopes or $\mathrm{x}$ rays generated by medical linear accelerators. The same beams 
are also used for acquiring 1 ortal images. In order to isolate different factors involved in the experiment, we first measured receptor sensitivity to monoenergetic photons from $\mathrm{Co}^{60}$

Four receptors with different metal plates and different thicknesses of the a-Se layer were radiated on a T780 Cobalt Unit (Theratronics, Kanata, Ontario) at a source-toreceptor distance of $120 \mathrm{~cm}$. The radiation beam was coilimated such that the cross section of the beam on the receptor surface is a square. To examine the function of the metal plate as buildup layer, the receptors were radiated with the front plate orientation (the metal plate facing the beam) and the back plate orientation (the a-Se facing the radiation).

The measurement with the front plate orientation starts with coronal charging the receptor with the scorotron. Then the receptor is radiated with a preset radiation time. Scanring the receptor with the electrostatic probe after irradiation generates a map of potential of the a-Se surface. This procedure is repeated to obtain a series of open field images. The average pixel value with a small region of interest located at the center of each image is calculated and then converted to potential on the a-Se surface. This ensures that the measured potential will not be affected by dark decay.

The back plate orientation causes inconvenience in the measurement since the privtotype imager is designed for a front plate receptor. After charging, the receptor has 
to be iriverted manually before irradiation and then be inverted back for scanning. The inverting operations are performed in the dark with great care.

The measured radiation discharging curves of three aluminum plates and copper plate are shown individually in Figures 3.3 to3.6, and combined in Figure 3.7. Error bars are not plotted because they are too small. For all four plates the discharging is faster when the metal plate is used as a build-up layer. There is no significant difference between the copper plate and the aluminum plate with the same a-Se thickness although the discharging curve of the copper plate is slightly steeper. For the three aluminum plates, the discharging rate increases with the a-Se thickness. It appears that the thickness of the a-Se is a more sensitive factor than the thickness of the metal plate.

To quantify the receptor sensitivity in an absolute manner, the discharging process nee? to be characterized in terms of radiation dose to the receptor. The determination of this dose requires the achievement of electronic equilibrium in the a-Se. Because there is no justification of the existence of this equilibrium, we can only provide a relative dose to a small mass of tissue required to achieve the electronic equilibrium had it been radiated at the position of the image receptor. At the time of the measurements, this dose rate at the position of the receptor ( $120 \mathrm{~cm}$ from the source) was $37 \mathrm{cGy} / \mathrm{min}$.

The data are fitted to the following function

$$
V(D)=V_{0}\left[1-\frac{T}{T_{m}}\right]^{3}
$$




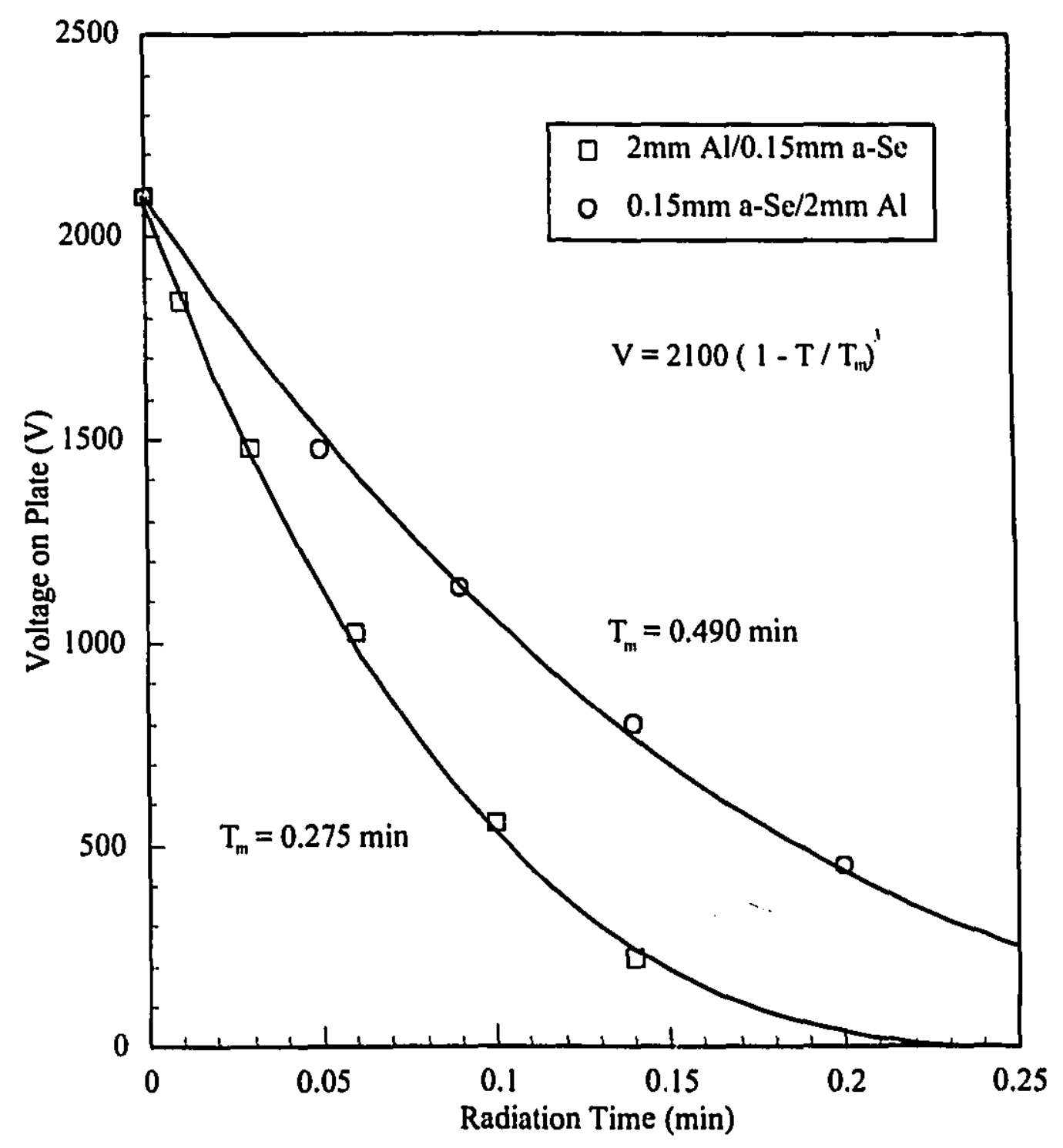

Figure 3.3. Sensitivity curves of a $2 \mathrm{~mm} \mathrm{Al} / 0.15 \mathrm{~mm}$ a-Se receptor measured on a T780 Cobalt Unit with the front plate and back plate configurations. The dose rate at the receptor is $37 \mathrm{cGy} / \mathrm{min}$. 


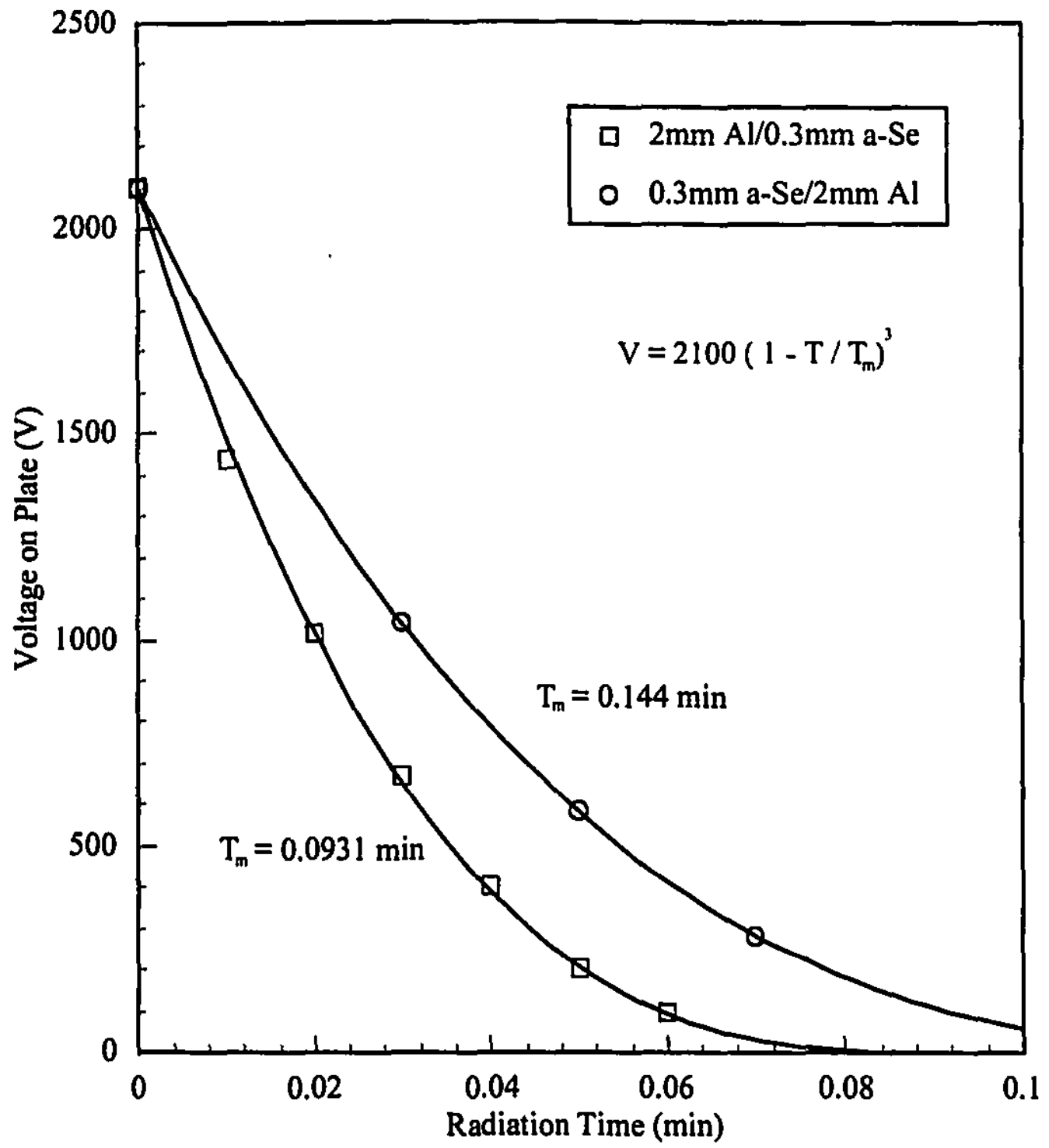

Figure 3.4. Sensitivity curves of a $2 \mathrm{~mm} \mathrm{Al} / 0.3 \mathrm{~mm}$ a-Se receptor measured on a T780 Cobalt Unit with the front plate and back plate configurations. The dose rate at the receptor is $37 \mathrm{cGy} / \mathrm{min}$. 


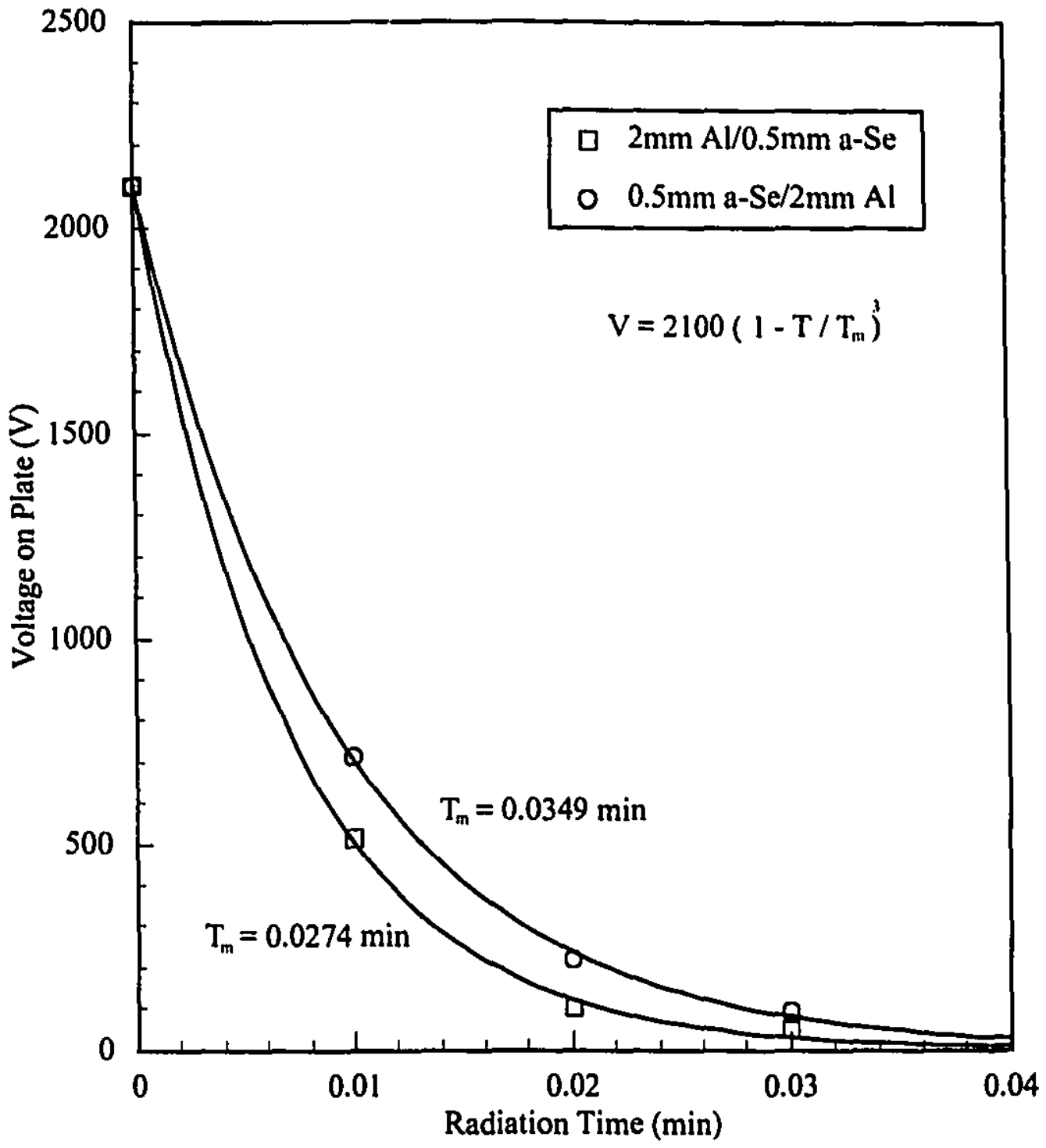

Figure 3.5. Sensitivity curves of a $2 \mathrm{~mm} \mathrm{Al} / 0.5 \mathrm{~mm}$ a-Se receptor measured on a T780 Cobalt Unit with the front plate and back plate configurations. The dose rate at the receptor is $37 \mathrm{cGy} / \mathrm{min}$. 


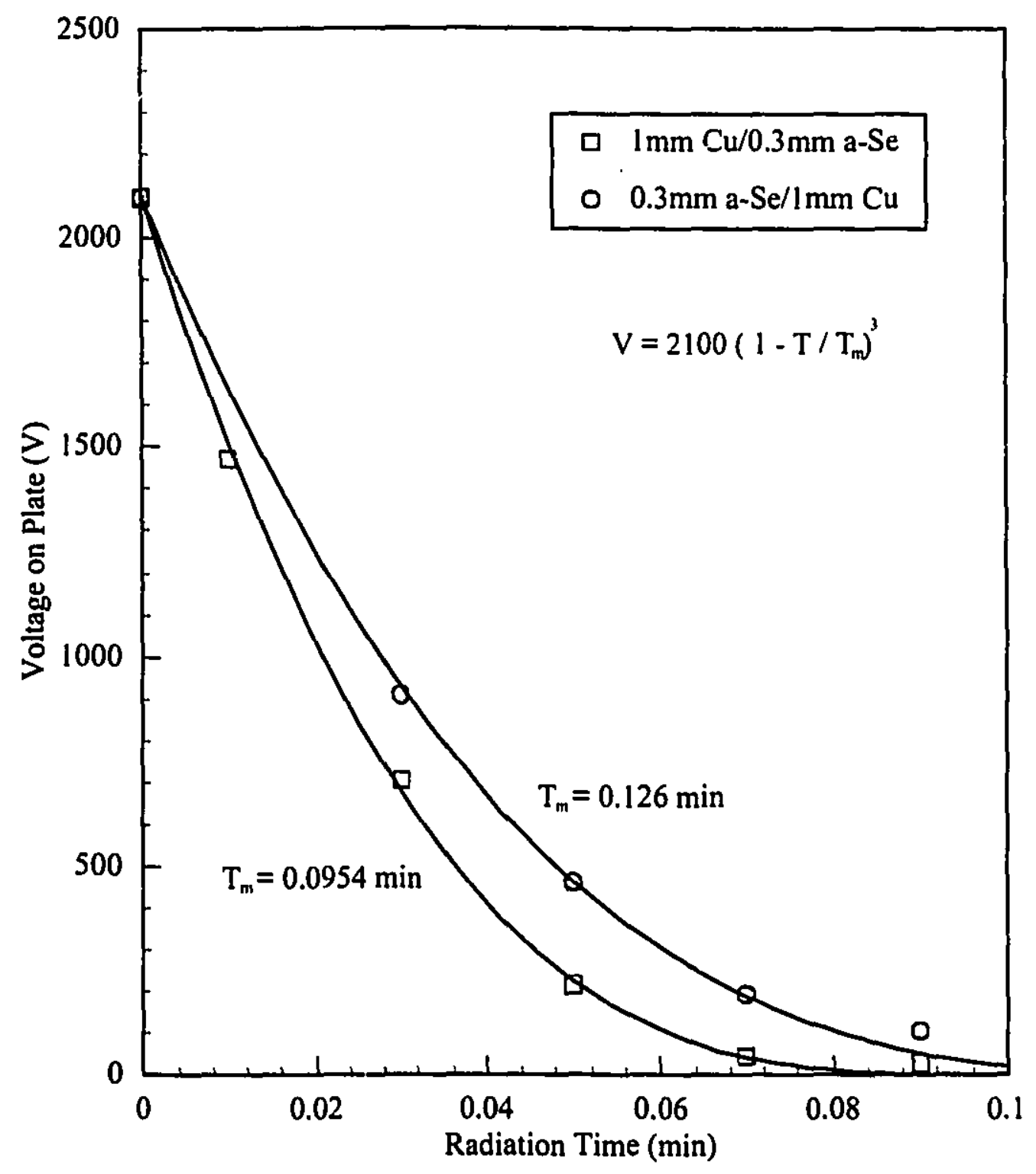

Figure 3.6. Sensitivity curves of a $1 \mathrm{~mm} \mathrm{Cu} / 0.3 \mathrm{~mm}$ a-Se receptor measured on a T780 Cobalt Unit with the front plate and back plate configurations. The dose rate at the receptor is $37 \mathrm{cGy} / \mathrm{min}$. 


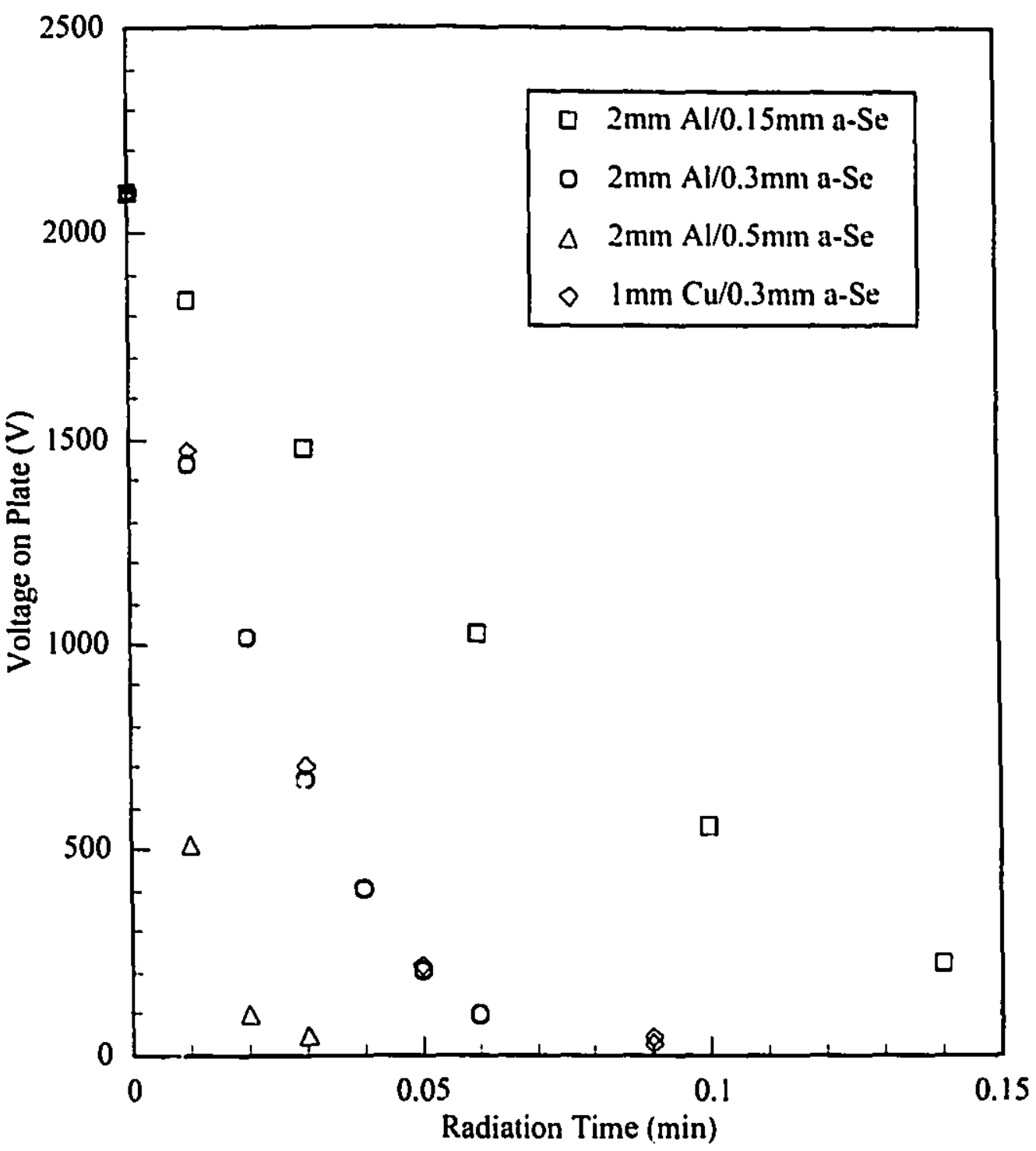

Figure 3.7. The sensitivity curves of four different a-Se receptors measured on a T780 Cobalt Unit. Dose rate at the receptor is $37 \mathrm{cGy} / \mathrm{min}$. 
Table 3.1 Radiation times required for compleie discharge of different receptors on T780 Cobalt Unit and correlation coefficients obtained from the modelling.

\begin{tabular}{|c|c|c|}
\hline Receptor & $T_{m}(\mathrm{~min})$ & $R$ \\
\hline $2 \mathrm{~min} \mathrm{Al} / 0.15 \mathrm{~mm} \mathrm{a-Se}$ & 0.275 & 0.999514 \\
\hline $0.15 \mathrm{~mm} \mathrm{a}-\mathrm{Se} / 2 \mathrm{~mm} \mathrm{Al}$ & 0.490 & 0.996700 \\
\hline $2 \mathrm{~mm} \mathrm{Al} / 0.3 \mathrm{~mm} \mathrm{a}-\mathrm{Se}$ & 0.0931 & 0.999546 \\
\hline $0.3 \mathrm{~mm} \mathrm{a}-S e / 2 \mathrm{~mm} \mathrm{Al}$ & 0.144 & 0.999998 \\
\hline $2 \mathrm{~mm} \mathrm{Al} / 0.5 \mathrm{~mm} \mathrm{a-Se}$ & 0.0274 & 0.999115 \\
\hline $0.5 \mathrm{~mm} \mathrm{a-Se} / 2 \mathrm{~mm} \mathrm{Al}$ & 0.0349 & 0.998159 \\
\hline $1 \mathrm{mmCu} / 0.3 \mathrm{mma}-\mathrm{Se}$ & 0.0954 & 0.999698 \\
\hline $0.3 \mathrm{mma}-\mathrm{Se} / 1 \mathrm{mmCu}$ & 0.126 & 0.999497 \\
\hline
\end{tabular}

with fitting parameter $T_{m}$, and the results of the modelling are summarized in Table 3.1 . The parameters $D$ and $D_{m}$ have been replaced by $T$ and $T_{m}$, respectively because the dose $D$ is proportional to the radiation time $T$.

The relative $\mathrm{x}$-ray sensitivity of the receptor is calculated from

$$
\alpha \propto \frac{1}{T_{m} \cdot(d)^{\frac{1}{3}}}
$$

and is compared to the Monte Carlo results obtained in Chapter 3. Table 3.2 shows the results of three receptors with the same front plate $(2 \mathrm{~mm} \mathrm{Al})$ and different a-Se thicknesses. As expected by the Monte Carlo calculation, x-ray sensitivity increases with the a-Se thickness. The calculated value agrees with the measured one very well at 0.3 
Table 3.2 Relative $x$-ray sensitivity of recentors with a $2 \mathrm{~mm} \mathrm{Al} \mathrm{front} \mathrm{plate.}$

\begin{tabular}{|c|c|c|}
\hline Receptor & Relative o (measured) & Relative o (Monte Carlo) \\
\hline $2 \mathrm{~mm} \mathrm{Al} / 0.15 \mathrm{~mm} \mathrm{a}-\mathrm{Se}$ & 1 & 1 \\
\hline $2 \mathrm{~mm} \mathrm{Al} / 0.3 \mathrm{~mm} \mathrm{a-Se}$ & 2.343 & 2.314 \\
\hline $2 \mathrm{~mm} \mathrm{Al} / 0.5 \mathrm{~mm} \mathrm{a-Se}$ & 6.712 & 4.196 \\
\hline
\end{tabular}

Table 3.3 Relative $x$-ray sensitivity of receptors with different front metal plates.

\begin{tabular}{|c|c|c|}
\hline Receptor & Relative $a$ (measured) & Relative a (Monte Carlo) \\
\hline $2 \mathrm{~mm} \mathrm{Al} / 0.3 \mathrm{~mm} \mathrm{a-Se}$ & 1 & 1 \\
\hline $1 \mathrm{~mm} \mathrm{Cu} / 0.3 \mathrm{mma}-\mathrm{Se}$ & 0.976 & 1.178 \\
\hline
\end{tabular}

$\mathrm{mm}$ but is smaller at $0.5 \mathrm{~mm}$. The effects of a $1 \mathrm{~mm} \mathrm{Cu}$ front plate and a $2 \mathrm{~mm} \mathrm{Al}$ front plate on the $x$-ray sensitivity of a $0.3 \mathrm{~mm}$ a-Se layer are compared in Table 3.3, where discrepancy occurs. The larger enhancement of $x$-ray sensitivity by the $1 \mathrm{~mm} \mathrm{Cu}$ plate was not observed. However, when a $1 \mathrm{~mm} \mathrm{Cu}$ front plate is compared to a back plate (Table 3.4), a good agreement between the calculated and measured data is evident. These cross examinations indicate that $x$-ray sensitivity is primarily dominated by the thickness of the a-Se. A front metal can enhance $\mathrm{x}$-ray sensitivity, but the composition of the front metal plate does not appear to be as important.

\section{$\S \S$ 3.5.3 Polyenergetic Photons}

Most of the photon beams used in radiation treatment are produced with medical 
Table 3.4 Relative $x$-ray sensitivity of a receptor with front and back metal plates.

\begin{tabular}{|c|c|c|}
\hline Receptor & Relative $\alpha$ (measured) & Relative o (Monte Carlo) \\
\hline $0.3 \mathrm{~mm} \mathrm{a-Se} / 1 \mathrm{~mm} \mathrm{Cu}$ & 1 & 1 \\
\hline $1 \mathrm{~mm} \mathrm{Cu} / 0.3 \mathrm{~mm} \mathrm{a-Se}$ & 1.320 & 1.363 \\
\hline
\end{tabular}

linear accelerators. Electrons are accelerated to a very high speed and strike a metal target. The drastic deceleration of these electrons causes bremsstrahlung radiation. Due to the stochastic nature of the interaction between the electrons and the target, the generated $x$ rays do not have the same energy but rather fall in a spectrum. The upper limit of this energy spectrum is at the energy of primary electrons which corresponds to the complete energy transfer from a primary electron to a photon. Because of this spectrum, a therapeutic photon beam is usually characterized by the acselerating potential for the primary electrons.

Receptor sensitivity is also measured on a Clinac $2300 \mathrm{C} / \mathrm{D}$ (Varian Oncology Systems, Palo Alto, Califomia). The Clinac $2300 \mathrm{C} / \mathrm{D}$ can output two photon beams: 6 MV and $23 \mathrm{MV}$. Portal images are usually acquired with the $6 \mathrm{MV}$ beam even though the treatment beam could be different. The receptors were radiated at $179 \mathrm{~cm}$ from the source with the $6 \mathrm{MV}$ beam. The output of the Clinac $2300 \mathrm{C} / \mathrm{D}$ is quantified in terms of Monitor Units (MU), to which the corresponding dose is determined in calibration.

The measured radiation discharging curves of three aluminum plates and copper plate 


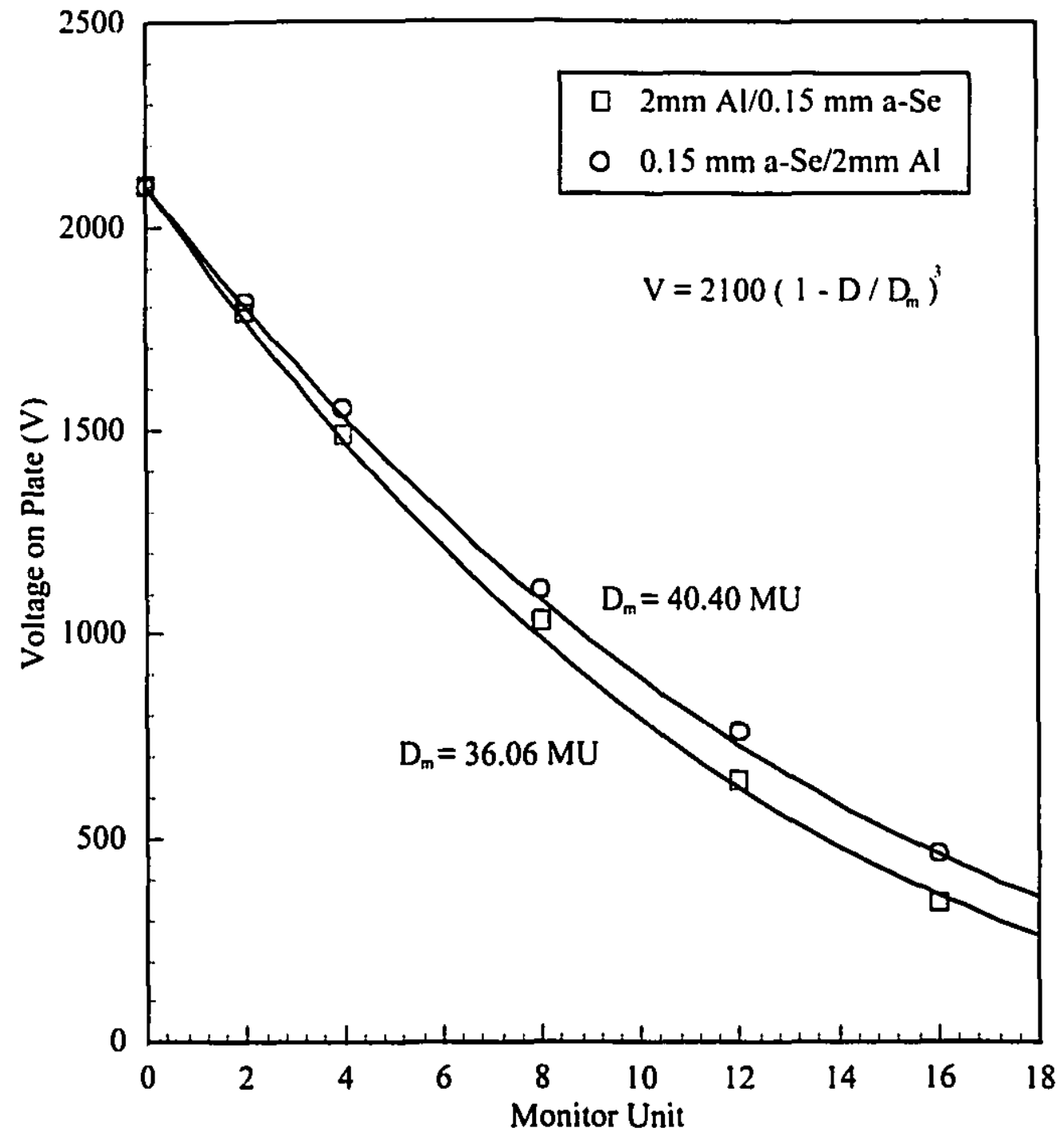

Figure 3.8. Sensitivity curves of a $2 \mathrm{~mm} \mathrm{Al} / 0.15 \mathrm{~mm}$ a-Se receptor measured on a Clinac $2300 \mathrm{C} / \mathrm{D}$ with the front plate and back plate configurations. The dose rate at the receptor is $0.3 \mathrm{cGy} / \mathrm{MU}$. 


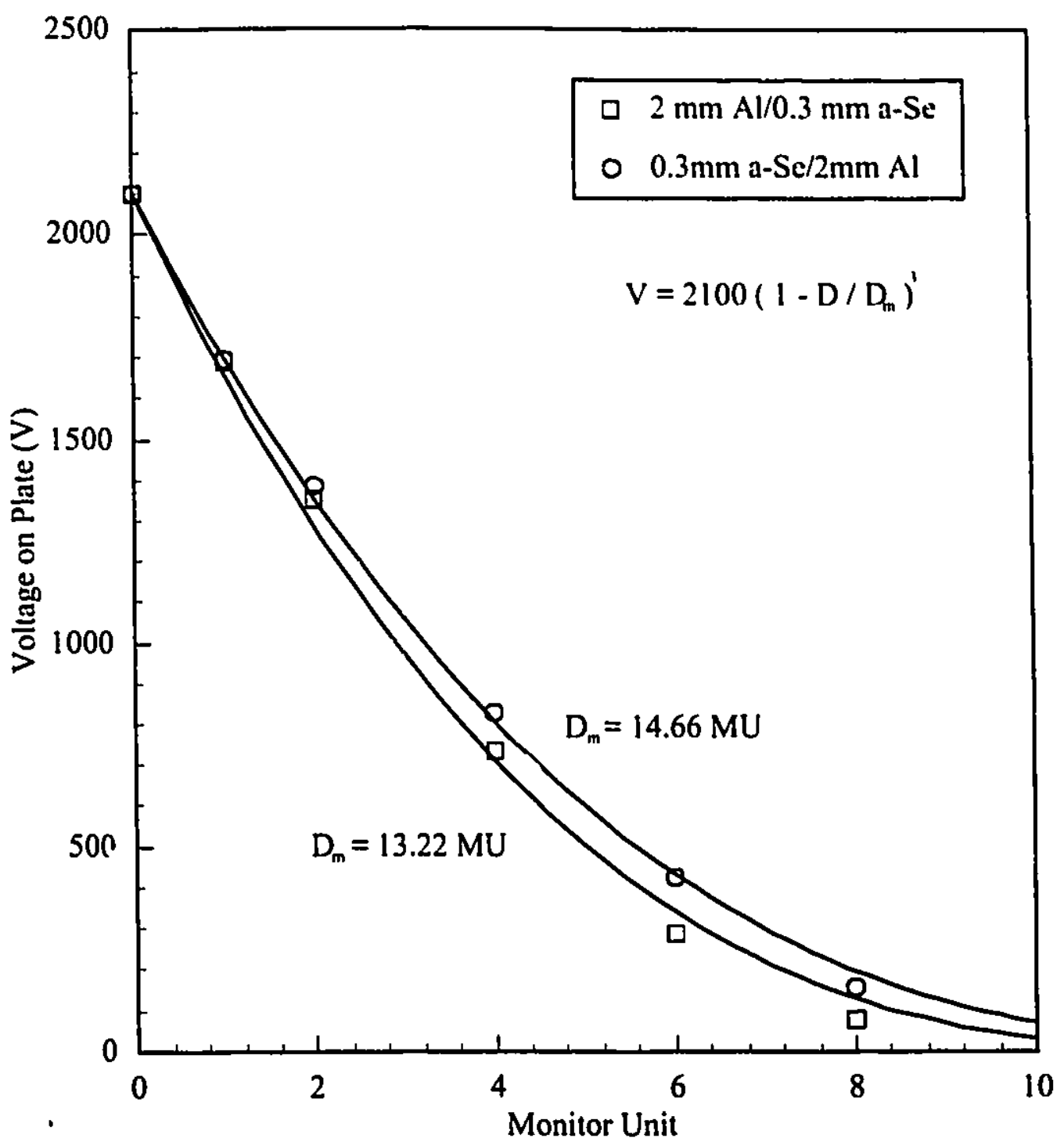

Figure 3.9. Sensitivity curves of a $2 \mathrm{~mm} \mathrm{Al} / 0.3 \mathrm{~mm}$ a-Se receptor measured on a Clinac $2300 \mathrm{C} / \mathrm{D}$ with the front plate and back plate configurations. The dose rate at the receptor is $0.3 \mathrm{cGy} / \mathrm{MU}$. 


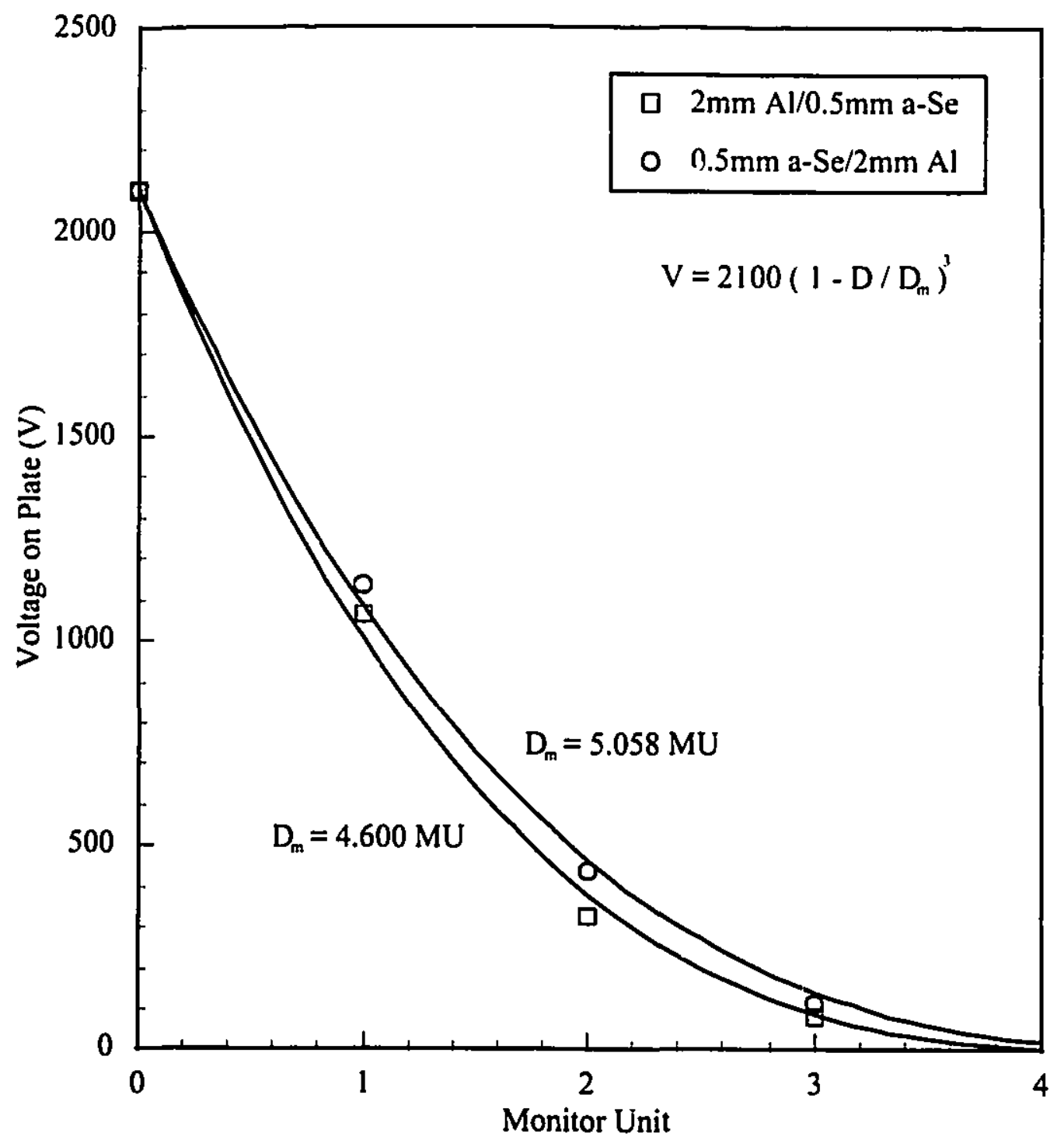

Figure 3.10. Sensitivity curves of a $2 \mathrm{~mm} \mathrm{Al} / 0.5 \mathrm{~mm}$ a-Se receptor measured on a Clinac $2300 \mathrm{C} / \mathrm{D}$ with the front plate and back plate configurations. The dose rate at the receptor is $0.3 \mathrm{cGy} / \mathrm{MU}$. 


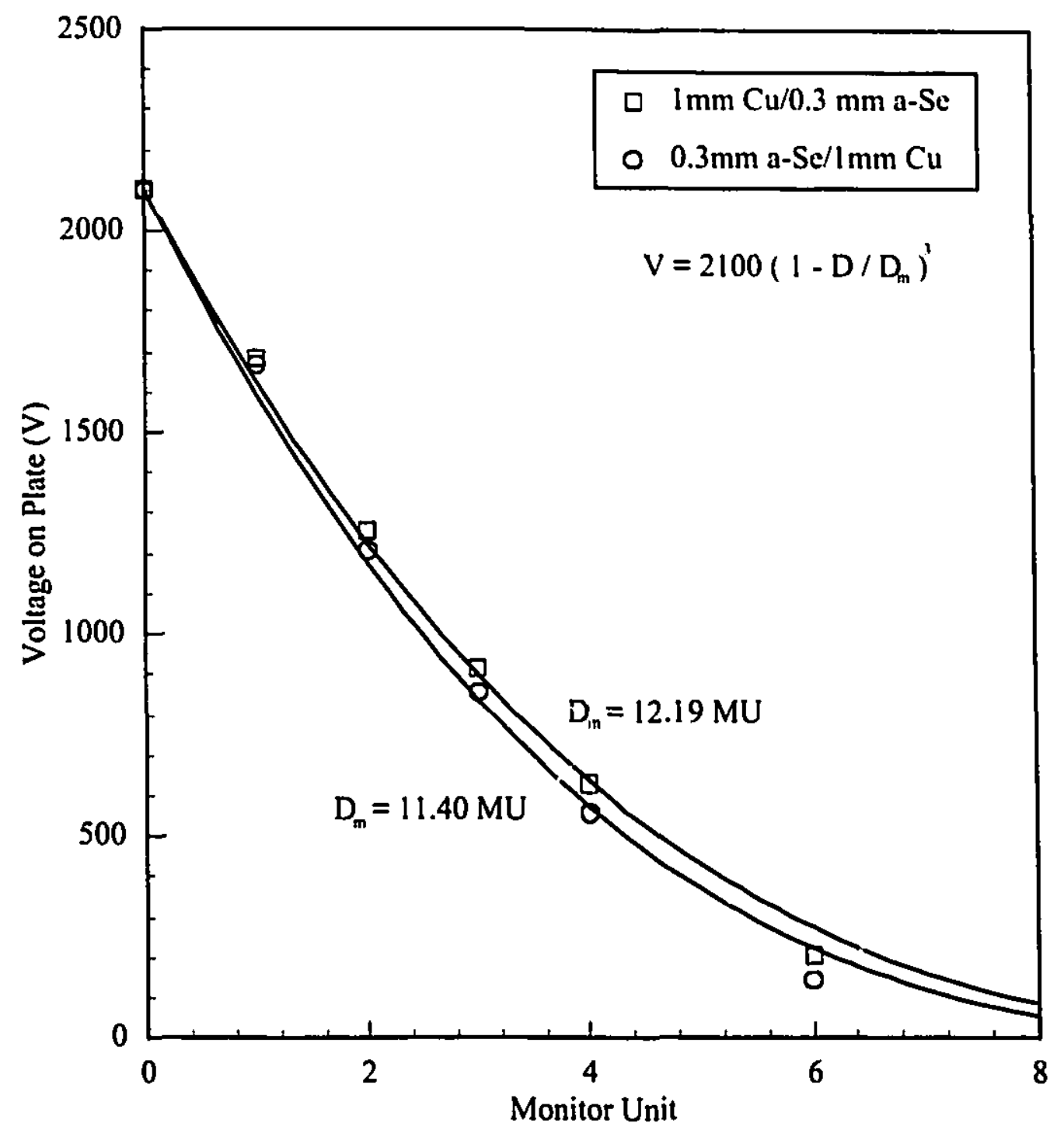

Figure 3.11. Sensitivity curves of a $1 \mathrm{~mm} \mathrm{Cu} / 0.3 \mathrm{~mm}$ a-Se receptor measured on a Clinac $2300 \mathrm{C} / \mathrm{D}$ with the front plate and back plate configurations. The dose rate at the receptor is $0.3 \mathrm{cGy} / \mathrm{MU}$. 


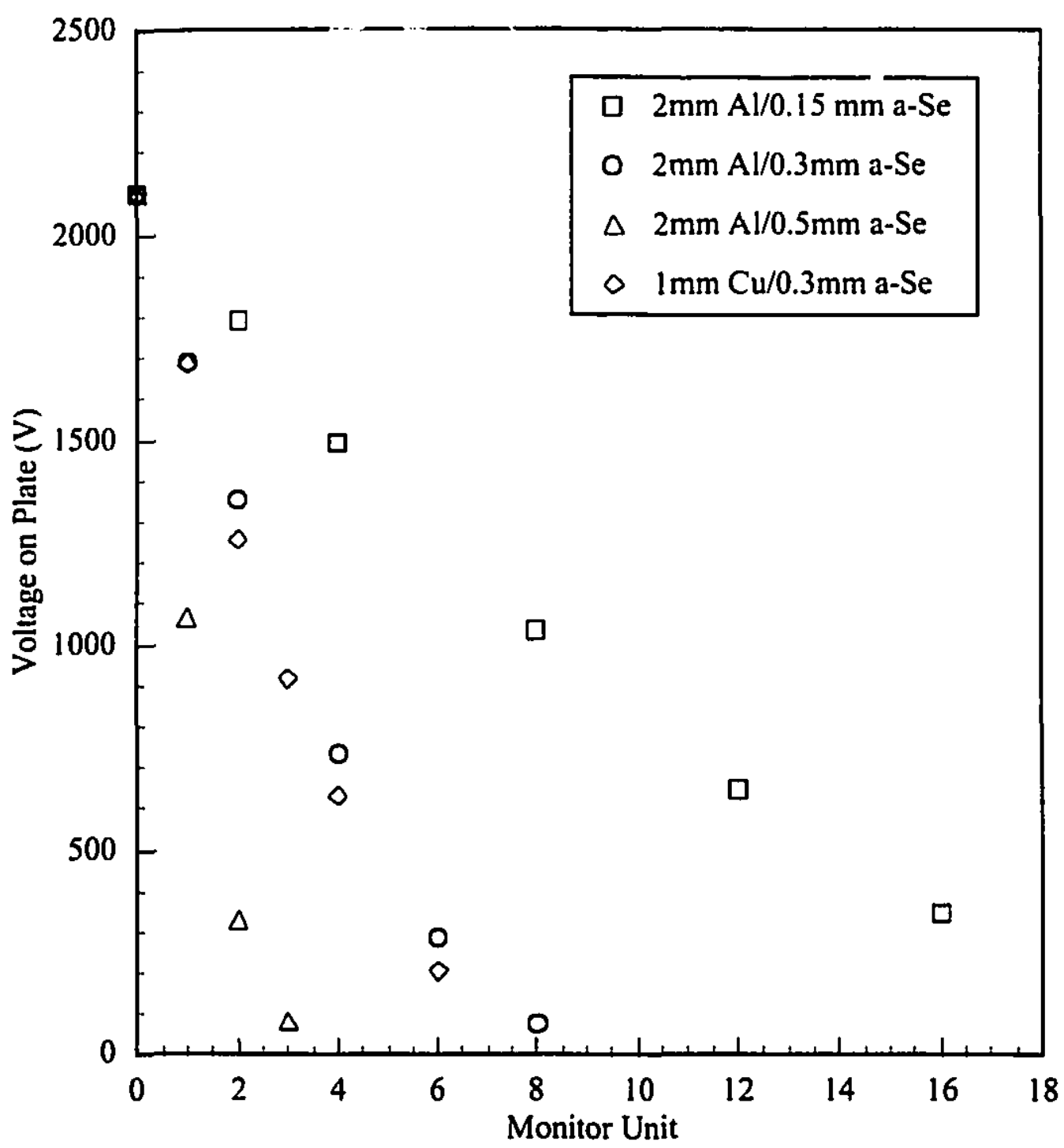

Figure 3.12. The sensitivity curves of four different a-Se receptors measured on a Clinac $2300 \mathrm{C} / \mathrm{D}$. The dose rate at the receptor is $0.3 \mathrm{cGy} / \mathrm{MU}$. 
are shown individually in Figure 3.8 to Figure 3.11, and together in Figure 3.12. Error bars are not plotted because they are too small. No significant difference can be observed between front plate orientation and back plate orientation. The reason is that the smaller weights of the low energy components are compensated by the high absorption efficiency at low energies when the a-Se is directly exposed. A front plate, on the other hand, can enhance the absorption of the high energy components but at the same time attenuates the low energy photons. Once again, the thickness of the a-Se layer appears to dominate in the discharging process. As discussed previously, receptor sensitivity can only be characterized in a relative manner. The dose rate (to a small mass of tissue) at the position of the receptor $(179 \mathrm{~cm}$ from the source) is $0.3 \mathrm{cGy} / \mathrm{MU}$.

Similar modelling is done with

$$
V(D)=V_{0}\left[1-\frac{D}{D_{m}}\right]^{3}
$$

where $D$ is the amount of radiation in monitor units and, correspondingly, $D_{m}$ is the amount of radiation needed to completely discharge the receptor. The results are listed in Table 3.5.

Judging by the correlation coefficient of the curve fitting, the radiation discharging model seems to work very well even with polyenergetic photons. The sensitivity is again dominated by the a-Se thickness. A thick a-Se layer leads to a large contrast but small dynamic range due to its high energy absorption. A slight acceleration of discharging by 
Table 3.5 Monitor units required for complete discharge of different receptors on Clinac $2300 \mathrm{C} / \mathrm{D}$ and correlation coefficients obtained from the modelling

\begin{tabular}{|c|c|c|}
\hline Receptor & $D_{m}(M L i)$ & $R$ \\
\hline $2 \mathrm{~mm} \mathrm{Al} / 0.15 \mathrm{~mm} \mathrm{a-Se}$ & 36.06 & 0.99931697 \\
\hline $0.15 \mathrm{~mm} \mathrm{a}-\mathrm{Se} / 2 \mathrm{~mm} \mathrm{Al}$ & 40.40 & 0.99946616 \\
\hline $2 \mathrm{~mm} \mathrm{Al} / 0.3 \mathrm{~mm} \mathrm{a-Se}$ & 13.22 & 0.99883452 \\
\hline $0.3 \mathrm{~mm} \mathrm{a-Se} / 2 \mathrm{~mm} \mathrm{Al}$ & 14.66 & 0.99955136 \\
\hline $2 \mathrm{~mm} \mathrm{Al} / 0.5 \mathrm{~mm} \mathrm{a-Se}$ & 4.600 & 0.99891336 \\
\hline $0.5 \mathrm{~mm} \mathrm{a}-\mathrm{Se} / 2 \mathrm{~mm} \mathrm{Al}$ & 5.058 & 0.99928159 \\
\hline $1 \mathrm{~mm} \mathrm{Cu} / 0.3 \mathrm{~mm} \mathrm{a-Se}$ & 12.19 & 0.99880869 \\
\hline $0.3 \mathrm{~mm} \mathrm{a-Se} / 1 \mathrm{~mm} \mathrm{Cu}$ & 11.40 & 0.99850578 \\
\hline
\end{tabular}

the $2 \mathrm{~mm} \mathrm{Al} \mathrm{front} \mathrm{plate} \mathrm{can} \mathrm{be} \mathrm{observed} \mathrm{while} \mathrm{the} 1 \mathrm{~mm} \mathrm{Cu}$ front plate seems to reduce $\mathrm{x}$ ray sensitivity slightly. This is probably because the $\mathrm{Cu}$ plate acts more like an attenuator than a build-up layer to the low energy components of the primary $x$-ray beam. At low energies, photoelectric effect is the dominant interaction and the corresponding attenuation coefficient increases drastically as the atomic number increases. ${ }^{10}$ Even though the back $\mathrm{Cu}$ plate leads to a slightly higher sensitivity than the front plate, it cannot be used for acquiring portal images as will be demonstrated in the next section.

\section{§ 3.6 Phantom Tests}

Primarily determined by the receptor sensitivity, image contrast is also affected by 
the spatial resolution and noise level of the receptor. Receptor blur and noise will reduce contrast. "Systematic evaluation of the performance of an imager requires measuring not only the sensitivity but also the modulation transfer function and the detective quantum efficiency. These measurements are not ready at this stage of the development for the following reasons.

The MTF is usually measured with the line spread function method where the receptor is exposed to a very narrow parallel beam and a profile is obtained by scanning the receptor across the line image. ${ }^{12}$ The module of the Fourier transform of the line spread function is the modulation transfer function. Although conceptually straightforward, this measurement cannot be carried out without equipment that can provide mechanical motions with high precision while supporting heavy beam-shaping blocks.

Measuring detective quantum efficiency requires the measuring noise power spectrum. It can be done by radiating the receptor with an open field, scanning the image, and then taking the Fourier transform to calculate the noise power spectrum. The condition of this measurement is that the receptor must have a high degree of uniformity. This requirement cannot be satisfied by the plates used in this preliminary study.

Phantom test is an alternative. It is simple to perform and the results can be compared with those from commercial systems. The disadvantage is that the individual properties of the imager can not be isolated. A contrast-detail phantom has been developed by Munro 
et $a l^{13}$ for testing portal imagers and has become commercially available (Radiation Measurements, Inc., Middleton, Wisconsin). It is a $13 \mathrm{~mm}$ thick $25 \times 25 \times 1.3 \mathrm{~cm}^{3}$ aluminum slab with a $10 \times 10$ array of holes with a decreasing depth in each row and a decreasing size in each column (Table 3.6). Spaced at a center to center distance of $18.2 \mathrm{~cm}$, these holes can provide contrast variation at different spatial detail levels. Systematic tests with this phantom of a commercial EPID have been reported by Dong and Boyer. ${ }^{14}$ Our prototype imager is still in the development stage, and it will impose limitations on systematic phantom tests. For example, the scanning area is smaller than the contrast detail phantom; the image receptors have defects. Due to these reasons, we only conducted visual inspection of images of the contrast-detail phantom.

The contrast-detail phantom was imaged on the Clinac $2300 \mathrm{C} / \mathrm{D}$ under the same conditions as in the sensitivity measurements with the phantom at $2 \mathrm{~cm}$ above the receptor. Figure 3.13 shows the images obtained with a Cu/a-Se plate receptor with voth the front plate and the back plate configurations. The better visibility of the holes in the front plate image clearly demonstrates the scatter removal function of the front metal plate. Because of the high sensitivity of a-Se to low energy photons, the scatter from the phantom completely obscures the image in the back plate configuration.

Images of the same phantom were also acquired with a commercial fluoroscopic EPID and a matrix ionization chamber EPID for comparison (Figure 3.15). While having 
Table 3.6 The sizes and depths of the holes on the contrast-detail phantom.

\begin{tabular}{|c|c|c|c|}
\hline Row & Column & Diameter $(\mathrm{mm})$ & Depth $(\mathrm{mm})$ \\
\hline 1 & 1 & 12.80 & 4.57 \\
\hline 2 & 2 & 11,10 & 3.23 \\
\hline 3 & 3 & 7.93 & 2.29 \\
\hline 4 & 4 & 5.56 & 1.63 \\
\hline 5 & 5 & 4.76 & 1.14 \\
\hline 6 & 6 & 3.97 & 0.76 \\
\hline 7 & 7 & 3.18 & 0.51 \\
\hline 8 & 8 & 2.38 & 0.43 \\
\hline 9 & 9 & 1.59 & 0.36 \\
\hline 10 & 10 & 1.19 & 0.29 \\
\hline
\end{tabular}

a much higher quality, our image was acquired with only 2 MUs at a distance of $179 \mathrm{~cm}$ from the source. The fluoroscopic EPID and the matrix ionization chamber required 50 MUs and 23 MUs, respectively, at $140 \mathrm{~cm}$ from the source. An image of a head phantom is also shown in Figure 3.15 to demonstrate the potential of the prototype imager.

\section{§ 3.7 Conclusions}

We have measured the radiation discharging curves of a-Se image receptors with different sensitive volumes and different build-up metal plates. The sensitivity of these receptors to therapy photons was found to be quite high and to be dominated by the 


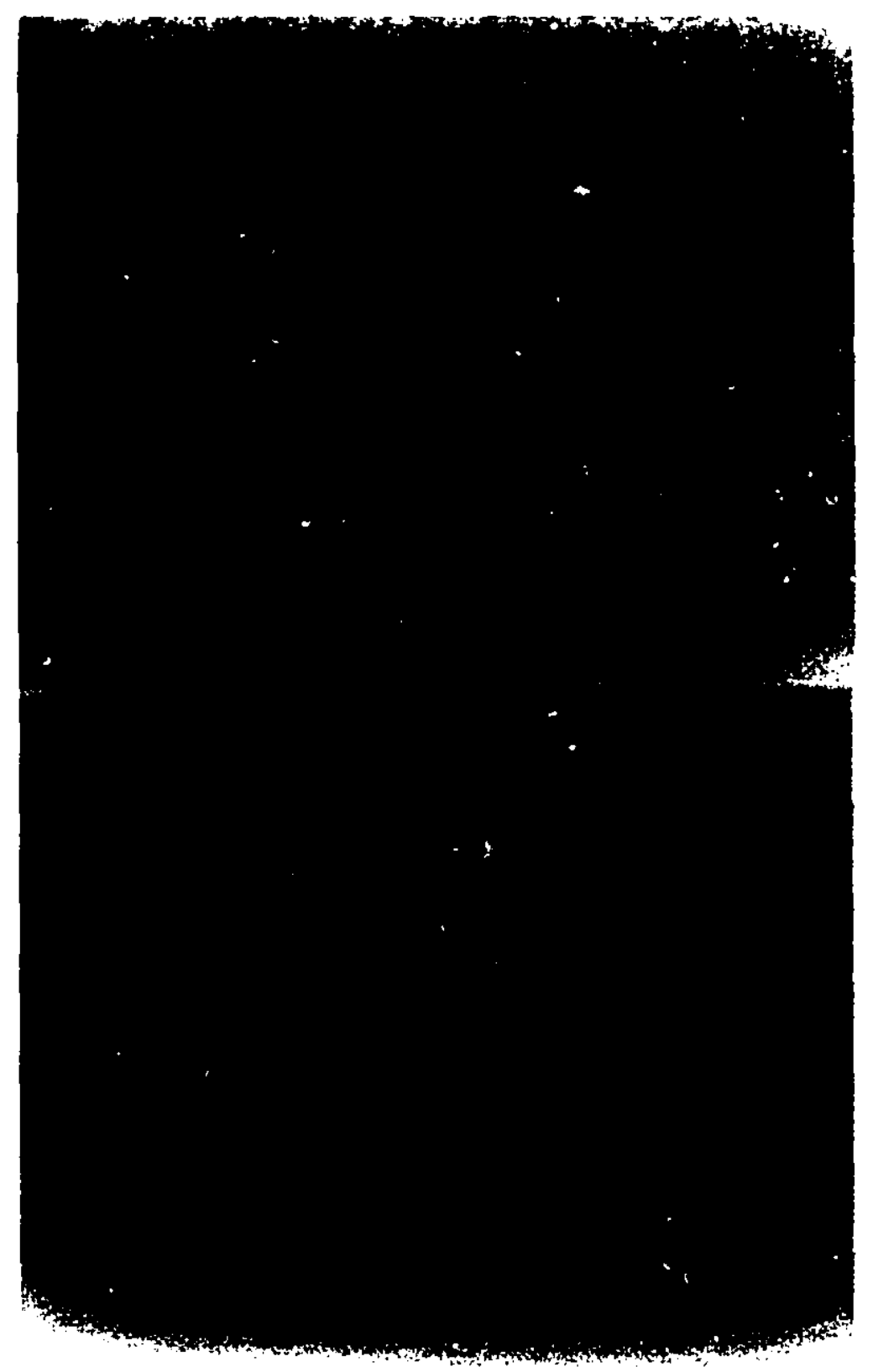

Figure 3.13. Images of a contrast-detail phantom acquired with a $1 \mathrm{~mm} \mathrm{Cu} / 300 \mu \mathrm{m}$ a-Se receptor on a Clinac $2300 \mathrm{C} / \mathrm{D}$ linear accelerator by using the $6 \mathrm{MV}$ beam. Top: front plate image; bottom: back plate image. 


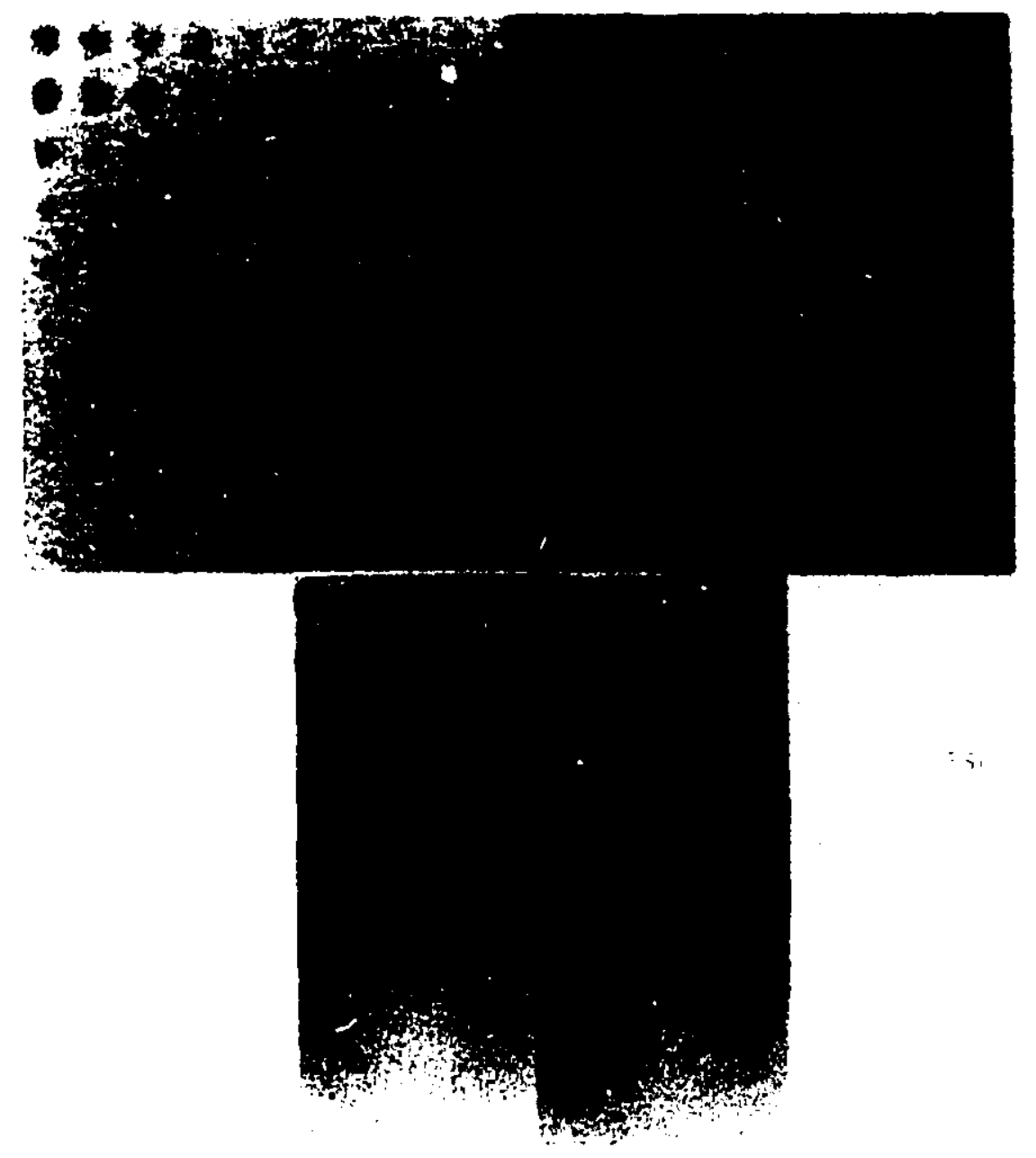

Figure 3.14. Images of a contrast-detail phantom acquired with a fluoroscopic EPID (top left), a matrix ionization chamber EPID (top right) and our prototype imager (bottom) on a Clinac $2300 \mathrm{C} / \mathrm{D}$ linear accelerator by using the $6 \mathrm{MV}$ beam (Courtesy of Tony Falco). 


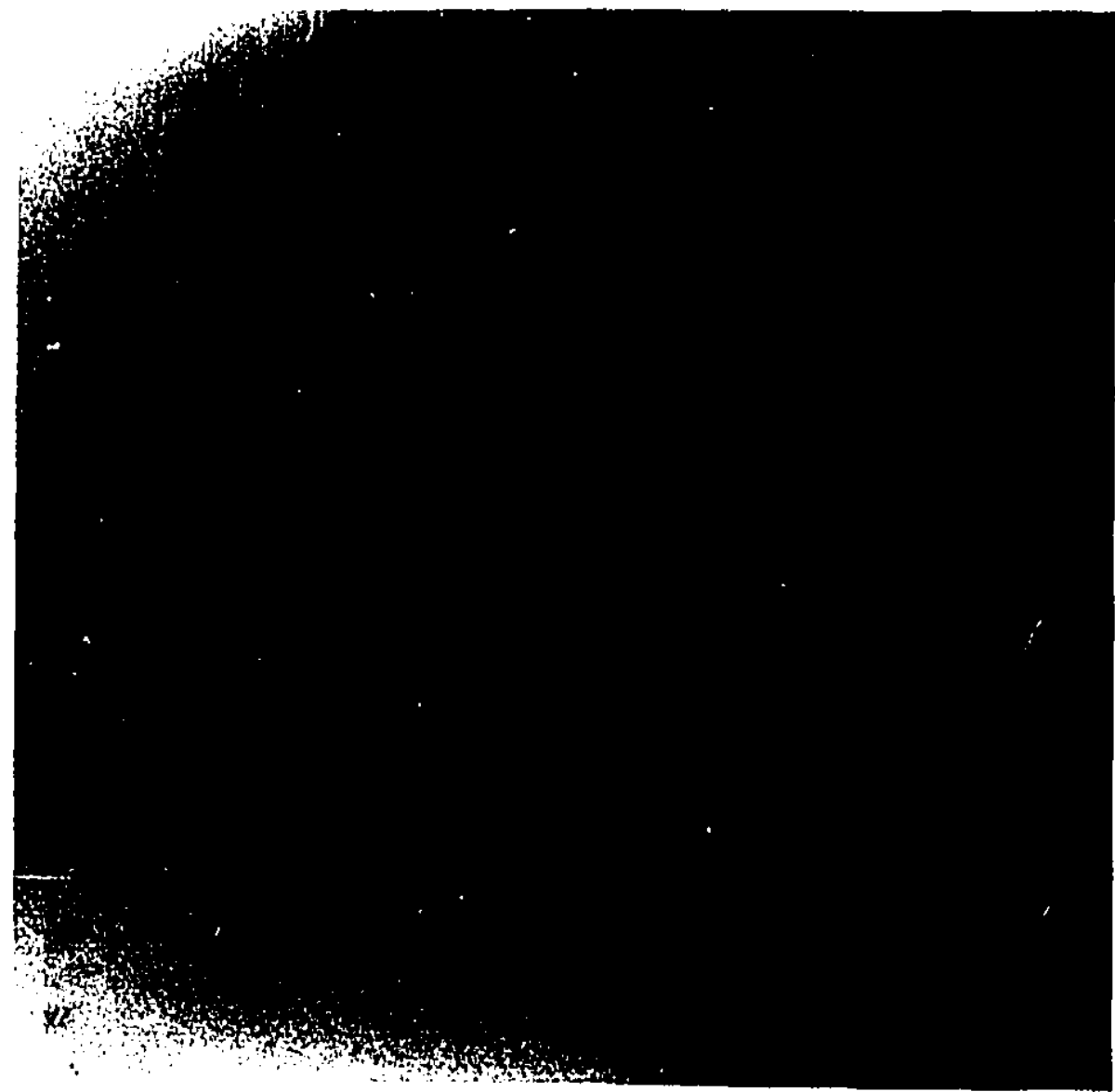

Figure 3.15. Image of a head phantom acquired with a $1.5 \mathrm{~mm} \mathrm{Cu} / 300 \mu \mathrm{m}$ a-Se receptor on a Clinac $2300 \mathrm{C} / \mathrm{D}$ linear accelerator by using the $6 \mathrm{MV}$ beam (Courtesy of Tony Falco). 
a-Se thickness. A front metal plate can enhance the energy absorption at high $\mathrm{x}$-ray energies while the composition of the metal plate appears not to be important. The front metal plate is also required for removing scattered radiation from the object which could degrade and even completely destroy the image (Figure 3.13). Image cortrast and spatial resolution were found to be comparable to those of commercial fluoroscopic portal imagers. Preliminary contrast-detail studies also confirmed that the noise level is very low in electrostatic imaging due the high collection efficiency of charge carriers. The performance of the prototype imager indicates that significant reduction of dose currently required for taking portal images can be expected with a-Se receptors. 


\section{References}

I J. W. Boag, "Xeroradiography," Physics in Medicine and Biology 18, 3-37 (1973).

${ }^{2}$ L. Wolfe, L. Kalisher, and B. Considine, "Cobalt-60 treatment tield verification by xeroradiography," American Journal of Roentgenology 118, 916-918 (1973)

${ }^{3}$ I. Brodie and R. A. Gutcheck, "Minimum exposure estimates for information recording in diagnostic radiology," Medical Physics12, 362-367 (1985).

${ }^{4}$ A. Zermeno, T. Kirby, R. Cowart, L. Marsh, and P. Ong, "Laser readout of electrostatic images," Proceedings of SPIE 173, $81-87$ (1979).

${ }^{5}$ E. L. Cook, J. D. Edwards, O. L. Nelson and J. E. Potts, "Performance of a high resolution radiographic detector," inThe society of Imaging Science and Technology 47th Annual Conference ICPS, 669 (1994).

6 J. A. Rowlands and D. M. Hunter, "X-ray imaging using amorphous selenium: Photoinduced discharge (PID) readout for digital general radiography," Medical Physics22, 1983-2005 (1995).

7 I. Brodie and R. A. Gutcheck, "Radiographic information theory and application to mammography," Medical Physics 9, 79-94 (1982).

${ }^{8}$ U. Neitzel, I. Maack, and S. Gunther-Kohfahl, "Image quality of a digital chest radiography system based on a selenium detector," Medical Physics 21, 509-516 (1994). 
9 J. A. Rowlands, G. DeCrescenzo and N. Araj, "X-ray imaging using amorphous selenium: Determination of $\mathrm{x}$-ray sensitivity by pulse height spectroscopy," Medical Physics19, 1065-1069 (1992).

10 H. E. Johns and J. R. Cunningham, The Physics of Radiology( 4th edition, Springfield, Illinois 1983).

II P. Sprawls, Physical principles of medical imaging (Aspen, Gaithersburg, Maryland 1993), 2nd edition.

12 R. Shaw, "Some fundamental properties of xeroradiographic images," SPIE Proceedings 70, 359-363 (1975).

13 P. Munro, J. A. Rawlinson, and A. Fenster, "Therapy Imaging: A signal-to-noise analysis of fluoroscopic imaging systems for radiotherapy localization," Medical Physics 17, 763-772 (1990).

14 L. Dong and A. L. Boyer, "An objective method for evaluating electronic portal imaging devices," Medical Physics 21, 755-760 (1994). 


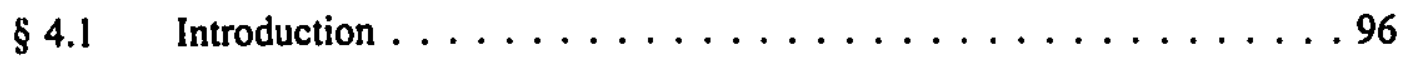

$\S 8.1 .1 \quad$ Objectives $\ldots \ldots \ldots \ldots \ldots \ldots \ldots \ldots \ldots \ldots$

$\$ \$ 4.1 .2 \quad$ Scope $\ldots \ldots \ldots \ldots \ldots \ldots \ldots \ldots \ldots$

$\S 4.2$ Materials and Methods $\ldots \ldots \ldots \ldots \ldots \ldots \ldots$

$\S \S 4.2 .1 \quad$ Image Processing System $\ldots \ldots \ldots \ldots \ldots \ldots$

$\S$ 4.2.2 Image Acquisition $\ldots \ldots \ldots \ldots \ldots \ldots \ldots \ldots$

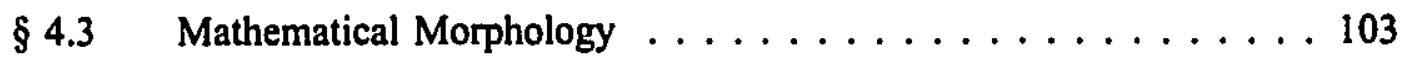

$\S \S 4.3 .1 \quad$ Fundamental Operations . . . . . . . . . . . . . 104

$\S \S 4.3 .2$ Complex Operations and Algorithms . . . . . . . 108

$\S \S$ 4.3.3 Digital Morphology . . . . . . . . . . . . 111

$\S \S 4.3 .4 \quad$ Gray Scale Morphology . . . . . . . . . . . . . 114

$\S \S$ 4.3.5 Morphological Gradient $\ldots \ldots \ldots \ldots \ldots \ldots \ldots$

$\S 4.4 \quad$ Edge Detection $\ldots \ldots \ldots \ldots \ldots \ldots \ldots \ldots \ldots \ldots$

$\S \S 4.4$ Gradient Operators $\ldots \ldots \ldots \ldots \ldots \ldots \ldots \ldots$

$\S \S 4.4 .2 \quad$ Optimal Edge Detectors $\ldots \ldots \ldots \ldots \ldots \ldots \ldots$

$\S \S 4.4 .3 \quad$ Morphological Edge Detectors $\ldots \ldots \ldots \ldots \ldots \ldots$ 
Chapter 4

Segmentation of Portal Images

$\S 4.5 \quad$ Algorithm Development . . . . . . . . . . . . . . . . . . . . 124

$\S \$ 4.5 .1 \quad$ Basic Approach $\ldots \ldots \ldots \ldots . \ldots \ldots$

$\S \S 4.5 .2 \quad$ Feature Extraction $\ldots \ldots \ldots \ldots$. . . . . . . 130

$\S \S 4.5 .3 \quad$ Edge Localization . . . . . . . . . . . . . . . . . 130

$\S \S 4.5 .4 \quad$ Automation of Segmentation $\ldots \ldots \ldots$. . . . . 138

$\S 4.6 \quad$ Discussions $\ldots \ldots \ldots \ldots \ldots \ldots \ldots$

95 


\section{$\$ 4.1$ Introduction}

\section{$\$ \$$ 4.1.1 Objectives}

The goal of portal imaging is to ensure accurate execution of a treatment plan, specifically, to reduce errors introduced in the radiation dose delivery procedure. Errors in a treatment are determined by how closely the radiation beam is shaped to the prescription and how accurately the shaped beam is localized to the target. With portal images, these errors can be detected and corrected before and during the treatment. The first step of error detection is to accurately delineate and characterize the radiated area in a portal image. With the advent of electronic portal imaging devices, not only can portal images be acquired digitally, they can also be analyzed with digital processing techniques. Automatic and accurate extraction of the treatment field from digital portal images acquired with EPIDs has been investigated recently. Bijhold et al developed a segmentation algorithm based on local gradient of grayscale variation in portal images. In this algorithm, a rough edge of the radiation field was obtained from global thresholding and then modified to the contour of maximum local gradient points. Another algorithm employing an optimal edge detector and maximum local gradient tracing was reported by Leszczynski et al. $^{2}$

Even though EPIDs are becoming more and more popular, the radiographic film still remains the major means of acquiring portal images. Portal films can be digitized and 
the digital images can be analyzed with computer algorithms. The results of off-line analysis can be used to adjust the next treatment session accordingly. Portal films post a more difficult task for image segmentation algorithms because of the difference in image acquisition. A portal film is usually exposed twice: first with the shaped beam and then with an open field. This double-exposure technique shows not only the shape of the field but also the location of the beam with respect to the surrounding anatomy. ${ }^{3}$ As a result, a transparent shadow of the radiation field is overlaid on the background anatomy making the double-exposure image very difficult to segment. On an EPID, however, two images are acquired with the shaped beam and an open field separately and the two singleexposure images are overlaid on top of each other. The radiation field can be delineated from the single-exposure image acquired with the shaped beam where the region outside the radiation field is uniform. In this chapter, a robust algorithm will be presented for automatic extraction of the radiation field from double-exposure portal images.

Another purpose of portal image segmentation is to enhance image contrast for better visualization of anatomical details. As discussed in Chapter 1, portal images have an inherently low contrast due to the high energy of the x-ray beam. Contrast enhancement can be achieved with different kinds of histogram-based transformations, among which histogram equalization is a very popular one. The histogram of an image is a plot of number of pixels versus pixel value, i.e., the occurrence of pixels at each gray level. 
For a low contrast image, its histogram occupies a small region of the whole display range. Gray level variation corresponding to the content of the image is small. The histogram equalization process transforms the value of each pixel such that the histogram of the transformed image is uniform across the whole display range, i.e. to assign equal number of pixels to each display level to achieve the optimum visualization. If histogram equalization is simply applied to a double-exposure portal image, its effect is limited because the radiation field shadow introduces an additional peak in the lower part of the histogram making the histogram not being sufficiently stretched. This problem can be solved by applying contrast enhancement to different regions separately. A selective histogram equalization approach has been proposed by Cronks and Fallone $e^{4-6}$ for enhancing the contrast of double-exposure portals. The objective of this chapter is to improve the robustness of the segmentation required by this approach.

\section{$\$ \S 4.1 .2$ Scope}

The previous two chapters are concerned with image formation and acquisition, the work presented in this chapter falls in another category: postprocessing. Section 4.2 describes the image processing system, followed by a brief review of some basic concepts in mathematical morphology (Section 4.3) and edge detection (Section 4.4) which serve as the foundation of this chapter. The development of a robust algorithm for automatic segmentation of portal images will be presented in Section 4.5. 


\section{$\S$ 4.2 Materials and Methods}

\section{§ 4.2.1 Image Processing System}

An imaging system having hardware processing ability has been employed in this study. It consists of a VDC3874 video camera (Sanyo Electric, Inc., Japan) with a user designed light box, a FD-2000 laser film digitizer (Du Pont, U.S.A.), a 386 PC (MaxSys, Inc. U.S.A.), a Matrox Image-Series IM-1280 imaging board set (Matrox Electronic Systems, Canada), and a Mitsubishi HL6905 Diamond Scan 19' (Mitsubishi Electric Corp., Japan) high resolution image monitor. A workstation (IRIS ELAN4000, SiliconGraphics, Mountainview, Califomia) was added to the system later as a processing unit parallel to the IM-1280 imaging board set.

Image-Series. As the central part of this system, the Image-Series performs image digitization and processing and controls image display on the image monitor. It is an intelligent board set consisting of a base board (IM-1280), a real time processor board (RTP) and a digitizer board. The three boards are connected by an image bus.

Base board. The Base Board is the central board of the Image-Series. It has a Graphics System Processor (GSP) which controls the whole Image Series. The GSP receives opcodes transmitted from the Host computer, decodes them, and then either sets up the appropriate hardware or executes the request itself. The base board also hosts buffers 
which feed data to the on-board video display controller and serve as the main storage area for images, processing and graphics. The frame buffers are organized to provide very fast data transfers between buffers and all other Image-Series boards (up to 30 million pixeis per second).

Image-RTP. Consisting of a Data Formatter, a Data-Router, a Cascaded ALU, a Neighborhood Processor and a Statistical Processor, the Image-RTP makes it possible to perform several operations in one $15 \mathrm{MHz}$ pass. It can perform $3 \times 3$ gray scale and $18 \times 16$ binary neighborhood operations in one pass with the Neighborhood Processor, and combine information from two sources with the Cascaded ALU. A data router allows for various data paths through the processing elements.

The Data Formatters, situated on both Image-RTP inputs, convert incoming frame buffer/digitizer data from various input types into the internal 16-bit representation used by the processing pipeline. An on-board Data Router directs data to the appropriate processing elements. It allows results from one processing element to undergo further processing through other processing elements without having to store and then reread the results. The cascaded ALU performs a variety of arithmetic and logical operations. It can preprocess the input or postprocess the output of other processing elements. The neighborhood Processor can perform up to $18 \times 16$ binary and up to 63 gray scale neighborhood and morphological operations in one frame time. It supports 
both rectangular and hexagonal lattices in hardware. The statistical processor has an event counter/comparator, a minimum/maximum comparator, and histogram and profile generator. This prosessor uses Statistical LUT as a storage area for results. The Statistical Processor can use object labels to sort the results of basic feature extraction.

Digitizer Board. The image-ASD supports both analog and digital input, accepts a wide range of source frequencies, has a programmable synchronization generator, and can accept trigger pulses for mono-shot cameras. It supports black and white video sources. or video sources with switchable filters or RGB input on three different channels. This digitizer can only send one analog color component at a time to the Image-Bus. An on-board Digitizer LUT maps digitized data.

Image-Bus. The Image Bus serves as a $30 \mathrm{MHz}$ communication link between the base board and the other Image-Series boards. It is a wide dual-bus interface consisting of an I/O bus for system control, and a processing bus with two 32-bit data paths for high-speed image processing data transfers. The two processing data paths provide simultaneous data flow both to and from data storage.

Host and I/O. The MaxSys 386 PC hosts the Image Series. To a large extent, it is only used as a controller. The VDC 3874 video camera has $\frac{2}{3}$ " CCD array of $800 \times 490$ elements. It uses a $25 \mathrm{~mm}$ lens to focus the image on the CCD. Video output of the 
camera are sent to the Image-Series and digitized by the digitizer board. Films are also digitized with the laser film digitizer (which digitizes a film into $844 \times 1021$ pixels with a depth of 14 bits or $1688 \times 2042$ pixels with a depth of 12 bits. Digital images are displayed on the image monitor which has a resolution of $1280 \times 1024$ pixels.

Software. The Image-Series comes with a complete set of control, processing and graphics modules. It also has a command interpreter that allow the user to issue commands directly to the hardware, but this is performed on a very low level and is difficult to use. Noesis Visilog image processing software has been used as the userboard interface. Visilog is an image processing and analysis software package working with industry-standard Graphics User Interface. It can automatically make use of the abilities of special imaging hardwares. The PC version is developed in the MS-Windows 3.0 or higher environment, and is therefore, particularly suitable for investigations for algorithm development. It also has a library of image analysis, processing and graphics functions for user application development.

\section{§§ 4.2.2 Image Acquisition}

Portal and simulator image pairs are selected randomly from the patient files in Radiation Oncology in the Montreal General Hospital and the Jewish General Hospital. Simulator films are obtained from an AECL Therasim-750 simulator, and portal films are obtained from the following therapy machines: Theratron-780 Co-60 unit ( Atomic 
Energy of Canada), 7 herapi-4 4 MV (SHM Nuclear Systems), EMI-6 6 MV (EMI Therapy Systems) and Clinac-18 10MV (Varian Associates) linear accelerators. The films cover a variety of treatment sites. Since portal films have low spatial resolution and low contrast, they are digitized with the video camera into $256 \times 240$ pixels, each pixel with a depth of 8 bits. Films are also digitized with the FD-200 laser digitizer, and the image file is presently imported into the host of the imaging system via a floppy disk. The standard resolution mode $(844 \times 1024 \times 14$ bits) has been used. Since the image format of Visilog is that the depth of a pixels must be a multiple of a byte, images acquired with the laser digitizer are shrunk into 8 bits deep. An improvement may be achieved by taking advantage of the full 14 bit image, but the advantages may be marginal due to the inherently low contrast features of portal images, therefore we used the video camera for digitization most of the time.

\section{§ 4.3 Mathematical Morphology}

Morphology as a methodology in image processing was introduced by G. Matheron in the $1970 \mathrm{~s}^{7}$ Based on set theory, morphology deals with geometric structures inherent to an image..$^{8-12}$ Geometric information in an image is analyzed by fitting some predefined small geometric shapes, called structuring element, into the image. As a probe, the structuring element is passed over the domain of an image while a set operation is applied around the neighborhood of each element of the image. The geometric information which 
is extracted depends on the operation applied when the structuring element is passed over the image. Since most of the morphological operations used in this study are applied on binary images, we will commence our discussion of morphology in the Euclidean plane. Two-valued images can be considered as sets of points in the Euclidean plane.

\section{$\S \S$ 4.3.1 Fundamental Operations}

As discussed, mathematical morphology is based on set theory. Morphological operations are built upon set operations which are primitive to the morphology level. Besides the usual set operations, union and intersection, another primitive operation, translation, has to be introduced in order to define the basic morphological operations. For a set of points $A$ in the Euclidean plane $R^{2}$, the translation of $A$ by a point $x$ in $R^{2}$ is given by

$$
A+x=\{a+x: a \in A\}
$$

Now we can define the two fundamental operations in morphology, Minkowski addition and subtraction. Given two sets $A$ and $B$ in $R^{2}$, the Minkowski addition (represented by $\oplus)$ of $A$ and $B$ is the union of all the translates of $A$ by each element of $B$,

$$
A \oplus B=\bigcup_{b \in B}(A+b)
$$

while the Minkowski subtraction (represented by $\Theta$ ) is the intersection of all the translates 
of $A$ by each element of $B$,

$$
A \ominus B=\bigcap_{b \in B}(A+b)
$$

where $b$ is an arbitrary element of $B$. Traditionally, morphology was developed in a graphical way. Basically, a small probe is applied to every element of an image, and the manner in which this probe fits within the image is investigated. Based on this strategy, two basic morphological operations can be defined from Minkowski addition and Minkowski subtraction. It can be proven that the Minkowski addition, $A \oplus B$, is equivalent to the union of all the translates of $B$ by each element of $A$ :

$$
\begin{aligned}
A \oplus B & =\bigcup_{y \in B}(A+y) \\
& =\bigcup_{y \in B}\left(\left[\bigcup_{x \in A}\{x\}\right]+y\right) \\
& =\bigcup_{y \in B} \bigcup_{x \in A}\{x+y\} \\
& =\bigcup_{x \in A}\left(\left[\bigcup_{y \in B}\{y\}\right]+x\right) \\
& =\bigcup_{y \in B}(B+x)
\end{aligned}
$$

The dilation of $A$ by $B$ is defined as

$$
\mathcal{D}(A, B)=A \oplus B
$$


where $B$ is called structuring element. Minkowski subtraction can be written as

$$
\begin{aligned}
A \in B & =\bigcap_{y \in B}(A+y) \\
& =\bigcap_{y \in B}\{x: x \in(A+y)\} \\
& =\bigcap_{y \in B}\{x:-y+x \in A\} \\
& =\{x:-B+x \subset a\}
\end{aligned}
$$

where $-B$ is defined as $-B=\{-b: b \in B\}$. This is equivalent to rotating $B$ by $180^{\circ}$ about the origin, and finding the set of points by which the translation of the rotated $B$ can fit into $A$. The erosion operation of $A$ by $B$ is defined as

$$
\mathcal{E}(A, B)=A \Theta(-B)
$$

where $B$ is also called structuring element. The procedures of dilation and erosion are illustrated in Figure 4.1. Three objects are shown in Figure 4.1a. Erosion and dilation of these objects by a small square structuring element in Figure $4.1 \mathrm{~b}$ are shown in Figure 4.1c and Figure 4.1d, respectively. The dilation and erosion of an object by a structuring element can be seen by sliding the structuring element along the border of the object, and the outer contour drawn by the structuring element defines the dilated object while the inner contour drawn by the structuring element defines the eroded object. In Figure $4.1 \mathrm{c}$, eroded objects are represented by the solid objects and their originals are represented by the outer contours. Symbolically, erosion is like "peeling" the objects at a depth which is half the size of the structuring element, from the outer and inner 


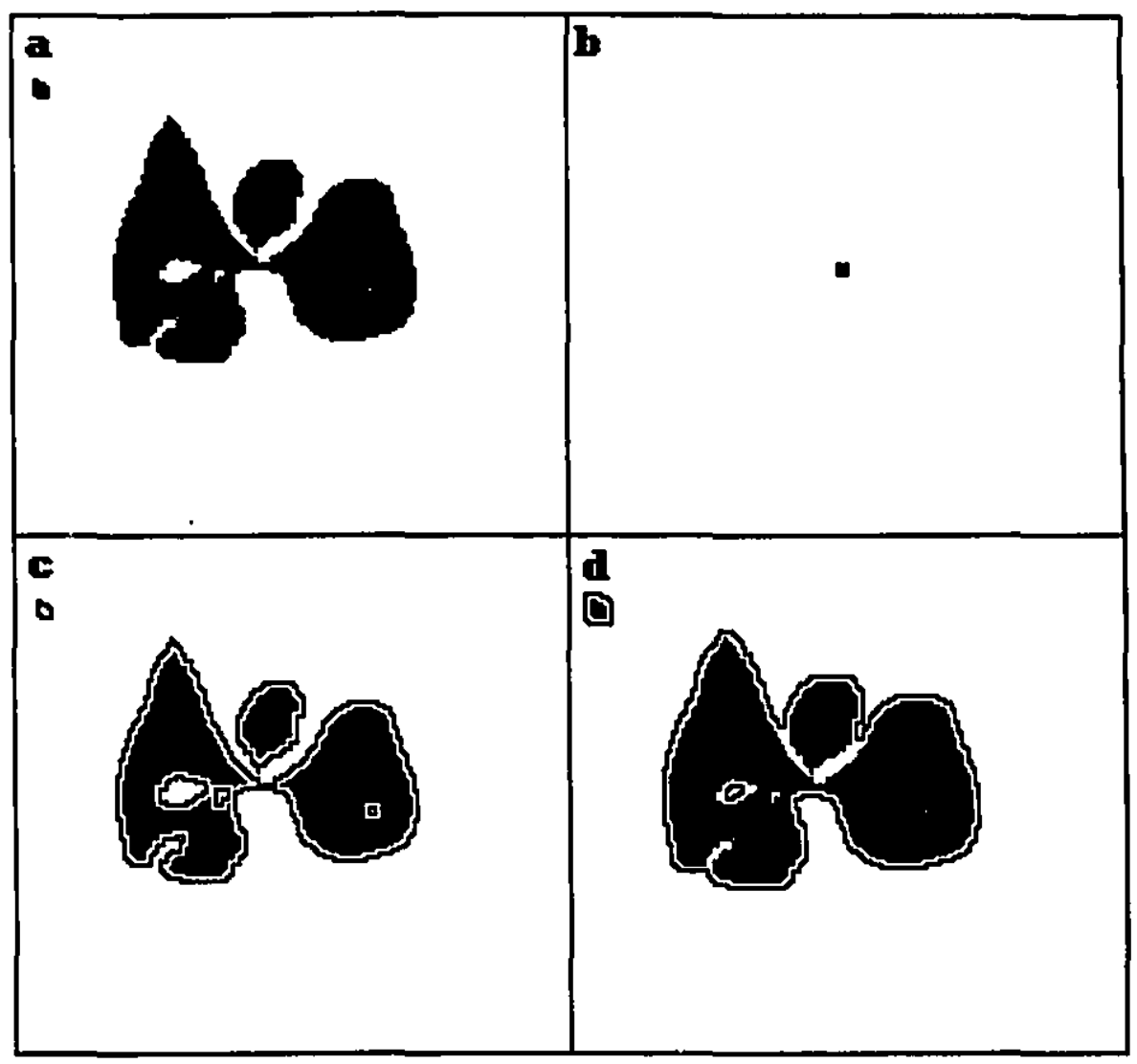

Figure 4.1. (a) original image, (b) structuring element, (c) solid objects are the eroded objects, the outer contour represents the original objects, (d) solid objects are the originals, the outer contour represents the dilated objects. 
"surfaces". After erosion, the small object at the upper left corner disappears, the thin junction on the largest objects is broken, and holes on this object becomes larger. The opposite situation is shown in Figure 4.1d, where solid objects represent the original objects and the outer rontours represent the dilated ones. Dilation is like "pasting" an object with a "coating" whose thickness is half the size of the structuring element, on the outer and inner "surfaces". After dilation, the two objects at the center are united, the two small holes disappear while the largest one becomes smaller, and the crack on the largest object converges.

\section{$\S \S$ 4.3.2 Complex Operations and Algorithms}

Complex operations and algorithms can be built upon the two basic operations, erosion and dilation. Some complex operations and algorithms have become standard processes in morphological image processing. In our context, we will only discuss the two most common complex operations, opening and closing, and a standard algorithm, hole-filling, that we have used in the segmentation of portal images. The opening of $A$ by $B$ is an erosion followed by a dilation,

$$
\mathcal{O}(A, B)=\mathcal{D}[\mathcal{E}(A, B), B]
$$

and the closing of $A$ by $B$ is a dilation followed by an erosion,

$$
\mathcal{C}(A, B)=\mathcal{E}[\mathcal{D}(A, B), B]
$$


Opening and closing are graphically illustrated in Figure 4.2. Original objects in Figure 4.2a are opened and closed with the small square structuring element in Figure 4.2b, and the resultant opened and closed objects are shown in Figure $4.2 \mathrm{c}$ and Figure 4.2d, respectively. It can be seen in Figure 4.2c that opening eliminates the two small objects and breaks the weak junction at the center of the largest object. Sharp tips are also smoothed out. On the other hand, in Figure 4.2d, the crack and the two small holes are filled. Opening behaves similarly as erosion except it maintains the original size of an object. The same type of behavior exists between closing and dilation. Before we proceed to hole-filling, a special type of erosion and dilation must be introduced, i.e. geodesic erosion and geodesic dilation. A structuring element is called a fundamental structuring element if its size is smaller than the shortest distance between any two objects. If the fundamental structuring element is used, an erosion is called a geodesic erosion and a dilation is called a geodesic dilation.

Hole-filling is an operation that fills the holes within objects of an image. Given an image $A$, and $B$ is the boundary of the Euclidean plane, hole-filling of $A$ is an iteration, $C_{i+1}=\bar{A} \cap \mathcal{D}\left(C_{i}, e\right)$ where $C_{0}=B$ and $\bar{A}$ is the complement of $A$. This process will be repeated until convergence, i.e., the next iteration does not make any difference. For example, after the $n$th iteration, $C_{n+1}=C_{n}$. The complement of $C_{n+1}, \overline{C_{n+1}}$, is then taken. The dilation used in hole-filling must also be geodesic. Figure 4.3 shows the 


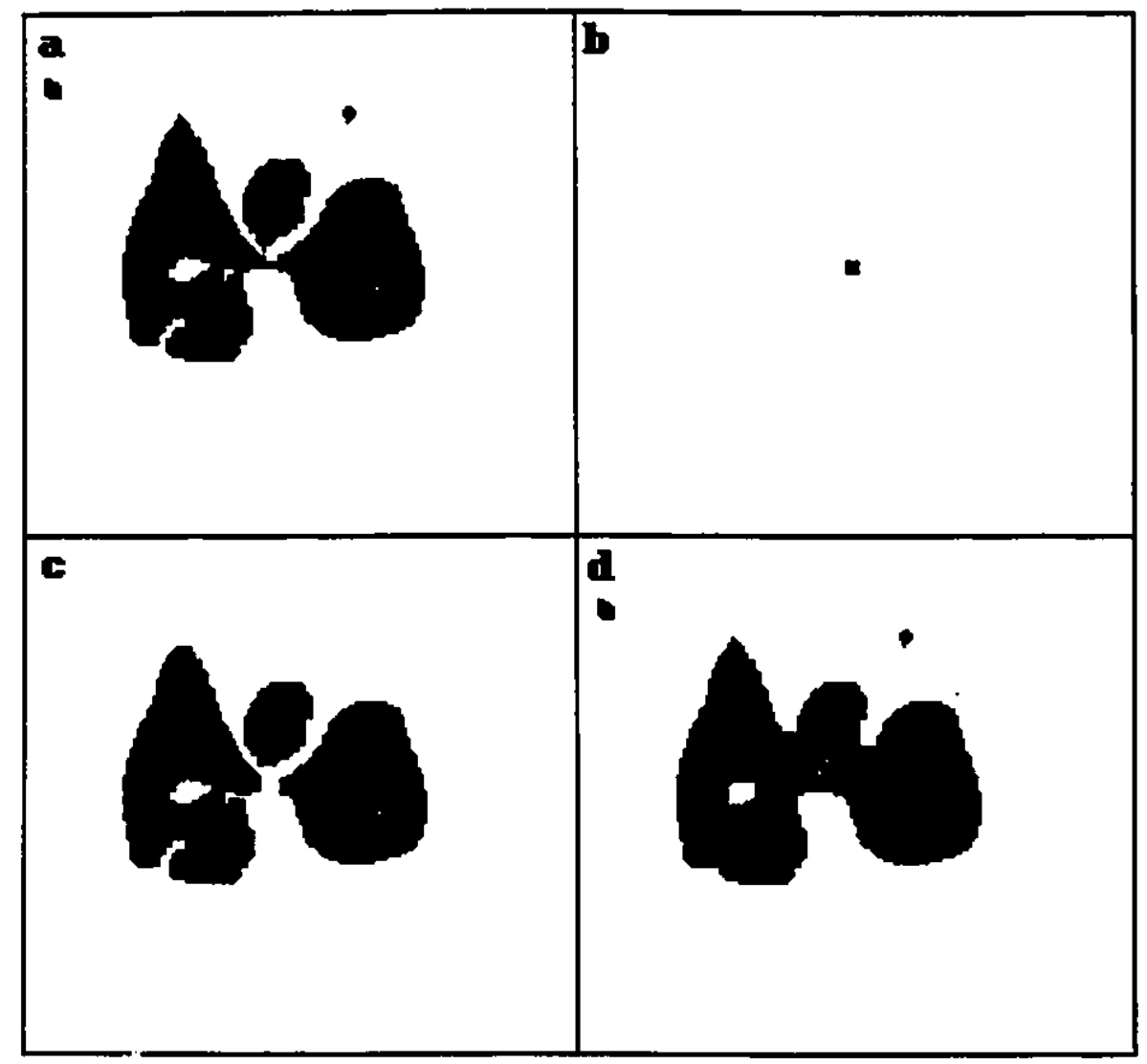

Figure 4.2. (a) original image, (b) structuring element, (c) opening of the original image, (d) closing of the original image. 
procedure of the hole-filling algorithm. To fill the hole on the right object in Figure 4.3a, the image is inverted to its complement Figure 4.3b. The image border in Figure 4.3c is dilated and intersected with Figure 4.3b. The process, an intermediate step of which is shown in Figure 4.3d, continues until the background in Figure 4.3a is flushed as in Figure 4.3e. When Figure $4.3 \mathrm{e}$ is inverted to its complement Figure $4.3 \mathrm{f}$, the hole on the right object has been filled.

\section{$\S \S$ 4.3.3 Digital Morphology}

Mathematical morphology can be easily extended from the Euclidean space to the digital situation with some modification of the definition of the basic operations. When sets in the Euclidean plane are digitized into sets of pixels, two-valued Euclidean morphology becomes binary morphology. In binary morphology, digital binary images are considered sets of pixels. There are three possible values for a pixel, 0,1 and *(undefined). A typical image $f(i, j)$ will be represented as:

$$
f=\left(\begin{array}{llllllll}
* & * & * & 1 & 1 & 1 & 1 & * \\
* & * & 1 & * & 1 & 1 & 1 & * \\
1 & * & 1 & 1 & 1 & 1 & * & * \\
* & * & * & 1 & 1 & 1 & * & 1 \\
* & 1 & * & 1 & * & 1 & * & * \\
* & * & 1 & * & * & 1 & * & 1 \\
* & * & * & * & * & * & 1 & * \\
* & * & * & * & 1 & * & * & *
\end{array}\right)_{3,10}
$$

where $i$ and $j$ are the column number and row number of a pixel, respectively, and $(i, j)$ does not necessarily have to start from $(1,1)$. For example, in Eq. 4.10 the subscript 


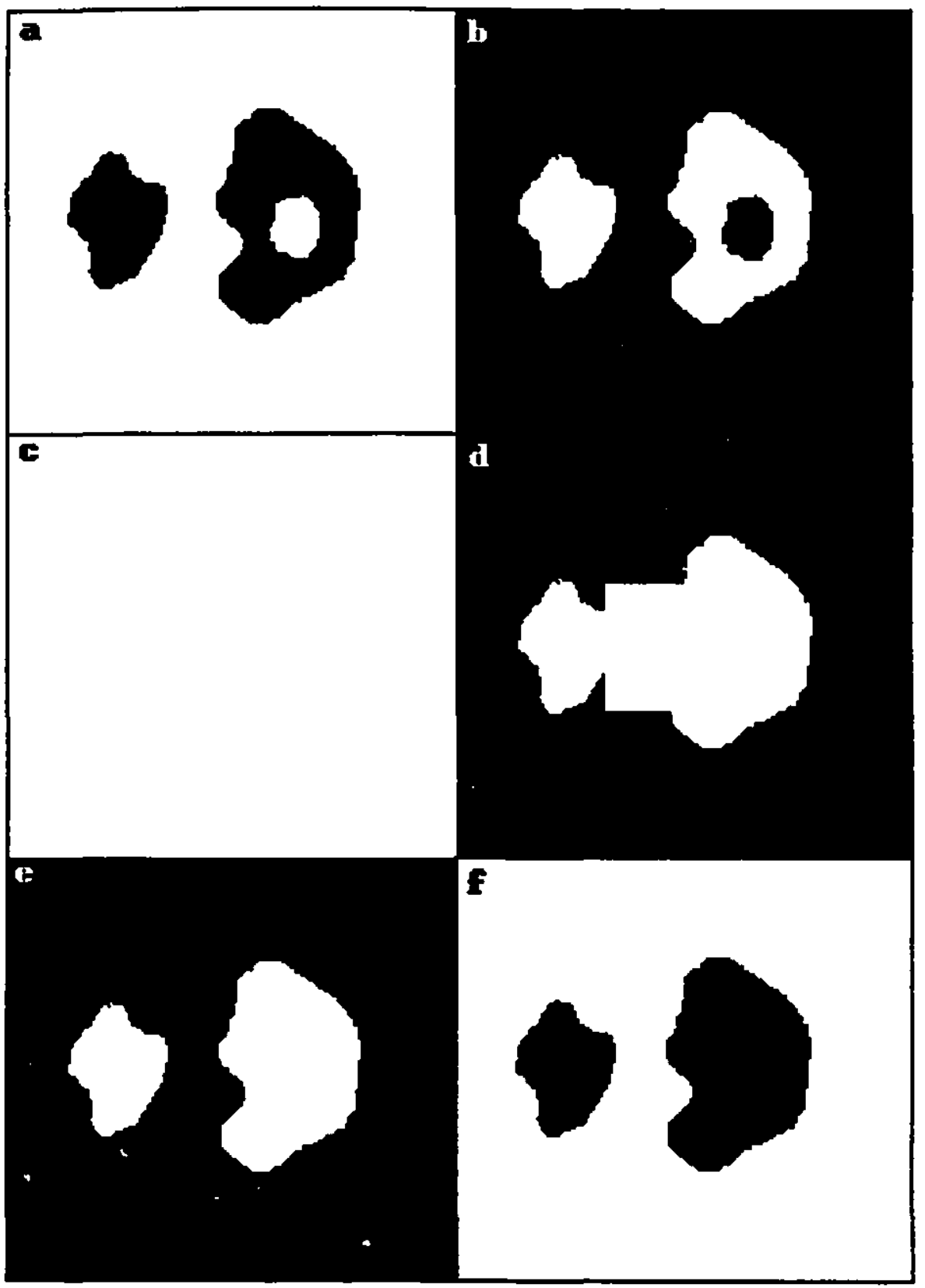

Figure 4.3. Illustration of the hole-filling operation. (a) original image, (b) complement of the original image, (c) image containing a rectangular frame on the image border, (d) intersection of (b) with (c) after some iterations, (e) the convergence of the iterations, (f) complement of (e) representing completion of the hole-filling operation. 
"3, 10" implies that this matrix starts from column 3 and row 10 . Translation of $f$ rightward by $u$ and downwards by $v$ is given by

$$
[\operatorname{TRAN}(f: i, j)](u, v)=f(u-i, v-j)
$$

Rotation of $f$ by $90^{\circ}$ is given by

$$
[\operatorname{NINETY}(f)](i, j)=f(j,-i)
$$

Digital union of a series of images $f_{k}, k=1,2,3 \cdots$ is represented as

$$
\left[\begin{array}{ll}
\bigvee & f_{k}
\end{array}\right](i, j)= \begin{cases}1, & \text { if there exists at least one } k^{\prime} \text { for which } f_{k^{\prime}}(i, j)=1 \\
*, & \text { if } f_{k}(i, j)=* \text { for all } k\end{cases}
$$

while digital intersection is represented as

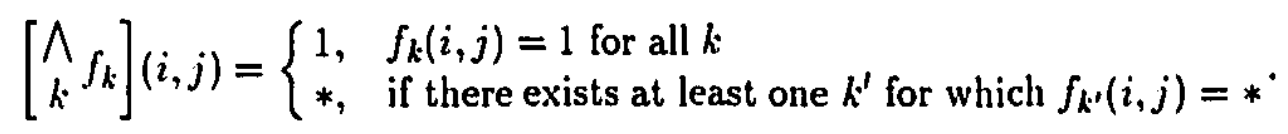

With these primitive operations defined, we can now introduce the digital Minkowski addition,

$$
F \oplus E=\bigvee_{(i, j) \in D_{s}} \operatorname{TRAN}(E ; i, j)
$$

and digital Minkowski subtraction

$$
F \ominus E=\bigwedge_{(i, j) \in \operatorname{DOMAIN}(E)} \operatorname{TRAN}(E ; i, j)
$$

As in the continuous situation, dilation is the Minkowski addition

$$
\begin{aligned}
\operatorname{DILATE}(F, E) & =\bigwedge_{(i, j) \in \operatorname{DOMAIN}(E)} \operatorname{TRAN}(E:-i,-j) \\
& =\bigwedge_{(i, j) \in \operatorname{DOMAIN}[\operatorname{NinETY}(E)]} \operatorname{TRAN}(E: i, j)
\end{aligned}
$$

where DOMAIN $(E)$ means the domain of the structuring element $E$ and $\operatorname{NINETY}^{2}(E)$ means "rotate $E$ by $90^{\circ}$ twice". 


\section{$\S \S$ 4.3.4 Gray Scale Morphology}

The principles of mathematical morphology are not limited to 2-dimensional Euclidean or digital spaces. In fact, mathematical morphology was originally developed in Euclidean $\mathrm{N}$-space. The difference between gray scale morphology and binary morphology is that the primitive operation applied at each pixel is different as the structuring element is translated across the image. In gray scale morphology, the following two operations, extended maximum and minimum, represented as EXTMAX and MIN, respectively, replace the union and intersection operations used in Eq. (4.2) and Eq. (4.3), respectively,

$$
\begin{gathered}
{[E X T M A X(f, g)](i, j)= \begin{cases}\max [f(i, j), g(i, j)], & \text { if both } f \text { and } g \text { are defined at }(i, j) \\
f(i, j), & \text { if } f(i, j) \neq * \text { and } g(i, j)=* \\
g(i, j), & \text { if } g(i, j) \neq * \text { and } f(i, j)=* \\
*, & \text { if } f(i, j)=g(i, j)=*\end{cases} } \\
{[M I N(f, g)](i, j)= \begin{cases}\min [f(i, j), g(i, j)], & \text { if both } f \text { and } g \text { are defined at }(i, j) \\
*, & \text { if either } f \text { or } g \text { is not defined at }(i, j)\end{cases} }
\end{gathered}
$$

Dilation and erosion of an image $f$ by a structuring element $e$ can be expressed pixelwise

$$
\begin{gathered}
D(f, e)_{x, y}=\max _{i, j}[f(x-i, y-j)+e(i, j)] \\
E(f, e)_{x, y}=\min _{i, j}[f(x-i, y-j)-b(-i,-j)]
\end{gathered}
$$

where $(x, y)$ is the index of an arbitrary pixel of $f$ and $(i, j)$ is the index of an arbitrary element of the structuring element kernel. The index of central element of the structuring element kernel is defined as $(0,0)$. 


\section{$\S \S$ 4.3.5 Morphological Gradient}

A very useful gray scale morphological operation is the morphological gradient which can be used as an edge detector. Given an image $f$ and a structuring element $c$, the morphological gradient of $f$ is the subtraction of the erosion of $f$ from the dilation of $f$

$$
\mathcal{G}(f, e)=\mathcal{D}(f, e)-\mathcal{E}(f, e)
$$

The edge response can be adjusted by changing the size and shape of the structuring element. The commonly used structuring element is

$$
\left(\begin{array}{lll}
1 & 1 & 1 \\
1 & 1 & 1 \\
1 & 1 & 1
\end{array}\right)
$$

which is based on the eight-connected neighbors of around a pixel. The morphological gradient is illustrated with the following example. Given an image

$$
f=\left(\begin{array}{lllllll}
0 & 0 & 0 & 0 & 0 & 0 & 0 \\
0 & 8 & 8 & 8 & 8 & 8 & 0 \\
0 & 8 & 8 & 8 & 8 & 8 & 0 \\
0 & 8 & 8 & 8 & 8 & 8 & 0 \\
0 & 8 & 8 & 8 & 8 & 8 & 0 \\
0 & 8 & 8 & 8 & 8 & 8 & 0 \\
0 & 0 & 0 & 0 & 0 & 0 & 0
\end{array}\right)_{1,1}
$$

dilation and erosion are represented as

$$
\mathcal{D}(f, e)=\left(\begin{array}{ccccccc}
9 & 9 & 9 & 9 & 9 & 9 & 9 \\
9 & 9 & 9 & 9 & 9 & 9 & 9 \\
9 & 9 & 9 & 9 & 9 & 9 & 9 \\
9 & 9 & 9 & 9 & 9 & 9 & 9 \\
9 & 9 & 9 & 9 & 9 & 9 & 9 \\
9 & 9 & 9 & 9 & 9 & 9 & 9 \\
9 & 9 & 9 & 9 & 9 & 9 & 9
\end{array}\right)_{1,1}
$$




$$
\mathcal{E}(f, e)=\left(\begin{array}{lllllll}
0 & 0 & 0 & 0 & 0 & 0 & 0 \\
0 & 0 & 0 & 0 & 0 & 0 & 0 \\
0 & 0 & 9 & 9 & 9 & 0 & 0 \\
0 & 0 & 9 & 0 & 9 & 0 & 0 \\
0 & 0 & 9 & 9 & 9 & 0 & 0 \\
0 & 0 & 0 & 0 & 0 & 0 & 0 \\
0 & 0 & 0 & 0 & 0 & 0 & 0
\end{array}\right)_{1,1}
$$

the morphological gradient is given by

$$
\mathcal{G}(f, e)=\left(\begin{array}{lllllll}
9 & 9 & 9 & 9 & 9 & 9 & 9 \\
9 & 9 & 9 & 9 & 9 & 9 & 9 \\
9 & 9 & 0 & 0 & 0 & 9 & 9 \\
9 & 9 & 0 & 0 & \vdots & 9 & 9 \\
9 & 9 & 0 & 0 & 0 & 9 & 9 \\
9 & 9 & 9 & 9 & 9 & 9 & 9 \\
9 & 9 & 9 & 9 & 9 & 9 & 9
\end{array}\right)_{1,1}
$$

What remains in $\mathcal{G}$ is simply the representation of the edge of $f$. A very important property of mathematical morphology is that, when uniform structuring element is used, large size erosion and dilation can be implemented as iterations of erosion and dilation with a small structuring element, respectively. For example, dilation with the $3 \times 3$ kernel

$$
\left(\begin{array}{lll}
1 & 1 & 1 \\
1 & 1 & 1 \\
1 & 1 & 1
\end{array}\right)
$$

applied twice is equivalent to single dilation with the $5 \times 5$ kernel

$$
\left(\begin{array}{lllll}
1 & 1 & 1 & 1 & 1 \\
1 & 1 & 1 & 1 & 1 \\
1 & 1 & 1 & 1 & 1 \\
1 & 1 & 1 & 1 & 1 \\
1 & 1 & 1 & 1 & 1
\end{array}\right)
$$

because the 8 -connected neighbors of the $3 \times 3$ kernel are the elements on the border of the $5 \times 5$ kernel, and the operation applied around the neighborhood of any pixel of an 
image is a comparison. Since the number of calculations is proportional to the square of the size of the structuring element, the iterative method can significantly shorten calculation time. For an $n \times n$ image, in which the border effect is ignored, dilation with the $3 \times 3 \mathrm{kernel}$ requires $(9-1) \cdot n^{2}$ comparisons while dilation with the $5 \times 5 \mathrm{kemel}$ requires $(25-1) \cdot n^{2}$ comparisons. Therefore, the iteration method is approximately $\left[(25-1) \cdot n^{2}\right] /\left[2 \cdot(9-1) \cdot n^{2}\right]$ times faster than the direct method. Since calculation time is a major issue in portal image processing, all the morphological operations we used in this project are performed iteratively by using the $3 \times 3$ constant structuring element. With the basic concepts and operations of mathematical morphology introduced, we can now proceed to the next chapter where we discuss how these operations are employed to build a robust algorithm for the extraction the radiation field from double exposure portal images.

\section{§ 4.4 Edge Detection}

\section{$\S \S$ 4.4.1 Gradient Operators}

An edge in a gray scale image is defined as a discontinuity in gray value. As a discontinuity in a two variable function $f(x, y)$, an edge can be accentuated by its gradients $\frac{\partial f}{\partial x}$ and $\frac{\partial f}{\partial y}$, an edge operator for a gray scale image $I(i, j)$ can be designed as

$$
\Delta_{1}(i, j)=I(i, j+1)-I(i, j)
$$


along the horizontal direction and

$$
\Delta_{2}(i, j)=I(i+1, j)-I(i, j)
$$

along the vertical direction. These edge detectors are called Roberts Gradient Edge Detectors. ${ }^{13}$ Edge detection is implemented by convolution with the following kemels

$$
\left[\begin{array}{ccc}
0 & 0 & 0 \\
0 & -1 & 1 \\
0 & 0 & 0
\end{array}\right],\left[\begin{array}{ccc}
0 & 0 & 0 \\
0 & -1 & 0 \\
0 & 1 & 0
\end{array}\right]
$$

which enhance edges in the horizontal and vertical directions, respectively. The magnitude $G$ and orientation $\Theta$ of the gradient are defined as,

$$
\begin{aligned}
G(i, j) & =\sqrt{\left[\Delta_{1}(i, j)\right]^{2}+\left[\Delta_{2}(i, j)\right]^{2}} \\
\Theta & =\arctan \left(\frac{\Delta_{2}(i, j)}{\Delta_{1}(i, j)}\right)
\end{aligned}
$$

The Roberts edge detectors are sensitive to noise since only the difference with one immediate neighbor is considered. Larger kernels have been designed to overcome the sensitivity to noise, such as the Prewitt ${ }^{14,15}$ and Sobel ${ }^{15}$ edge detectors, shown below for the horizontal and vertical direction, respectively:

$$
\begin{gathered}
\text { Prewitl : }\left(\begin{array}{ccc}
1 & 1 & 1 \\
0 & 0 & 0 \\
-1 & -1 & -1
\end{array}\right),\left(\begin{array}{ccc}
1 & 0 & -1 \\
1 & 0 & -1 \\
1 & 0 & -1
\end{array}\right) \\
\text { Sobel : }\left(\begin{array}{ccc}
1 & 2 & 1 \\
0 & 0 & 0 \\
-1 & -2 & -1
\end{array}\right),\left(\begin{array}{lll}
-1 & 0 & 1 \\
-2 & 0 & 1 \\
-1 & 0 & 1
\end{array}\right)
\end{gathered}
$$


After edge enhancement, the gradient image is usually thresholded to eliminate noise. Some edge detectors, such as the Laplacian operator, can also be based on the second order derivatives. For a two variable function $f(x, y)$ its Laplacian is

$$
\nabla^{2} f(x, y)=\frac{\partial^{2} f(x, y)}{\partial x^{2}}+\frac{\partial^{2} f(x, y)}{\partial y^{2}}
$$

The 4-neighbor Laplacian edge detector is designed as

$$
L(i, j)=I(i-1, j)+I(i+1, j)+I(i, j-1)+I(i, j+1)-4 I(i, j)
$$

and can be implemented as convolution with the Laplacian kernel

$$
\left(\begin{array}{ccc}
0 & 1 & 0 \\
1 & -4 & 1 \\
0 & 1 & 0
\end{array}\right)
$$

Instead of a local maxima, the response of the Laplacian operator to an edge is a pair of peaks, one positive and the other negative. The zero-crossing point corresponds to the position of the edge.

\section{$\S \S$ 4.4.2 Optimal Edge Detectors}

Gradient operators and Laplacian operators are very sensitive to noise because only a very small neighborhood around a pixel is considered. Based on the assumption that local variations corresponding to edge transitions are slower than those corresponding to noise, optimal edge detectors have been designed to suppress noise at the same time as to obtain good edge localization by smoothing the image with some filter before taking 
the gradient ${ }^{16}$. For simplicily, let us consider a one dimension signal $f(x)$ and smooth it with a filter $h(x)$

$$
g(x)=f(x) \odot h(x)=\int_{-\infty}^{\infty} f(x-t) h(t) d t
$$

and the gradient of the smoothed signal is

$$
g^{\prime}(x)=\int_{-\infty}^{\infty} f^{\prime}(x-t) h(t) d t
$$

If the filter has a finite range $[-a, a], g^{\prime}(x)$ can, by integration by parts, be reduced to

$$
g^{\prime}(x)=\int_{-a}^{a} f(x-t) h^{\prime}(t) d t
$$

Thus, edge detection is equivalent to convolving the image with the first derivative of a filter. The optimal edge detector is characterized by the following three criteria: ${ }^{17}$

- The probability of failing to detect real edges and falsely responding to nonedge fluctuation should be small. Since probability of success depends on signal to noise ratio, this criterion corresponds to the maximization of the signal-to-noise ratio.

- The location of the edge points accentuated by the operator should be as close as possible to the center of true edge

- Only one response to a single edge should exist. 
There are different ways to characterize these criteria mathematically, therefore the implementation of the optimal edge detector is not unique. But the performance of different implementation can be evaluated by these criteria. One approximation to the optimal edge detector is the first derivative of the Gaussian (DOG): ${ }^{16}$

$$
\frac{d h(x)}{d x}=-\frac{x}{\sigma^{2}} \operatorname{cxp}\left(-\frac{x^{2}}{2 \sigma^{2}}\right)
$$

where $\sigma$ is the standard deviation of the Gaussian function. A unique feature of portal images is that the field edge has bigger penumbra than anatomy edges since the collimators are closer to the focal spot than is the patient, resulting in a wide slope in the gray value across the field border. This feature can be used to differentiate field edge from anatomy edges. The DOG operator has been used by Leszczynski et al to segment portal images acquired from an on-line imager. ${ }^{2}$ While the DOG operator can accentuate broad edges and depress sharp ones, it requires convolution with a large kernel. For a $256 \times 256$ portal image, the standard deviation $\sigma$ of the Gaussian function has been shown to be $2 \sim 3$ which corresponds to a kernel size of approximately 11 . To accelerate the process of edge detection, we investigated the performance of two types of edge detectors on double exposure portal images, the Canny-Deriche and the morphological gradient. The CannyDeriche operator is a better implementation of the optimal edge detector. It gives better performance than the DOG operator according to the three criteria of the optimal edge 
detector, ${ }^{18}$ and was implemented in a highly recursive fashion. This filter is described by

$$
h(x)=(1+\alpha|x|) \exp (-\alpha|x|)
$$

and the detector is given by

$$
\frac{d h(x)}{d x}=-\alpha^{2} x \cdot \exp (-\alpha|x|)
$$

Optimization between noise suppressing and good edge localization can be achieved by adjusting the spreading coefficient $\alpha$. The smaller the spreading coefficient is, the stronger the smoothing effect is. For double exposure portal images, we found that the best $\alpha$ is around 0.5 . Since the Canny-Deriche edge detector is implemented recursively, the calculation time is independent of the value of $\alpha$.

\section{$\S \S$ 4.4.3 Morphological Edge Detectors}

The simplest morphological edge detectors are the dilation residue and erosion residue operators. ${ }^{19}$ The dilation residue operation is the subtraction of an image from its dilation with a structuring element, while the erosion residue is the subtraction of the erosion of the image from the original image. The difference image is the edge image. The dilation of a grayscale image $f(i, j)$ with a grayscale structuring element $e(i, j)$ is

$$
D[f, e](i, j)=M A X[f(i-l, j-m)+e(l, m)]
$$

while erosion is

$$
E[f, e](i, j)=\min [f(i-l, j-m)+e(l, m)] E .
$$


Erosion residue operation is given by

$$
G_{c}[f, e](i, j)=f(i, j)-\min [f(i+l, j+m)-e(l, m)]
$$

while dilation residue operation is given by

$$
G_{d}[f, e](i, j)=f(i, j)-\max [f(i-l, j-m)+e(l, m)]
$$

For good edge localization, small structuring elements should be used. This makes erosion residue and dilation residue operations sensitive to noise. Larger structuring element can be used to suppress noise, but the edge obtained will be shifted inward or outward with erosion residue or dilation residue operations, respectively. Good edge localization can be achieved with the morphological gradient operation ${ }^{12}$ (Mgradient) which is the subtraction of the erosion of the image from the dilation of the image

$$
G[f, e](i, j)=\max [f(i-l, j-m)+e(l, m)]-\min [f(i+p, j+q)+e(p, q)] .
$$

Mgradient will place the center of the edge at the exact boundary of a structure. If constant structuring element is used, i.e. $e(i, j)=$ constant, such as the 8-connected structuring element

$$
\left(\begin{array}{lll}
1 & 1 & 1 \\
1 & 1 & 1 \\
1 & 1 & 1
\end{array}\right)
$$

Mgradient can be simplified to

$$
G[f, e](i, j)=\max [f(i-l, j-m)]-\min \left[\int(i+p, j+q)\right]
$$


This is similar to the subtraction of two convolution operations except that maximum and minimum take the place of summation. Since any structures smaller than the size of the structuring element will be eliminated in the dilation and erosion processes, the Mgradient also has smoothing capability. A comparison of the performance of the Mgradient with that of the Sobel and Canny-Deriche is shown in Figure 4.4. The edge images have been thresholded with a threshold value at which the closed contour of the treatment field is just found. The edge images have been thresholded with a threshold value at which the closed contour of the treatment field is just found. It can been seen that the Canny-Deriche and the Mgradient are much less sensitive to sharp edges and noise. In the studies, we have preferred the Mgradient detector because of its speed and its low sensitivity to noise. Once the edge has been determined using the Mgradient, we proceed to extract the radiation field to finally automatically segment it from the outer field image.

\section{§ 4.5 Algorithm Development}

\section{$\S \S$ 4.5.1 Basic Approach}

There are two approaches in image segmentation, region oriented and edge oriented. The region oriented approach classifies pixels into different categories according to some properties of the pixels and sorts them into different regions. In the edge oriented approach, different regions are differentiated by their boundaries. The region oriented approach is not appropriated for the segmentation of double exposure portal images 


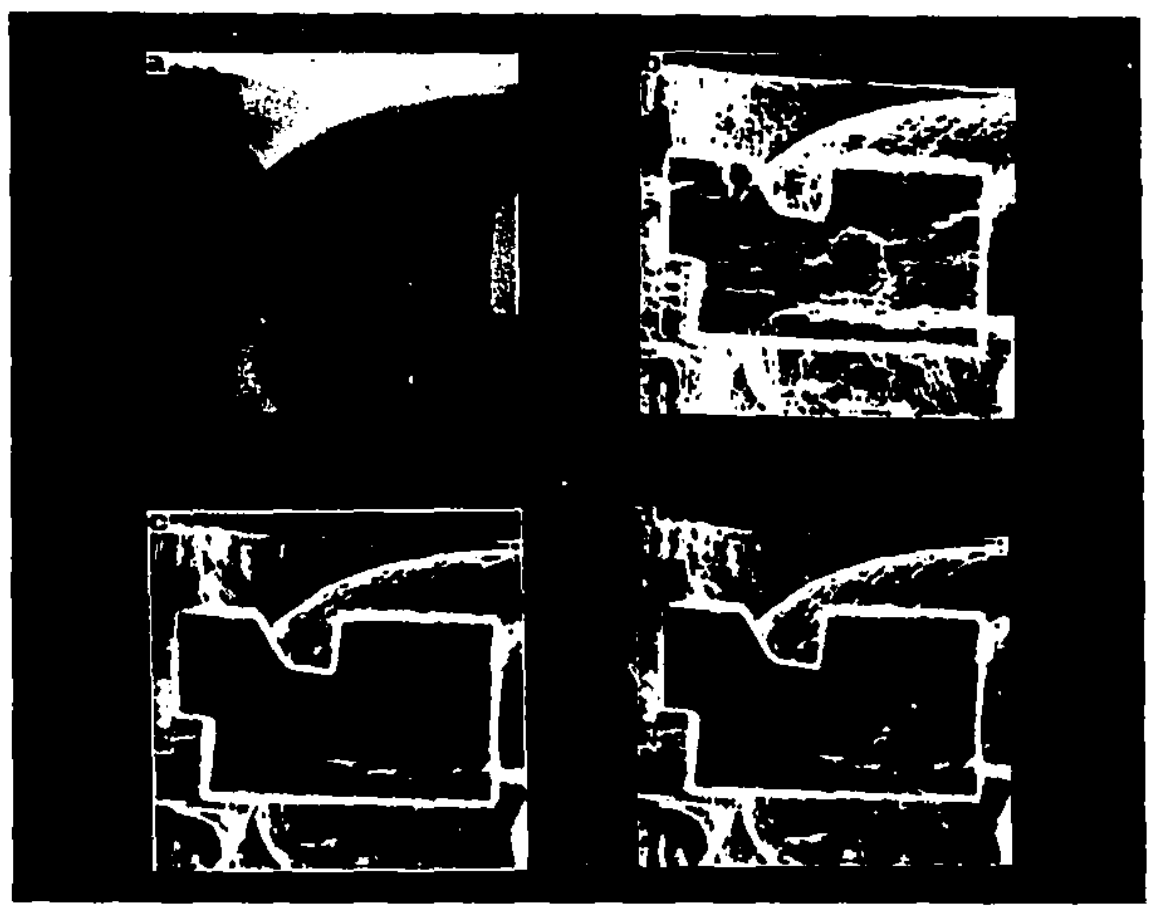

Figure 4.4. Performance of three different edge detectors on a double exposure portal image. (a) The original portal image; (b) Sobel, threshold=1; (c) Canny-Deriche, threshold=4; (d) Mgradient, threshold=6. 
because it is difficult to find any measure except gray level that is very different inside the treatment field from that which is outside the treatment field. Moreover, even the gray value is not uniform inside the treatment field since the treatment field is only a shadow on the anatomy. An additional difficulty is that the position of the treatment field relative to the anatomy is specified by the field boundary requiring that the field boundary be accurately localized. Because of these reasons, most radiation field extraction algorithms that have been reported calculate the gradient of pixel value variation and then track local maximum gradient point to form a contour. However, the radiation field edge is highly contaminated with anatomical edges. Strong anatomical variations on the radiation field border make the field edge not well defined and can mislead the tracing of local maximum gradient points. ${ }^{20}$ This is clearly demonstrated in Figure 4.5.

We have taken an alternative approach to develop a robust algorithm. After edge enhancement with Mgradient, a thresholding operation is applied to remove noise and unwanted edges. Thresholding is a transform that assigns a single value (usually "l" is used) to all the pixels whose gray value is greater than the threshold and assigns "0" to all the others. Given a grayscale image $I(i, j)$, thresholding by a threshold value $T$ results in a binary image $O(i, j)$,

$$
O(i, j)= \begin{cases}1, & \text { if } I(i, j)>T \\ 0, & \text { if } I(i, j)<T\end{cases}
$$



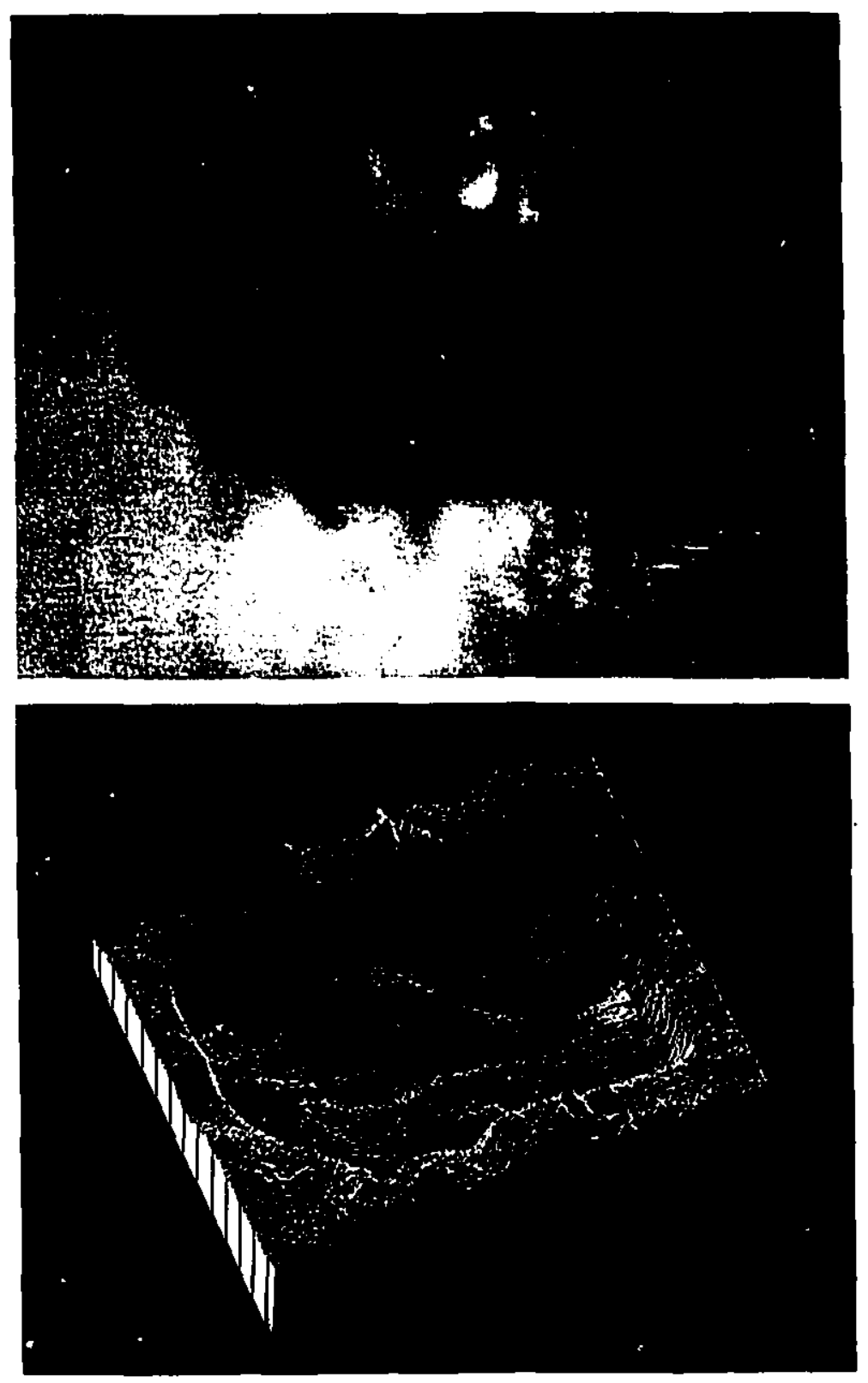

Figure 4.5. Contamination of gradient by anatomical variations. 
In the case of a double exposure portal image, e.g. Figure 4.6a, the feature that we want to extract is the closed contour of the treatment field. But the edge intensity along the treatment field contour is not uniform since the treatment field is a transparent shadow on the background anatomy. Therefore, the threshold value is selected as the one at which a closed field contour is formed. Usually, portal images have a low inherent contrast. If a double exposure portal film is appropriately acquired, i.e. the two exposures are in a reasonable proportion, the treatment field has greater contrast than the anatomy. Therefore, after edge enhancement, the field edge is stronger than the anatomy edges. The closed field contour can be extracted at a threshold value higher than the intensity of the anatomy edges, resulting in a very clean edge. But on some occasions, the field border may fall on some dark structure, therefore causing some parts of the field edge to be significantly weakened and to become comparable with strong anatomy edges. In order to obtain the closed field contour, the threshold value must be reduced. At the same time, noise and anatomy edges will appear within the binary edge image and may be connected to the field edge, as in Figure 4.6b. However, if the binary edge image in Figure 4.6b is inverted, closed edges become gaps separating different objects, and open edges become cracks or holes on objects. The task is changed from extracting the field contour to extracting the object representing the treatment field. And the problem is changed from removing unwanted anatomy edges connected to the field contour to picking up the object corresponding to the treatment field, and closing cracks and filling holes on 


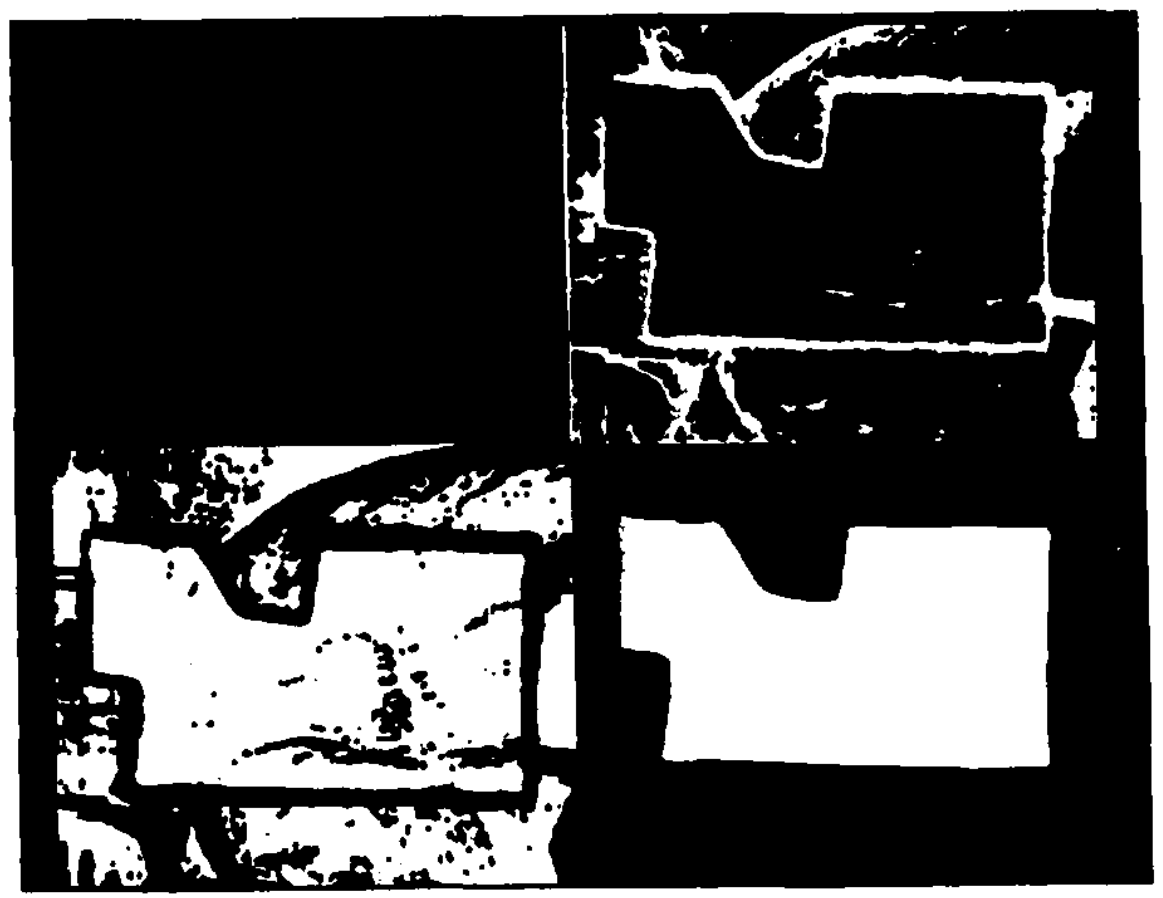

Figure 4.6. Illustration of the basic approach in the segmentation algorithm. Top left: original portal image; top right: binary edge image after proper thresholding of the gradient of the original image; bottom left: complement of the binary edge image; bottom right: the object corresponding to the radiation field. 
the object. This process is made much simpler by using morphological techniques than it is by incorporating some form of high-level knowledge into feature extraction algorithms.

\section{$\S \S$ 4.5.2 Feature Extraction}

After the binary edgs image is inverted, it is labeled for analysis in order to isolate the field object. The labeling operation is to differentiate different objects by assigning different gray levels to different objects in a binary image as illustrated in Figure 4.7. Objects were then separated by thresholding at each gray level, and the area and average pixel value were calculated. From all the objects that satisfy the area criterion, the one corresponding to the relatively darkest region in the original image was selected as the field object.

\section{$\S \S$ 4.5.3 Edge Localization}

Although the field object has the same shape with the dark treatment field on the original portal image, its size is smaller because the field edge usually has a width of several pixels. It also has some cracks and holes on it due to the anatomy edges and noise. From our own investigation, we found that applying the closing operation three times is sufficient to close any cracks on any field objects. A hole-filling operation is applied to fill any possible holes on the object.

To determine the correct number of dilations for accurate edge localization, we refined the field object immediately after the extraction (Figure 4.6c) by performing a "closing" 


$$
\left[\begin{array}{lllllllllllll}
1 & 1 & 0 & 0 & 0 & 0 & 0 & 0 & 0 & 1 & 1 & 1 & 0 \\
1 & 1 & 0 & 1 & 1 & 0 & 0 & 0 & 0 & 1 & 1 & 1 & 0 \\
1 & 0 & 0 & 1 & 1 & 1 & 0 & 0 & 0 & 0 & 0 & 0 & 0 \\
1 & 0 & 1 & 1 & 1 & 1 & 0 & 0 & 0 & 0 & 0 & 0 & 0 \\
0 & 0 & 0 & 0 & 1 & 0 & 0 & 0 & 0 & 0 & 1 & 1 & 1 \\
0 & 0 & 0 & 0 & 0 & 0 & 0 & 0 & 0 & 0 & 0 & 1 & 1 \\
0 & 0 & 1 & 0 & 0 & 0 & 0 & 0 & 1 & 1 & 0 & 0 & 1 \\
0 & 0 & 1 & 1 & 1 & 1 & 1 & 1 & 1 & 1 & 0 & 0 & 1 \\
0 & 0 & 1 & 1 & 1 & 1 & 1 & 1 & 1 & 0 & 0 & 0 & 1
\end{array}\right]
$$

$$
\left[\begin{array}{lllllllllllll}
1 & 1 & 0 & 0 & 0 & 0 & 0 & 0 & 0 & 2 & 2 & 2 & 0 \\
1 & 1 & 0 & 3 & 3 & 0 & 0 & 0 & 0 & 2 & 2 & 2 & 0 \\
1 & 0 & 0 & 3 & 3 & 3 & 0 & 0 & 0 & 0 & 0 & 0 & 0 \\
1 & 0 & 3 & 3 & 3 & 3 & 0 & 0 & 0 & 0 & 0 & 0 & 0 \\
0 & 0 & 0 & 0 & 3 & 0 & 0 & 0 & 0 & 0 & 4 & 4 & 4 \\
0 & 0 & 0 & 0 & 0 & 0 & 0 & 0 & 0 & 0 & 0 & 4 & 4 \\
0 & 0 & 5 & 0 & 0 & 0 & 0 & 0 & 5 & 5 & 0 & 0 & 4 \\
0 & 0 & 5 & 5 & 5 & 5 & 5 & 5 & 5 & 5 & 0 & 0 & 4 \\
0 & 0 & 5 & 5 & 5 & 5 & 5 & 5 & 5 & 0 & 0 & 0 & 4
\end{array}\right]
$$

Figure 4.7. Illustration of the "label" operation on a binary image. Top: binary image; bottom: labeled image. 
operations ( 3 iterations for $256 \times 256$ images, 5 iterations for $512 \times 512$ images) followed by "hole-filling" operation. The refined field object (Figure 4.8a) was dilated step by step to obtain ten enlarged objects, one of which is shown in Figure 4.8b. The refined field object was eroded step by step to obtain ten successively smaller objects, one of which is shown in Figure 4.8c. Each of the dilation and the erosion operation enlarges and reduces the size of the object by one pixel, respectively, without changing its shape. A series of 21 successively larger objects is thus generated. Subtracting each object from its immediately smaller member results in a contour of one pixel thickness. As shown in Figure 4.8d, these contours were overlaid on top of the original portal image and the values of the pixels along each contour are averaged. These average pixel values were then plotted to generate an average slope profile, an example of which is given in Figure 4.9.

A high degree of similarity in shape was observed among average slope profiles of 29 portal images chosen at random in our patient archives. We used a hyperbolic tangent function of the form

$$
y=a \tanh \left(\frac{x-b}{c}\right)+d
$$

to fit these profile plots, where $a$ corresponds to the height of the step, $b$ corresponds to the central point of the slope, $c$ specifies the steepness of the slope, and $d$ refers to the average bias of the step. It should be pointed out that the central point of the slope 


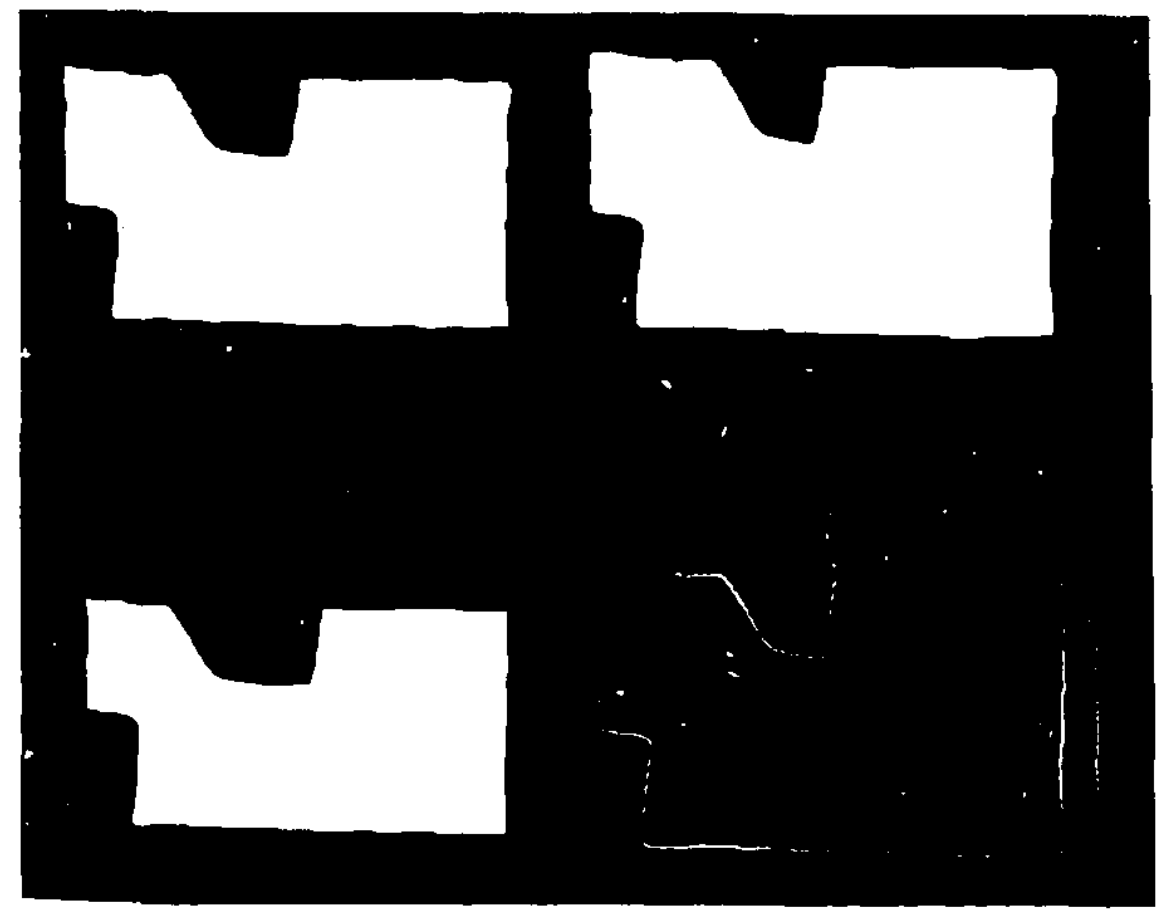

Figure 4.8. Illustration of the procedure of field border placement. Top left: the field mask before enlargement; top right: the field mask after five dilations, bottom left: the field mask after five erosions; bottom right: contours of the original, enlarged and reduced field masks cveriaid on the original image. 


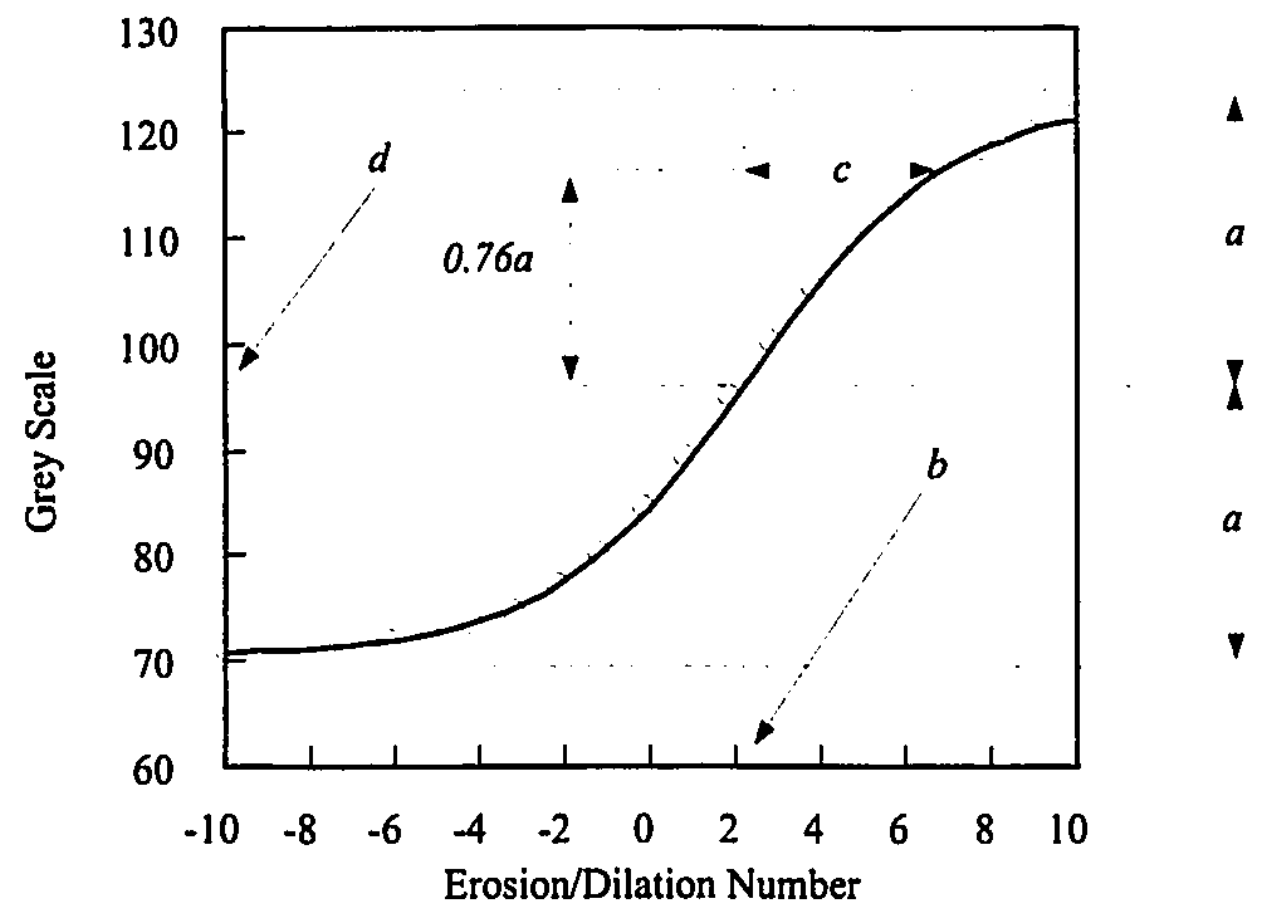

Figure 4.9. Typical plot of average grey scale values versus the number of dilations/erosions of the data and fitting which resulted from Eq. (4.56) shown as solid line. Also depicted are the various fitting parameters. On the horizontal axis, the negative and positive numbers correspond to the number of erosions and dilations, respectively. 
corresponds to the real border of the radiation field. A chi-square fitting algorithm, the Levenberg-Mardquart method, ${ }^{21}$ was used for the curve fitting.

The goodness of fit is represented by the chi-squared value which in turn is related to correlation coefficient. The correlation coefficients obtained from the fit of Eq. (4.56) to data are very close to 1 . In Figure $4.10 \mathrm{a}$ we show a histogram of the correlation coefficients obtained from 29 portal images. The parameter $b$ which, as discussed, corresponds to the central point of the slope, also identifies the number of ditations needed to accurately determine the edge pcsition. A histogram of $b$ among the 29 portal images used is shown in Figure 4.10b. The parameter $b$ obtained from the fitting is a floating point number, which must be rounded to an integer number to refer to the stepwise operation of the "dilation" and "erosion" processes on a digital image. The size of any rounding error is dependent on the size of a pixel, and in our case is less than the size of a pixel, where a pixel is approximately $0.5 \mathrm{~mm}$. We can now determine the optimum number of dilations required for individual images. Ten "erosions" and ten "dilations" and the final fitting requires about 5 seconds on an Indigo Workstation (Silicon Graphics, Inc., Mountain View, California).

The scheme of the algorithm is illustrated in Figure 4.11. To be automatic, the algorithm must be capable of adjusting itself to search for an optimal threshold value with which the field extraction procedure mentioned above can proceed. The optimal 

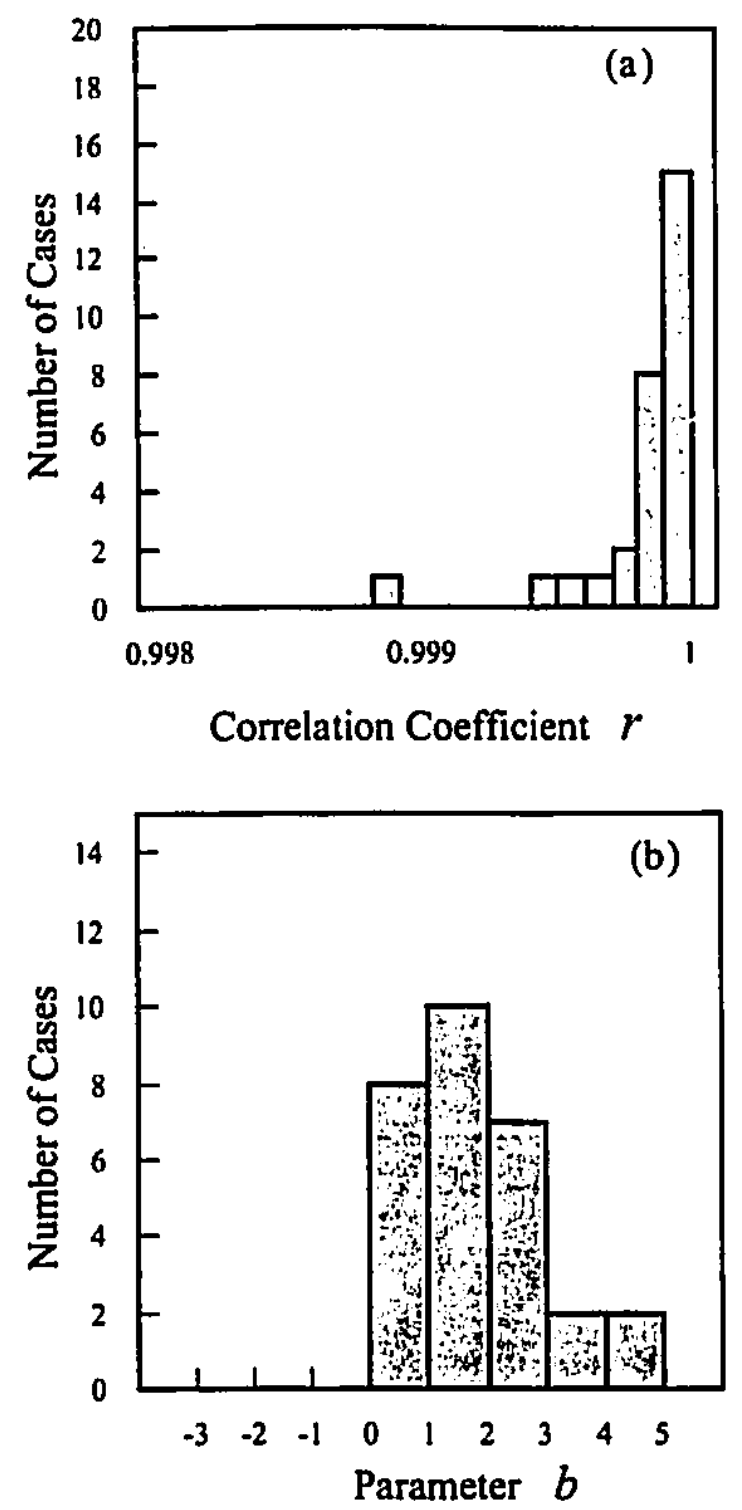

Figure 4.10. (a) Histogram of the correlation coefficient obtained from the curve fitting among 29 portal images; (b) Parameter $b$ obtained from the curve fitting among 29 portal images. 


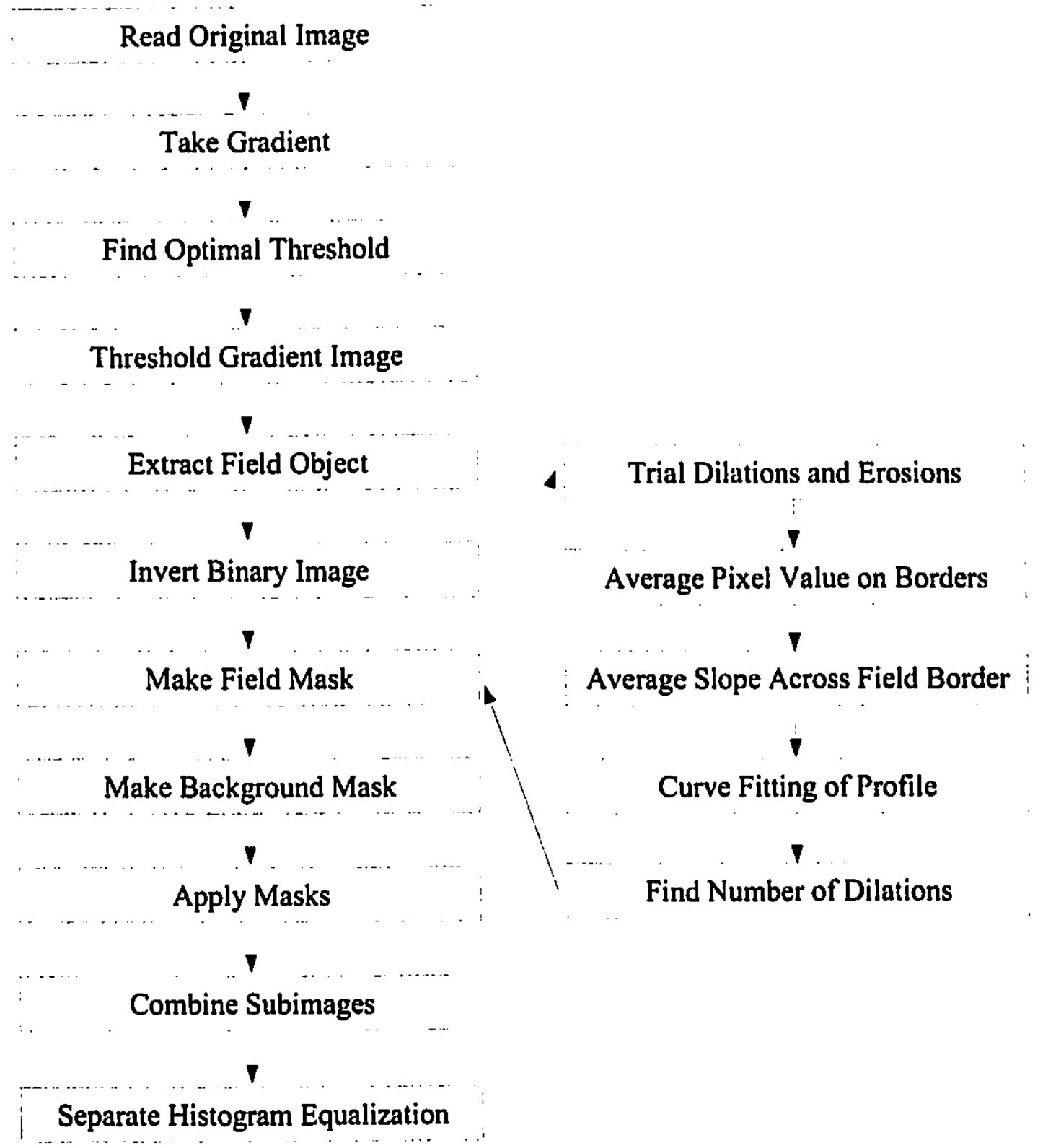

Figure 4.11. Flowchart of the mainstream of the segmentation and contrast enhancement procedure. 
threshold value could be defined as the highest value at which a closed field contour is just found in the binary image. But, if the field contacts the image border, its contour will be an open curve with both ends on the image border. A better definition has been used, i.e. the optimal threshold value is the highest value at which, in the complement of the binary image, the object corresponding to the treatment field is just separated out from the background. Portal images are acquired for different anatomical sites from different therapy machines and thus have different optimal threshold value.

\section{$\S \S$ 4.5.4 Automation of Segmentation}

The searching procedure starts with a high threshold value at which nothing is separated and decrements the threshold value one by one. A predefined area criterion is verified at tach threshold value to determined whetner to stop the search or not. Due to the complexity of the content of portal images, the procedure has been divided into two stages, each with its own criterion. The first approximation, the flowchart of which is shown in Figure 4.12, is based on a very simple model. Usually, portal images have very low contrast, and the intensity of the field edge is much greater than that of anatomy edges. While the threshold is being decreased, the field edge will appear first in the binary edge image. This means that in the complement of the binary edge image, a big object of the size of the whole image will break up into two big pieces corresponding to the radiation field and the surrounding area. Therefore, our first approximation is to 


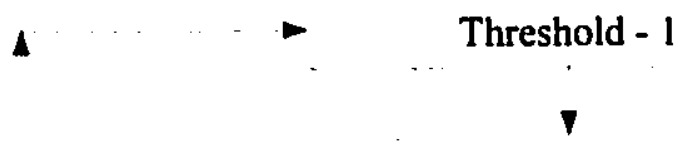

Thresholld Gradient Image

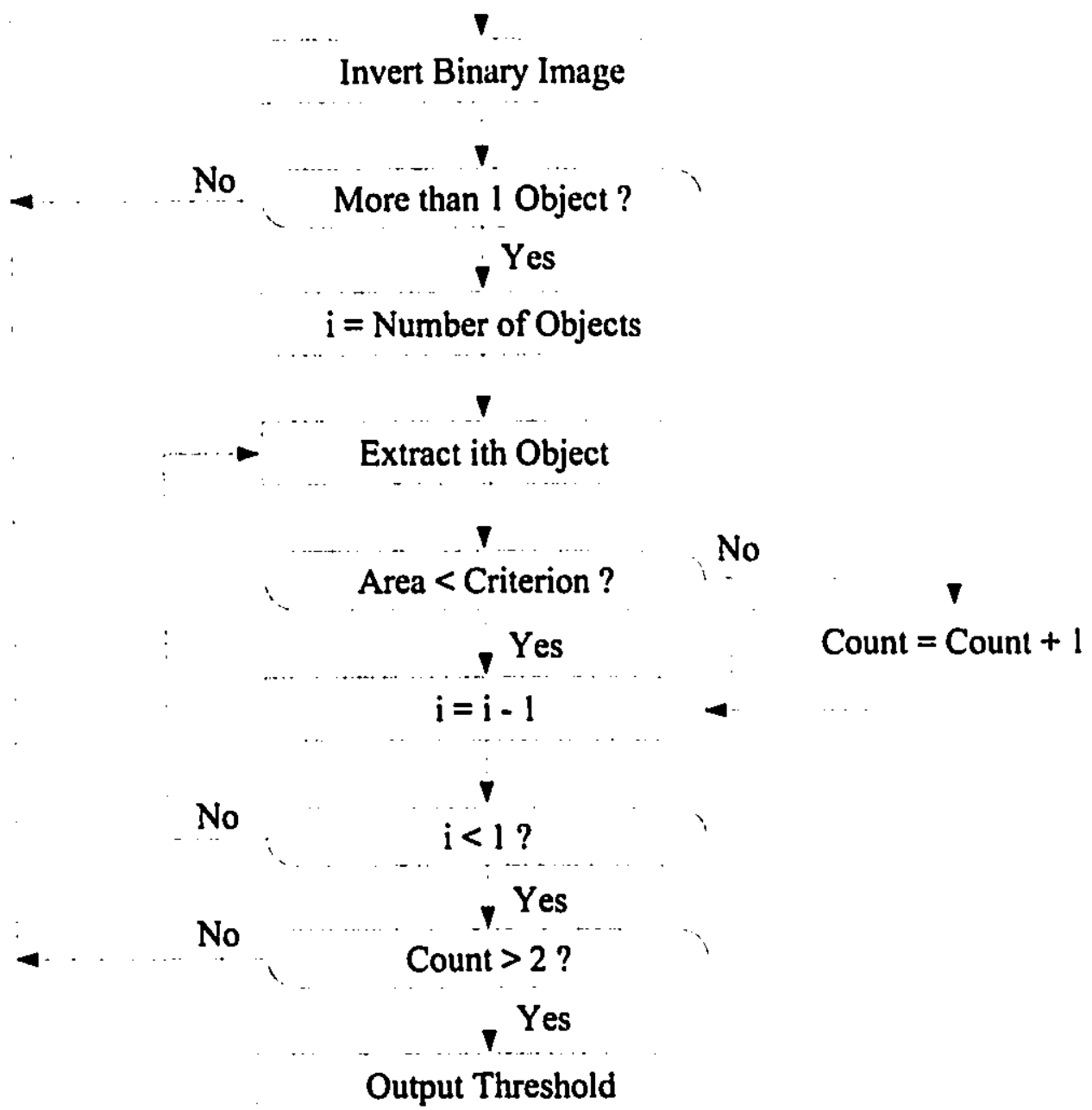

Figure 4.12. Flowchart of the first step of the segmentation procedure. 
determine the threshold value at which this just occurs. Starting from the initial threshold value, the program counts the number of separate objects in the complementary of the binary image whose area is larger than a specific criterion. The criterion we chose is $1 / 14$ of the whole image area. If the number of large objects is smaller than 2 , then the threshold value is decremented, and the number of large objects is recounted, and this process continues until two large objects are found.

In some cases, part of the field border may fall on a dark structure as in Figure 4.13a. This part of the field edge will be significantly weakened so that its intensity is probably comparable to that of the strong anatomy edges outside the field. In the complement of the binary image, the background may fall apart into several pieces before the field object comes out completely. Therefore, the area criterion may be met at a threshold value higher than the optimum and, step one is stopped earlier as in Figure 4.13c.

The second step of the segmentation, whose flowchart is shown in Figure 4.14, is designed to accommodate this difficult situation. After the first approximation, if the field object is still connected to the background, the connection must be weak. The second approximation is to pick up the field object and verify whether it is compact or not. As previously described in Section 4.3.2, the opening operation can break up weak junction which is smaller than the structuring element. Therefore, after the first criterion is met, the program extracts the central object and, opens it four times to verify 


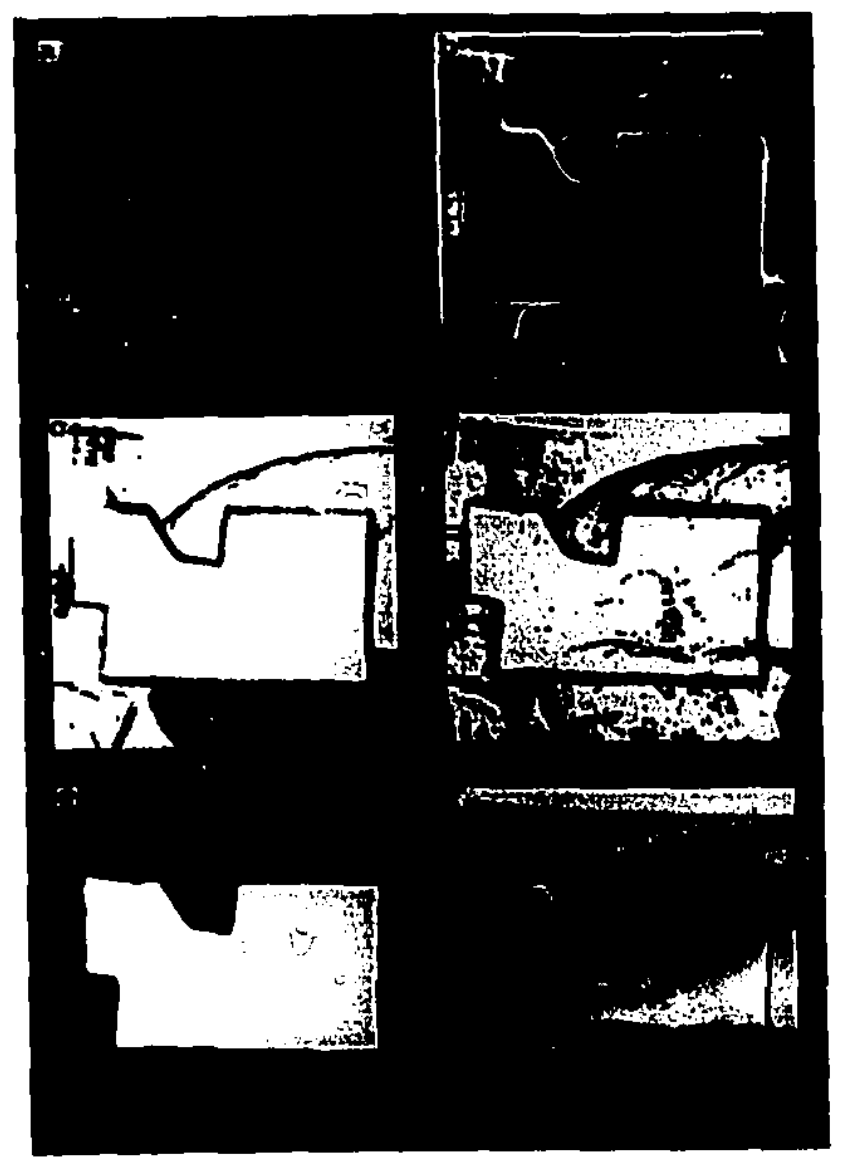

Figure 4.13. (a) original image, (b) binary edge image at the threshold value reached by the first approximation, (c) labeled complement of (b), (d) object image at the optimal threshold value, (e) treatment field mask made from the central object in (d), (f) portal image enhanced with SHE 


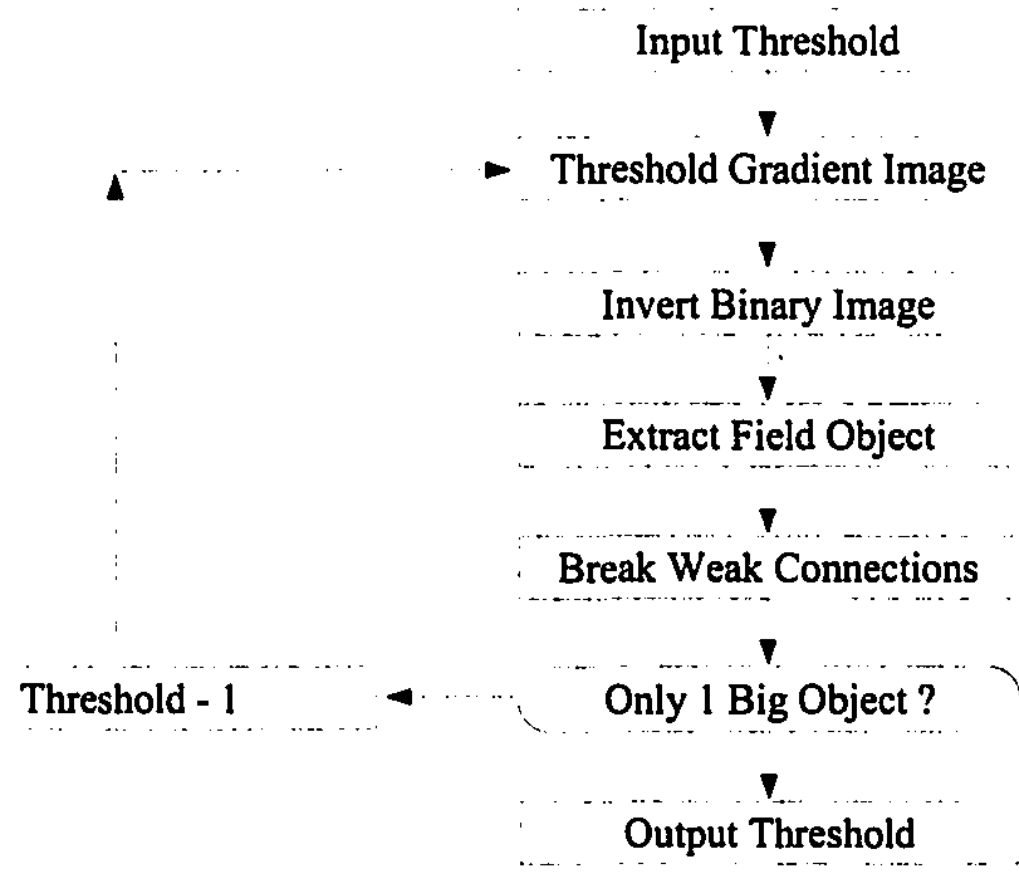

Figure 4.14. Flowchart of the second step of the segmentation procedure. 
whether any piece of a significant size can be broken up from this object. At this stage, if any piece can be broken out from the central object, its size must be relatively small. The criterion is set as $1 / 65$ of the whole image area. If, after the opening operation, only one object larger than this criterion is found, then we assume that we have reached the optimum threshold value. Otherwise, the threshold value will be decreased further, and the test will be repeated until the optimal threshold value is reached. Opening with the 8-connected structuring element for 4 times is equivalent to opening with a $9 \times 9$ structurin $\tilde{\mathcal{E}}$ element. This operation is sufficient to break any inherent junctions on the object because the shape of a prescribed treatment field is sufficiently compact to sustain this operation. Step two does not contradict with step one because it will be satisfied automatically if the optimal threshold value has already been reached by step one alone. This automatic segmentation technique was tested on a large number of portal images acquired from a cobalt unit, $4 \mathrm{MV}, 6 \mathrm{MV}$ and $10 \mathrm{MV}$ linacs. The images cover a variety of treatment sites. The algorithm turns out to be accurate, robust and fast. The computation time varies from one image to another because of the dynamic reasoning procedure, but it is less than 15 seconds on an SGI Indigo workstation.. Besides selective histogram equalization, another purpose of automatic segmentation of double images is to accelerate the process of treatment verification. The automatically extracted treatment field mask will be used as the landmark for portal-simulator image correlation which will 
be discussed in the next chapter.

\section{§4.6 Discussions}

However, one issue needs to be further studied. The radiation field penumbra may be different on different parts of tire field edge, e.g., the penumbra width of an edge shaped by a block is different from that shaped by the collimator jaws. Ideally, the rough field contour should be segmented and the segments can be replaced with perfect mathematical lines or curves based on a priori knowledge. These perfect segments should be localized separately by using the proposed model. We are currently incorporating these ideas into our algorithm and comparing the results of our global approach with those based on local gradients on single- and double-exposure portals.

Our technique of extracting the radiation field is quite different from those used on single-exposure portals. ${ }^{1,2}$ In single-exposure portals the field borders are relatively better defined by the collimators and blocks because there is no anatomical information beyond the radiation field. In double-exposure portals, the radiatinn field is not an inherent feature but an artificial shadow on top of the anatomy. The interference on the field edge from the background anatomy, both inside and outsile the radiation field, is more severe than in single-exposure portals. Furthermore, this interference is not the same along the radiation field border. Even for a field edge shaped with a straight block, it is unliksiy that the pixels with local maximum gradient strength fall on a straight line. Tracking 
the local maximum gradient points does not necessarily lead to the true representation. The tracked contour usually requires smoothing which makes use of a priori knowledge of the edge, e.g., least square fitting of segments into straight lines. ${ }^{2}$ We have taken an alternative approach which smooths the rough edge contour first and then places the smoothed contour at an optimal location by modeling the general behavior of the radiation field penumbra. We have found this approach to be successful on double-exposure and single-exposure portals for all field shapes. The approach can easily be used on electronic portal imaging which can be considered a single-exposure portal terhnique because the images are analyzed individually even in the double-exposure mode. 


\section{References}

I J. Bijhold, K. G. A. Gilhuijs, M. van Herk and Meertens, "Radiation field edge detection in portal images," Physics in Medicine and Biology 36, 1705-1710 (1991).

${ }^{2}$ K. W. Leszczynski, S. Shalev, and N. S. Cosby, "The enhancement of radiotherapy verification images by an automated edge detection technique," Medical Physics 19 $611-621(1992$.

3 L. E. Reinstein, H. I. Amols, P. J. Biggs, R. T. Droege, A. B. Filimonov, W. R. Lutz, and S. Shalev, AAPM Report No. 24 Radiotherapy Portal Imaging Quality(American Institute of Physics, New York, 1988).

4 I. Crooks and B. G. Fallone, "PC-based selective histogram equalization for contrast enhancement of portal films," Medical Physics (abstract) 18, 618 (1991).

5 I. Crooks and B. G. Fallone, "Contrast enhancement of portal images by selertive histogram equalization," Medical Physics20, 199-204(1993).

6 I. Crooks and B. G. Fallone, "Contrast enhancement of portal images by selective histogram equalıztion," Medical Physics20, 199-204(1993).

7 G. Matheron, Random Sets and Integral Geometry (John Wiley \& Sons, New York, 1975).

8 J. Serra, Image Analysis and Mathematical Morphology (Academic Press, London, UK 1982), 2nd edition. 
9 S. R. Sternberg, "Grayscale morphology," Computer Vision, Graphics and Image Processing 35 333-355 (1986).

10 R. M. Haralick, S. R. Sternberg, and X. Zhuang, "Image analysis using mathematical morphology," IEEE Transactions on Pattern Analysis and Machine Intelligence 9. $532-550$ (1987).

11 E. R. Dougherty and C. R. Giardina, Matrix Structured Image Processing(PrenticeHall, New Jersey ,1987).

12 C. R. Giardina and E. R. Dougherty, Morphological Methods in Image Processing (Prentice-Hall, New Jersey, 1988).

13 L. G. Roberts, "Machine perception of three dimensional solids," Optical and Electrooptical Information Processing (editors: B. S. Lipkin and A. Rosenfeld, Camgridge, Massachusetts, 1965).

14 J. M. S. Prewitt, "Object enhancement and extraction,"Picture Processing and Psychopictorics (B. S. Lipkin and A. Rosenfeld editors, Academic Press, New York , 1970) 20, 99-102.

15 L. S. Davis, "A survey of edge detection techniques," Computer Graphics and Image Processing 4, 248-270 (1975).

16 D. Marr and E. Hildreth, "Theory of edge detection," Proceedi igs of Royal Society of London B 207, 187-217 (1980). 
17 J. F. Canny, "A computational approach to edge detection," IEEE Transactions on Pattern Analysis and Machine Intelligence 8, 679-698 (1986).

!: R. Deriche, "Using Canny's criteria to derive a recursively implemented optimal edge detector," International Journal oi Computer Vision 1, 167-187 (1987).

19 J. S. J. Lee, R. M. Haralick, and L. G. Shapiro, "Morphological edge detection," Proceedings of 8th International Conference on Pattern Recognition, 369-373 (1986).

${ }^{20}$ K. Leszczynski, S. Shalev, and G. Gluhchev, "Verification of radiotherapy treatments: Computerized analysis of the size and shape of radiation fields," Medical Physics 20, 687-694 (19:3).

21 W. Press, S. Teukolshy, W. Vetterling, and B. Flannery,Numerical Recipes in C, 2nd edition (Cambridge University Press, Cambridge, UK, 1992).J. M. Balter, C. A. Pelizzari, and G. T. Y. Chen, "Correlation of projection radiographs in radiation therapy using open curve segments and points," Medical Physics 19, 329-334 (1992). 


\section{Chapter 5}

\section{Matching Radiation Fields}

$\S 5.1 \quad$ Introduction $\ldots \ldots \ldots \ldots \ldots \ldots \ldots \ldots \ldots \ldots \ldots$

$\S 5.2 \quad$ Materials and Methods $\ldots \ldots \ldots \ldots \ldots \ldots \ldots \ldots \ldots$

$\S \S 5.2 .1 \quad$ Edge Distance Map Generation . . . . . . . . . 152

$\S \S 5.2 .2$ Cost Function Minimization $\ldots \ldots \ldots \ldots \ldots \ldots$

$\S 5.3 \quad$ Results and Discussions $\ldots \ldots \ldots \ldots \ldots$. . . . . . . 157

$\S \S 5.3 .1 \quad$ Minimization Approach $\ldots \ldots \ldots \ldots \ldots \ldots \ldots$

$\S \S 5.3 .2 \quad$ Computation Speed . . . . . . . . . . . . . . 162

$\S \S 5.3 .3 \quad$ Effects of Cost Functions $\ldots \ldots \ldots \ldots \ldots \ldots 2$

$\S \S 5.3 .4$ Effects of Distance Transformations . . . . . . . . 164 


\section{\$ 5.1 Introduction}

With the ongoing improvement in treatment planning accuracy, there is greater demand on the accurate implementation of a treatment plan. A significant amount of research has been carried out on portal-reference image registration procedures. ${ }^{1-6}$ Current progress in this relatively new field and its impact on clinical practice of radiation therapy have been sys!ematically reviewed. ${ }^{7,8}$

To set up a radiation therapy treatment, one needs to register portal images to a reference image in order to visualize the coverage of the target by the radiation beam. Because of the radiation required for portal image acquisition, it is not feasible to perform beam shaping and localization at the same time. The common practice is to shape the radiation beam by following the prescription before setting the patient up, then to direct the shaped beam to the desired target by positioning the patient. Accurate beam shaping is not only a factor determining the precision of beam coverage but is also beneficial for the interactive beam localization in this two step procedure. Extracting anatomical landmarks for image registration is time consuming. On the other hand, the radiation field is a prominent feature in a portal image. A correctly shaped field can be automatically extracted and used as a registration landmark. Computer programs that can automatically align the portal and reference image to the field and show the relative position of the target in the field can serve as a tool for beam localization with an on-line imager. In 
this chapter, we propose a simplified method for aligning radiation fields.

A natural way to detect shaping errors is to align the prescribed field with the treatment field, and to evaluate the visual match. Among all the techniques that have been employed in this task, chamfer matching is probably the most successful one due to its insensitivity to noise and to discrepancies in shapes. A technical issue in chamfer matching is the minimization of a cost function of the geometric transformation parameters (translation offsets, rntation angle and scaling factor). Since there is no analytical expression for a cost function, the minimization has to rely on iterative searching techniques among which the downhill simplex ${ }^{9}, 10$ and the Powell's ${ }^{10}$ methods has been used by Gilhuijs and van Herk ${ }^{11}$, and by Leszczynski et al ${ }^{12}{ }^{13}$, resp̀ectively. In a recent study on multileaf collimator configuration verification, Zhou and Verhey ${ }^{14}$ reported that the downhill simplex method is not very sensitive to rotation and requires starting points close to the global minimum. To overcome these shortcomings, they used Hough transform and geometric properties of two contours to determine the starting point of chamfer matching. The downhill simplex method is stroightforward and easy to implement but not very efficient in terms of the number of function evaluations that it requires. Powell's method is almost surely faster in all likely applications. ${ }^{10}$ If chamfer matching is used in combination with lower order geometric moments, the minimization process will start very close to the global minimum. ${ }^{15}$ Because of this, 
standard minimization techniques which are designed for general purposes could be simplified to suit specific applications. In this chapter, we propose a simplified adaptation of the chamfer technique for matching treatment and prescription field pairs.

\section{§.2 Materials and Methods}

Thirty pairs of simulation and double-exposure portal films randomly selected from our patient archives were digitized to $512 \times 512$ digital images with 8 bit contrast. The prescribed field contours were drawn with a mouse by following the prescription on the simulation images. The treatment field contours were obtained by using a "contour" operation on the field masks sutomatically extracted from the portal images. ${ }^{5}$ The "contour" operation peels the mask (an object in a binary image) at the depth of one pixel with an "erosion" operation and then subtract the eroded mask from the original one to acquire the field contour.

\section{$\S \S$ 5.2.1 Edge Distance Map Generation}

An edge distance map image $E(\bullet, j)$ (Figure 5.1c) was generated from a binary image containing a prescribed field contour (Figure 5.1a). The value of a pixel in the edge map image is the distance from that pixel to the closest feature (contour) pixel. The higher the value of a pixel, the farther away it is from the contour points. This edge map image, resembling a landscape model with a valley along the prescribed field contour, will be used as a mould onto which the treatment field contour (Figure 5.1b) will be fit. 
By analogy, the treatment field contour will have different gravitational potential energy at different locations and with different orientations (Figure 5.1d). Registration will be achieved when the treatment field contour slides down into the valley under the action of gravity.

Ideally, the value of a pixel in an edge map image should be the Euclidean distance from that pixel to the closest contour point. However calculating Euclidean distances is computationally intensive and yet may not be wurthwhile because of digitization effect on the contours. The common approach to generate an edge map is to use distance transformation which approximates global distances by propagating local distances. ${ }^{16} \mathrm{By}$ analogy, local distance propagation is similar to using a ruler of unit length for measuring long distances. As the ruler is being passed in steps, the number of passes is counted and the total number of counts is used as the distance from the starting point. A distance transformation passes a kernel which carties distances between a pixel and its neighbors over an image and assigns the accumulated distances to the pixels it passes by.

Our edge distance maps were generated with the "chessboard" distance transformation ${ }^{17}$ which employes the $3 \times 3$ kernel

$$
\left[\begin{array}{lll}
1 & 1 & 1 \\
1 & 0 & 1 \\
1 & 1 & 1
\end{array}\right]
$$

for representing the local distances and propagates the local distances in two steps. In 


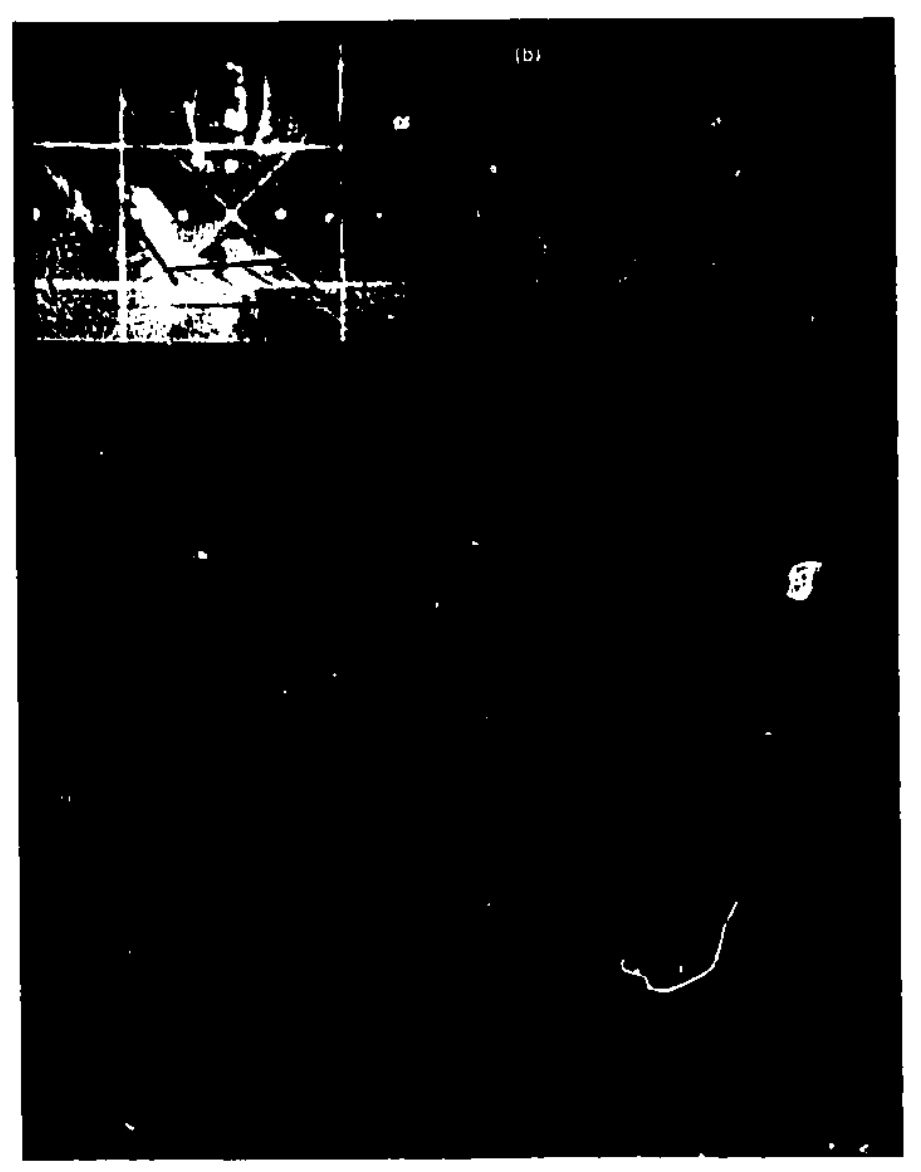

Figure 5.1. Illustration of procedure for chamfer matching: (a) Simulator image; (b) Portal image; (c) Prescribed field contour obtained from (a); (d) Treatment field coriour extracted from (a); (e) Edge distance map generated from (c); (f) After (d) is transformed with a trial set of parameters, it is overlaid on top of (e). 
the first step, the upper-left half of the "chessboard" kernel

$$
\left[\begin{array}{lll}
1 & 1 & 1 \\
1 & 0 &
\end{array}\right]
$$

was passed over the contour image (in which all the contour pixels were assigned the value of 0 and all the non-contour pixels were assigned the value of $\infty$ ) from left to right and from top to bottom. At each pixel, this half kernel was added to that pixel and its four neighbors and the minimum value among the five was assigned to the corresponding pixel in an intermediate image. In the second step, the lower-right half of the "chessboard" kernel

$$
\left[\begin{array}{lll} 
& 0 & 1 \\
1 & 1 & 1
\end{array}\right]
$$

was passed over the intermediate image from right to left and from bottom to top. The minimum value among the five was assigned to the corresponding pixel in the distance image.

\section{$\S \S$ 5.2.2 Cost Function Minimization}

After the edge map (Figure 5.1c) was generated from the prescribed field contour (Figure 5.1u), a geometric transfornution with a set of trial parameters, where $(a, b)$ is the translation vector, $\theta$ is the rotation angle around the center of mass of the prescribed field, $\left(X_{p}, Y_{p}\right)$, and $m$ is the scaling factor, was applied to the treatment field contour $\left\{\left(x_{i}, y_{i}\right) ; i=1,2, \cdots N\right\}$ (Figure 5.1b). The transformed contour $\left\{\left(x_{i}^{\prime}, y_{i}^{\prime}\right) ; i=1,2, \cdots N\right\}$ 
where

$$
\left[\begin{array}{c}
x_{i}^{\prime}-X_{p} \\
y_{i}^{\prime}-Y_{p}
\end{array}\right]=\left[\begin{array}{cc}
m \cos \theta & -m \sin \theta \\
m \sin \theta & m \cos \theta
\end{array}\right]\left[\begin{array}{c}
x_{i}-X_{p} \\
y_{i}-Y_{p}
\end{array}\right]+\left[\begin{array}{l}
a \\
b
\end{array}\right]
$$

was overlaid on the edge map (Figure 1d), and the sum of the values of the pixels in the edge map along the transformed treatment field contour was used as the cost function

$$
F(a, b, \theta, m)=\sum_{i=1}^{N} E\left(x_{i}^{\prime}, y_{i}^{\prime}\right)
$$

$F(a, b, 0, m)$ is a function of the transformation parameters and has its minimum value when the correct transformation parameters relating treatment field contour to the prescribed one are used. Minimization of this cost function is usually achieved with iterative searching algorithms which start with a set of the initial trial parameters $\left(a_{0}, b_{0}, \theta_{0}, m_{0}\right)$, navigate in the 4 dimensional space formed by all the possible transformation parameters, and check some criterion at each step until convergence is reached.

We used a method similar to that used by Zhou and Verhey to obtain the starting point. Except for the rotation angle (which is set as 0 ), the initial trial transformation parameters are obtained from the center of mass and the area of the fields:

$$
\left(a_{0}, b_{0}, \theta_{0}, m_{0}\right)=\left(X_{p}-X_{\ell}, Y_{p}-Y_{l}, 0, \sqrt{\frac{A_{p}}{A_{\ell}}}\right)
$$

where $\left(X_{p}, Y_{p}\right)$ and $A_{p}$ are the center of mass and area of the prescribed field, $\left(X_{t}, Y_{t}\right)$ and $A_{t}$ are those of the treatment field. $\theta_{0}$ is set to 0 because the angle between the two contours is always very small before matching in our case. 
After this preliminary matching, the starting point is very close to the global minimum of the cost function. We minimize the cost function with respect to one variable at a time, one after another and cycle after cycle. One dimensional minimization is achieved by bracketing (Appendix $\mathrm{A}$ ).

The cost function is minimized with respect to 0 first. The initial bracket is set to $\left(\theta_{0}, \theta_{0}+0.1\right)$. The bracketing stops when $\left|\frac{F\left(a_{0}, b_{0}, \theta_{n,}, m_{0}\right)-F\left(a_{0}, b_{0}, \theta_{n+1}, m_{0}\right)}{F\left(a_{0}, b_{0}, \theta_{n+1}, m_{0}\right)}\right| \leq 10^{-1}$ where $n$ is the number of iteration. Similar procedures are then carried out to $a, b$ and $m$ sequentially with initial brackets $\left(a_{0}, a_{0}+5\right),\left(b_{0}, b_{0}+5\right)$ and $\left(m_{0}, m_{0}+0.1\right)$, respectively. All the procedures are stopped at the same precision. Once a bracketing procedure stops, the corresponding variable is kept at the convergent value. Since our stopping criterion is set very low, one cycle is sufficient to reach convergence for all the variables.

\section{§ 5.3 Results and Discussions}

Chamfer matching is a technique of patten recognition type. The goodness of a match is characterized by a similarity measure. The measure we used is the average edge distance which is the minimum cost. It is defined as the average pixel value along the registered treatment field contour in the edge map image, which can be expressed as $\frac{1}{N} F\left(a_{m}, b_{m}, \theta_{m}, m_{m},\right)$ where $N$ is the number of pixels along the treatment field contour and $\left(a_{m}, b_{m}, \partial_{m}, m_{m}\right)$ is the final transformation parameters reached by the minimization. 
If the two feature being matched are perfectly similar to each other, the minimum cost should be zero. Otherwise, it will take on a positive value. When matching simple features like closed field contours, unless the shaping errors are extremely large, a smaller minimum cost should correspond to a better match.

\section{$\S \S$ 5.3.1 Minimization Approach}

To test the viability of successive minimizations along coordinate directions in chamfer matching when combined with geometric moments, we compared the average edge distance obtained with successive mirimization along coordinate directions, $D_{\text {S.M.A.C.D. }}$ to that obtained with the downhill simplex method $D_{\text {simplex }}$. A flowchart of the comparison test is shown in Figure 5.2.

Throughout the comparison tests, both minimization procedures are started after the first step match obtained with geometric moments in Eq. 5.6. The vertices of the initial simplex are set to

$$
\begin{gathered}
\left(a_{0}, b_{0}, \theta_{0}, m_{0}\right) \\
\left(a_{0}+5, b_{0}, \theta_{0}, m_{0}\right) \\
\left(a_{0}, b_{c}+5, \theta_{0}, m_{0}\right) \\
\left(a_{0}, b_{0}, \theta_{0}+0.1, m_{0}\right) \\
\left(a_{0}, b_{0}, \theta_{0}, m+0.1\right)
\end{gathered}
$$

where $a_{0}, b_{0}, \theta_{0}$ and $m_{0}$ are given in Eq. (5.6). The absolute values of the minimum costs obtained with the two methods are very close. In order to see the difference clearly, we 


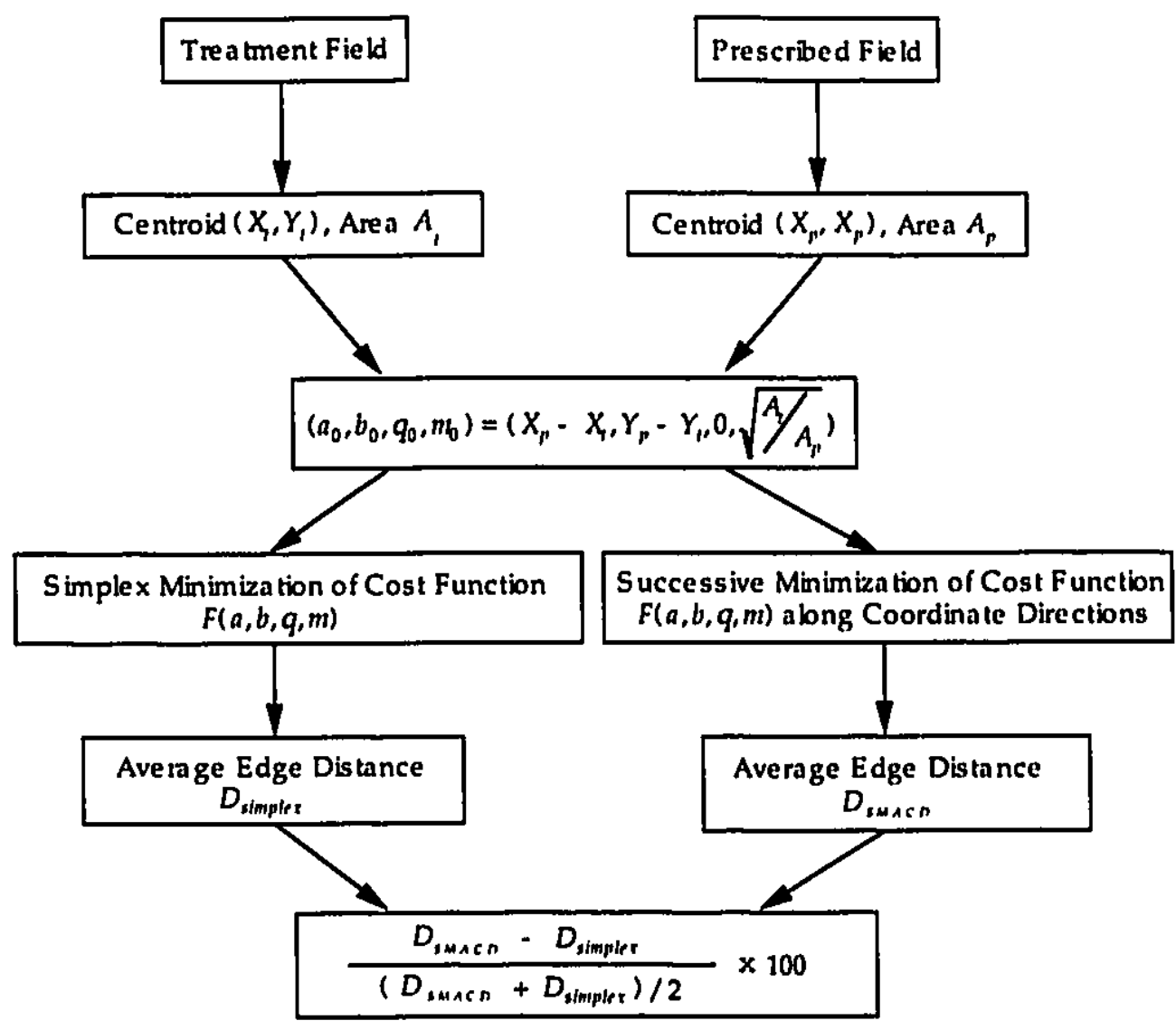

Figure 5.2. Flowchart of the comparison test between the simplex method and successive minimization along coordinate directions (S.M.A.C.D). 
calculated the relative difference in percentage

$$
\frac{D_{\text {S.M.A.C.D. }}-D_{\text {simplex }}}{\left(D_{\text {S.M.A.C.D. }}+D_{\text {simplex }}\right) / 2} \times 100,
$$

a histogram of which obtained from the 30 cases is plotted in Figure 5.3a.

It can be seen that, on average, successive line minimization along coordinate directions can achieve a smaller residual value of the cost function therefore has better precision than the simplex algorithm in this matching scheme.

Successive minimization along coordinate directions is actually the first step of the Powell's method. ${ }^{10}$ Starting from the coordinate direction set, Powell's method successively minimizes a function in each direction in the set and adjusts the direction set after each cycle of line minimization through all the variables. The adjustment of searching directions is to handle functions which can not be well approximated by quadratic forms because Powell's method is based on Taylor expansion. For example, a function having a long narrow valley will make successive minimization along coordinate directions very inefficient. However, when simple geometric objects like the field contours, are being matched, it is very unlikely that the cost function will have such erratic behavior. Moreover, after prelininary matching has been done with geometric moments, the starting point is very close to the global minimum of the cost function. In this neighborhood, the cost function can be well approximated by a quadratic form which can be exactly minimized by one pass of line minimization through all the variables. 

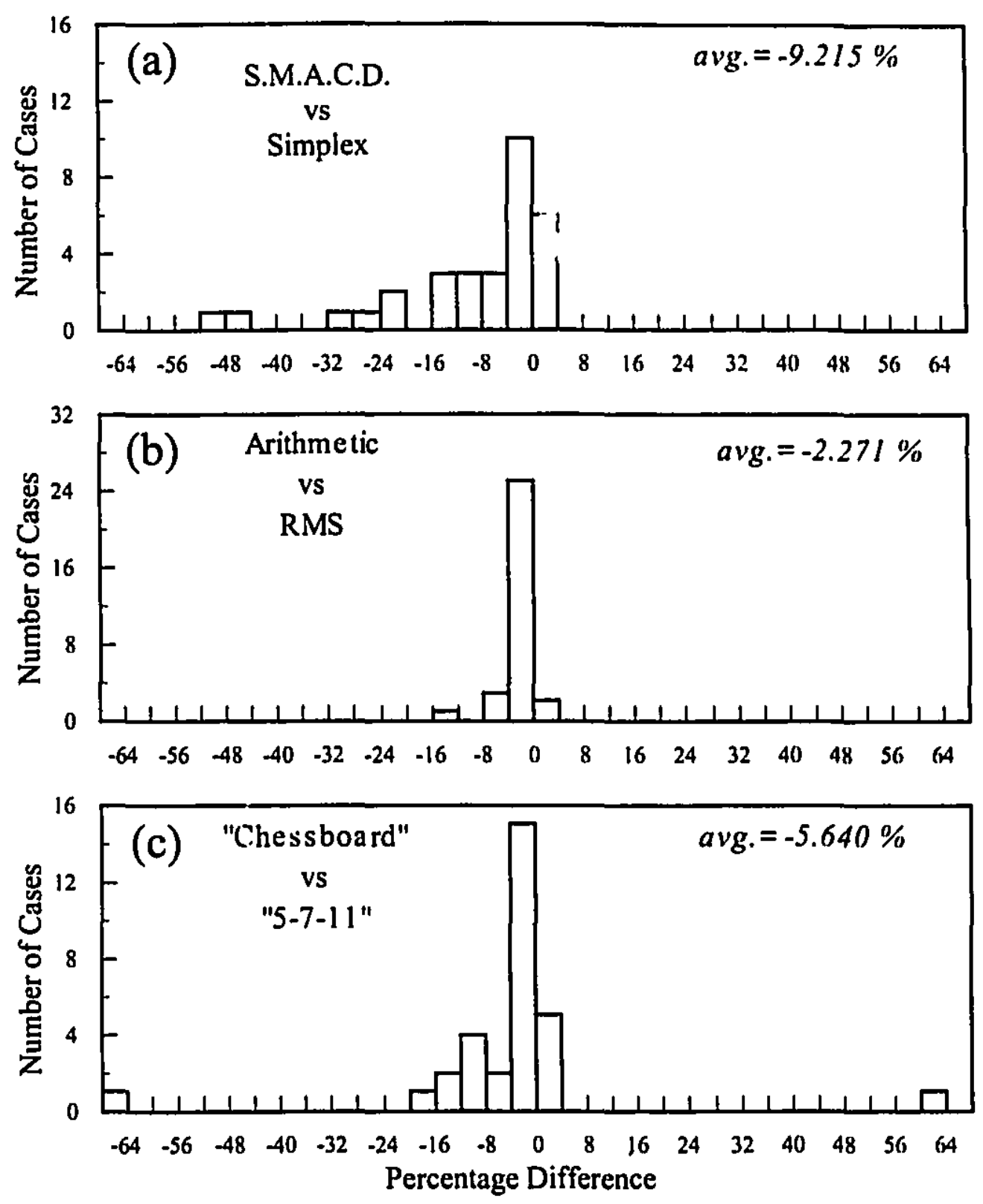

Figure 5.3. Comparison of final average edge distances achieved with different approaches. (a) successive line minimization versus the simplex method with "chessboard" edge distancs maps and arithmetic average edge distance as cost function. (b) arithmetic versus root of mean square average edge distance as cost function when "chessboard" edge maps and successive line minimization were used. (c) "chessboard" versus "5-7-11" edge maps when arithmetic average edge distance as cost function was minimized successive line minimization. 


\section{$\S \S$ 5.3.2 Computation Speed}

The two algorithms are also compared in term of computation speed. Table 5.7 lists the numbers of iterations to reach convergence. Convergence is defined in the following manner: for successive line minimization, either the precision criterion is satisfied or the values of the cost function at the two ends of the bracket no longer change; for simplex algorithm, either the precision criterion is satisfied or the values of the cost function at the vertices of the simplex no long change. It should be noticed that not only less number of iterations are required for sequential bracketing ( 3.2 on the average), but the number of calculations involved in a single iteration in bracketing is also much less than that in the simplex algorithm. The reason we used the numbers of iterations to measure computation speed is that the absolute computation time for either algorithm on our computer (Indigo, Silicon Graphics Inc., Mountain View, California) is actually very short since the search starts from close to the minimum cost. This comparison is to show that the better accuracy of successive minimization along coordinate directions as discussed previously is achieved without sacrificing computation speed.

\section{$\S \S$ 5.3.3 Effects of Cost Functions}

According to the investigation by Borgefors, ${ }^{18}$ the cost function based on the root of mean square average edge distance (summing up the square of the pixel value along the contour that is being matched when it is fitted into the edge map) has fewer local 
Table 5.7 Numbers of iterations for reaching convergence in successive line minimization along coordinate directions $\left(N_{S . M . A . C . D .}\right)$ and the downhill simplex method $\left(N_{\text {simples }}\right)$. The average difference $\overline{\left(N_{\text {S.M.A.C.D. }}-\bar{N}_{\text {simples }}\right)}=-3.2$.

\begin{tabular}{|c|c|c|c|}
\hline case number & NS.AT.A.C.D. & Ninngles & Ns.nacen-Numplot \\
\hline 1 & 32 & 31 & 1 \\
\hline 2 & 31 & 24 & 7 \\
\hline 3 & 33 & 41 & .8 \\
\hline 4 & 32 & 40 & -8 \\
\hline 5 & 25 & 24 & $\mathbf{l}$ \\
\hline 6 & 30 & 38 & -8 \\
\hline 7 & 35 & 47 & .12 \\
\hline 8 & 34 & 47 & .13 \\
\hline 9 & 24 & 33 & .9 \\
\hline 10 & 32 & 31 & 1 \\
\hline 11 & 29 & 44 & .15 \\
\hline 12 & 29 & 19 & 10 \\
\hline 13 & 34 & 34 & $\mathbf{0}$ \\
\hline 14 & 32 & 35 & -3 \\
\hline 15 & 34 & 42 & -8 \\
\hline 16 & 28 & 25 & 3 \\
\hline 17 & 36 & 44 & -8 \\
\hline 18 & 36 & 37 & -1 \\
\hline 19 & 32 & 34 & .2 \\
\hline 20 & 30 & 32 & .2 \\
\hline 21 & 33 & 33 & 0 \\
\hline 22 & 23 & 33 & -10 \\
\hline 23 & 29 & 32 & .3 \\
\hline 24 & 26 & 23 & 3 \\
\hline 25 & 31 & 37 & .6 \\
\hline 26 & 32 & 38 & .6 \\
\hline 27 & 30 & 31 & .1 \\
\hline 28 & 26 & 26 & 0 \\
\hline 29 & 31 & 29 & 2 \\
\hline 30 & 31 & 32 & .1 \\
\hline
\end{tabular}


minima than does the cost function based on the arihmetic average edge distance. It can, therefore, reduce the chance for minimization being trapped by false convergence. Local minima are not a problem within our approach since they are bypassed by the first approximation [Eq.(5.6)]. To investigate the effect of the two types of cost function in this application, we calculated the average edge distance, and observed a slight advantage of the arithmetic average type cost function over the one of root of mean square average type (Figure 5.3b). In chamfer matching every point on the treatment field contour contributes to the cost function. The root of mean square average type cost function increases faster around the minimum and therefore assigns more weight to the distorted parts of the treatment field contour making the minimum of the cost function not correspond as well to the actual match as does the arithmetic average type cost function.

\section{$\S \S$ 5.3.4 Effects of Distance Transformations}

The accuracy of chamfer matching also depends on the distance transformation used to generate the edge distance map. The difference in the edge distance map will be carried into the cost function. The "chessboard" distance transformation is one of the simplest approximations of the Euclidean Cistance transformation because it assigns the sarne distance from a pixel to all its 8 immediate neighbors while the Euclidean distance from a pixel to its diagonal neighbor is $\sqrt{2}$. Closer approximations can be achieved by (a) assigning different numbers to the orthogonal and the diagonal neighbors that better 
represent the $1: \sqrt{2}$ ratio, e.g. the $3 \times 3$ kernel in the "3-4" distance transformation"

$$
\left[\begin{array}{lll}
4 & 3 & 4 \\
3 & 0 & 3 \\
4 & 3 & 4
\end{array}\right]
$$

or by (b) using a larger kernel to take more neighbors into account, such as the "5-7-11" kernel $^{16}$

$$
\left[\begin{array}{ccccc}
14 & 11 & 10 & 11 & 14 \\
11 & 7 & 5 & 7 & 11 \\
10 & 5 & 0 & 5 & 10 \\
11 & 7 & 5 & 7 & 11 \\
14 & 11 & 10 & 11 & 14
\end{array}\right] .
$$

The deviation from the true Euclidean gauge may also result in local minima of the cost function when minimization starts far from the global minimum. Except for orientation, the treatment field contour in our case has been brought very close to the matched position by the first approximation [Eq.(5.6)]. To determine whether a closer approximation to the Euclidean distance transformation would have an advantage in our case, we compared the effect of the "5-7-11" kernel with that of the "chessboard" kumel. The average edge distance obtained with the "5-7-11" kernel has been divided by 5 to normalize the unit distance to one. Figure $5.3 \mathrm{c}$ shows that the "chessboard" kernel has a slightly better performance than the "5-.7-11" one. This can be explained rather simply. An edge distance map generated by a distance transformation ("chessboard" or "5-7-11") is very accurate in specifying the orientation of a feature. When the minimization with respect to the rotation angle is completed, the triatment field contour is very close to the final registration. The advantage of the "5-7-11" ketnel only occurs when the searching 
process starts at a point which is far from registration. This situation does no: occur in our case because we start with a relatively close approximation. On the other hand, the slopes in the edge map generated with the "5-7-11" kernel are steeper so that shaping errors and noise on the treatment field coniour will have more weight in the cost function weakening the correspondence of the minimum of the cost function to the actual match.

In conclusion, our results show that incorporating additional geometric informatior. into chamfer technique may improve performance without sacrificing computation speed when matching closed contours. In this kind of tasks, successive line minimization of the cost function along coordinate directions is viable when chamfer matching follows a preliminary alignment. Recently, Lescznynski et al ${ }^{13}$ considered the fact that the treatment field is not a rigid feature because it is shaped with different devices. In order to better locate the shaping errors, they broke the treat:nent field contour into segments and used the chamfer matching technique to match these segments separately. The Powell's algorithm was employed for the minimization of their cost function. The segments of a closed contour are simpler feature than the contour itself. Our method can be easily customized to handle this task. 


\section{References}

1 J. Bijhold, K. G. A. Gilhuijs, M. van Herk and Meertens, "Radiation field edge detection in portal images," Physics in Medicine and Biology 36, 1705-1710 (199.1).

2 W. C. Lam, K. S. Herman, K. S. Lam, and D. J. Lee, "On-line portal imaging: computer-assisted error measurement," Radiology179, 871-873 (1991).

3 S. M. Jones and A. L. Boyer, "Investigation of a FFT-based correlation teclunique for verification of radiation treatment setup," Medical Physics 18, 1116-1125 (1991).

4 J. M. Balter, C. A. Pelizzari, and G. T. Y. Chen, "Correlation of projection radiographs in radiation therapy using open curve segments and points," Medical Physics 19. 329-334 (1992).

5 H. Wang and B. G. Fallone, "A robust morphological algorithm for automatic radiation field-extraction and correlation of portal images," Medical Physics 21, 237-244 (1994).

6 J. Moseley and P. Munro, "A semiautomatic method of registration of portal images," Medical Physics 21, 551-558 (1994).

7 A. L. Boyer, L. Antonuk, A. Fenster, M. van Herk, H. Meertens, P. Munro, L. Reinstein and J. Wong, "A review of electronic portal imaging devices(EPIDs)," Medical Physics 19, 1-16 (1992).

8 M. van Herk, K. Gilhuijs, P. Williams, P. Cinquin, L. Cionini, and W. Swindel, "Iole 
of elecirutic portal imaging in high dose/high precision radiotherapy," Radiotherapy and Oncology 21, 269-270 (1994).

9 J. Nelder and R. Mead, "A simplex method for function minimization," Computer Journal 7, 308-313 (1964).

10 W. Press, S. Teukolshy, W. Vetterling, and B. Flannery,Numerical Recipes in $C$, 2nd edition (Cambridge University Press, Cambridge, UK, 1992).

11 Gilhuijs and M. van Herk, "Automatic on-line inspection of patient setup in radiation therapy using digital portal images," Medical Physics 20, 667-677 (1993).

12 K. Leszczynski, S. Shalev, and G. Gluhchev, "Verification of radiotherapy treatments: Computerized analysis of the size and shape of radiation fields," Medical Physics 20, 687-694 (1993).

13 K. Leszczynski and S. Loose and P. Dunscombe, "Segmented chamfer matching for prescription-treatment image registration in radiotherapy," Physics in Medicine and Biology 40, 83-94 (1995).

14 S. Zhou and L. J. Verhey, "A robust method of multileaf collimator (MLC) leaf configuration verification," Physics in Medicine and Biology 39, 1929-1947 (1994).

$15 \mathrm{H}$. Wang and B. G. Fallone, "A sequential minimization technique in chamfer matching," in Proceedings of 3rd Imternational Workshop On Electronic Portal Imaging (San Francisco, California 1994). 
${ }^{16}$ G. Borgefors, "Distance transformation in digital images," Computer Vision, Graphics and Image Processing 34, 344-371 (1986).

17 A. Rosenfeld and A. D. Kak, Digital Picture Processing (Academic Press, New York, 1982).

18 G. Borgefors, "An improved version of the chamfer matching algorithm," in Proceedings of the 7th International Conference on Pattern Recognition (Montreal, Canada. 1984). 


\section{Chapter 6}

Summary

$\S 6.1 \quad$ Electrostatic Portal Imaging $\ldots \ldots \ldots \ldots \ldots \ldots \ldots 171$

$\$ \S 6.1 .1 \quad$ Amorphous Selenium $\ldots \ldots \ldots \ldots \ldots \ldots \ldots$

$\S \$ 6.1 .2 \quad$ Front Metal Plate $\ldots \ldots \ldots \ldots \ldots \ldots \ldots \ldots$

$\S \S 6.1 .3 \quad$ Future Work $\ldots \ldots \ldots \ldots \ldots \ldots \ldots$

$\S 6.2$ Portal Image Segmentation $\ldots \ldots \ldots \ldots \ldots \ldots \ldots$

$\S 6.3$ Radiation Field Shape Verification . . . . . . . . . 175 


\section{§ 6.1 Electrostatic Portal Imaging}

With the devclopment of novel digital readout methods, xeroradiography is starting to draw more and more interest in the medical imaging community. Although there have been quite a number of publications on the recent progress of this imaging modality, most of the effort has been devoted to xeromammography where low energy $\mathrm{x}$ ray are used for acquiring high quality images. Incited by the advantages of electrostatic imaging in the diagnostic energy range, this thesis intends to explore the possibility of introducing this technique to megavoltage portal imaging which is an essential means to ensure the quality of radiation treatments. The preliminary study of developing such a portal imager has led to some positive conclusions.

\section{§ 6.1.1 Amorphous Selenium}

$\mathrm{X}$-ray sensitivity of the metal/a-Se detector is determined by the metal and the thickness of the a-Se layer. The rate of radiation discharge becomes faster as the a-Se thickness is increased. Detective quantum efficiency increases slightly with the thickness of a-Se because of a more complete absorption of incident $x$-rays. Increasing the thickness of a-Se can slightly degrade spatial resolution. The optimal thickness of a-Se is more likely to determine the proper discharge slope and dynamic range. 


\section{$\S \S$ 6.1.2 Front Metal Plate}

The conventional configuration of a-Se receptors used in diagnostic imaging, i.e., exposing the a-Se directly to the radiation beam transmitted through the patient, cannot be used in portal imaging as the beam has been severely contaminated with scattered radiation from the patient. Having energies lower than those of the primary $x$-rays, the scattered radiation is more likely to interact with the receptor, generate noise signal and consequently smear out the image. A front metal is required to shield the sensitive volume of the receptor from the scattered radiation from the patient. In addition to removing scatter, a front metal plate enhances detector sensitivity to high energy $\mathrm{x}$ rays as it interacts with the primary photons and converts them into secondary electrons which deposit energy and generate signal in the detector. This conversion process leads to a more complete absorption of the energy of primary photons, therefore reducing the fluctuation in the amount of absorbed energy and improving detective quantum efficiency. As for spatial resolution, a front metal plate can reduce detector blur at low energies due to the high density of the metal. Without the metal plate, the lateral spread of the signal would be larger in the build-up region which is made up of a-Se itself. At higher energies, a front metal plate increases detector blur as it increases detector sensitivity. However, this should not be considered as a disadvantage since the metal plate is required for scatter filtering. The modulation transfer function of the receptor appears to be insensitive to 
the type and thickness of the metal plate that we studied.

\section{$\S \S$ 6.1.3 Future Work}

Being a preliminary study, this thesis concentrates on the theoretical aspects of employing a-Se in electrostatic portal imaging. The prototype imager described in this thesis is still in the laboratory stage and is not yet ready to be used for clinical imaging mostly due to the single probe readout system. Currently, it takes about 7 minutes to scan a $6 \times 6$ in $^{2}$ area. A practical readout system needs to be developed to speed up image acquisition to a realistic level. Increasing the number of probes is not likely to satisfy the requirements of on-line imaging. If an array of probes is used to scan the receptor, the speed of the mechanical scan will not be fast enough for real-time acquisition. Using a matrix of probes will make the cost unacceptable. Another method that can be looked into is the photoinduced discharge method used by Rowlands and Hunter ${ }^{1}$ for fast readout of a-Se image receptors in diagnostic imaging. However, this type of readout system is very bulky. It would be very difficult to mount such a system on a linear accelerator. Recently, Zhao and Rowlands ${ }^{2}$ reported a flat-panel device used for real-time readout of electrostatic images. This device consists of a two-dimensional array of thin film transistors and is directly coupled to the image receptor, therefore, is very compact. This device has sufficient resistance to radiation damage in diagnostic imaging. A similar device has been used by Antonuk $e a^{3}$ to replace the traditional mirror-lens system in 
fluoroscopic portal imager and has been reported to be robust to resist radiation damage at therapy energies. This type of device will very likely be the choice for an on-line readout system in a metal/a-Se based portal imager. With a fast readout system, the metal/a-Se combination could become a possible altemative. The potential with our metal/a-Se system lies with the fact that the direct output of the metal/a-Se combination is already charge while the direct output of the phosphor-based systems is light which must be subsequently converted to charge. The light-to-charge conversion introduces processes that may degrade the MTF and the DQE.

Another task that needs to be undertaken is systematic evaluation of the performance of the prototype imager in terms of its spatial resolution and noise level. This can be done both in an absolute nanner, i.e., measuring the MTF and DQE, and in comparison to current commercial imagers, i.e., comparing images of identical phantoms.

\section{§ 6.2 Portal Image Segmentation}

In addition to the prototype portal imager, we have developed a model-based algorithm for automatic segmentation of portal images. The algorithm has been proved to be accurate, robust and fast. These advantages originate from the use of morphological techniques and the model based approach which employes multiple criteria and dynamic reasoning. The algorithm is capable of accommodating a large variation of portal images types and of handling images that are very difficult to segment. When combined with 
contrast enhancement techniques, it can lead to improved visibility of anatomical details in portal images.

\section{§ 6.3 Radiation Field Shape Verification}

Another task that we have undertaken is to adapt the chamfer matching technique to a specific application: radiation field shape verification. We have investigated the possibility of employing a simple approach to minimize the cost function in order to achieve better match. Our results showed that incorporating additional geometric information into chamfer technique may improve performance without sacrificing computation speed when matching simple features. In this kind of tasks, successive line minimization of the cost function along coordinate directions is viable when chamfer matching follows a preliminary alignment. 


\section{References}

I J. A. Rowlands and D. M. Hunter, "X-ray imaging using amorphous selenium: Photoinduced discharge (PID) readout for digital general radiography," Medical Physics 22. 1983-2005 (1995).

2 W. Zhao and J. A. Rowlands, "X-ray imaging using amorphous selenium: Feasibility of a flat panel self-scanned detector for digital radiology," Medical Physics 22. 1595-1604 (1995).

${ }^{3}$ L. E. Antonuk, J. Boudry, W. Huang, D. L. MeShan, E. J. Morto, J. Yorkston, M. J. Longo. and R. Á. Street, "Demonstration of megavoltage and diagnostic $x$-ray imaging with hydrogenated amorphous silicon arrays," Medical Physics 19, 1455-1466 (1992). 


\section{Appendix A}

Minimization by Bracketing

Given a continuous unimodal function $f(x)$ in the region $(a, b)$, the minimum can be reached by simple bracketing with a pair of points $a<x_{i}^{(l)}<b$ and $a<x_{i}^{(h)}<b$, where $f\left(x_{i}^{(l)}\right)<f\left(x_{i}^{(h)}\right)$. Here the superscripts at $l$ and $h$ denote "low" and "high", respectively, and the subscript $i$ is the iteration number. Starting from an initial pair of points $x_{0}^{(l)}$ and $x_{0}^{(h)}$, the function is evaluated at the reflection point of $x_{0}^{(h)}$ about $x_{0}^{(l)}$, which is $x^{*}=x_{0}^{(h)}+2\left(x_{0}^{(l)}-x_{0}^{(h)}\right)$, and the value of the function a: this point $f\left(x^{*}\right)$ is

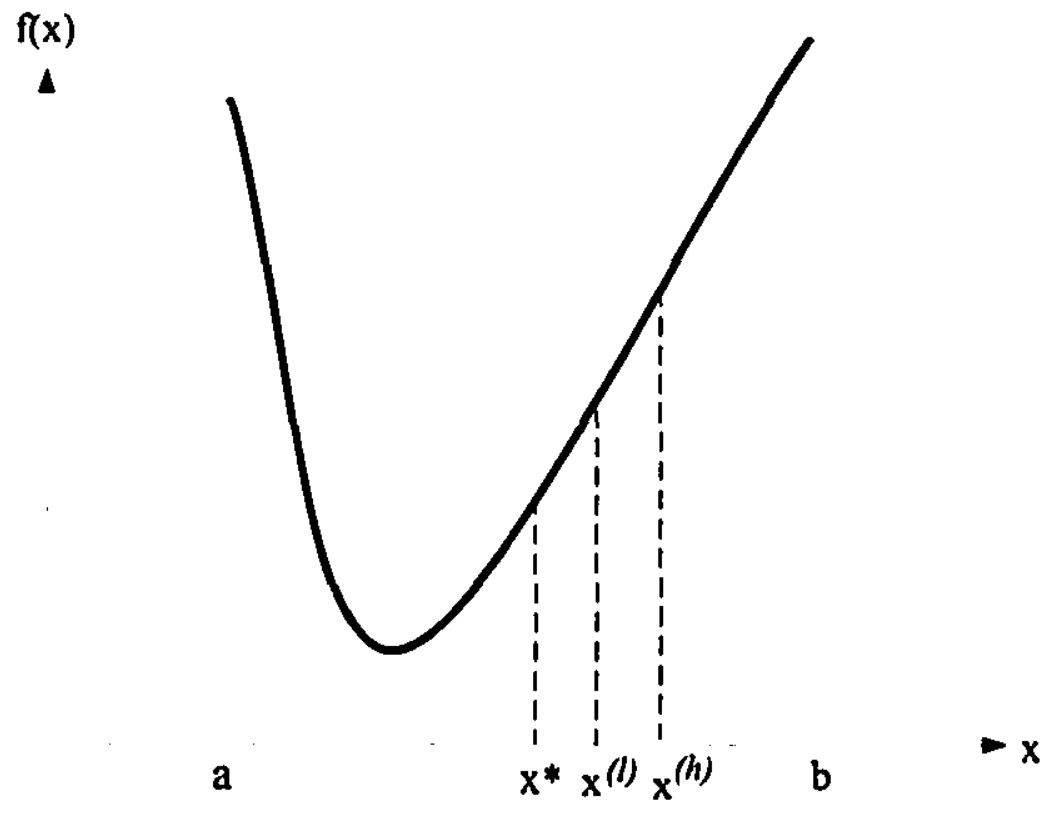

Figure A.1. Illustration of the bracketing process for minimizing a one dimensional function. 
compared with $f\left(x_{0}^{(l)}\right)$ and $f\left(x_{0}^{(h)}\right)$. Then the following assignments are made:

$$
\begin{aligned}
& x_{1}^{(l)}=x^{*} \text { and } x_{1}^{(h)}=x_{0}^{(l)} \text {. if } f\left(x^{*}\right)<f\left(x_{0}^{(l)}\right): \\
& x_{1}^{(l)}=x_{0}^{(l)} \text { and } x_{1}^{(h)}=x^{*} \text {. if } f\left(x_{0}^{(l)}\right) \leq f\left(x^{*}\right)<f\left(x_{0}^{(h)}\right): \\
& x_{1}^{(l)}=x_{0}^{(l)} \text { and } x_{1}^{(h)}=x_{0}^{(l)}+\frac{1}{2}\left(x_{0}^{(h)}-x_{0}^{(l)}\right) \text {. if } f\left(x_{0}^{(h)}\right) \leq f\left(x^{*}\right) ;
\end{aligned}
$$

A precision value $\epsilon$ is preset at $10^{4}$ for our calculations, and if $\left|\frac{f\left(s_{1}^{(\prime)}\right)-f\left(s_{1}^{(h)}\right)}{f\left(s_{1}^{(1)}\right)}\right| \leq c$, the process stops; otherwise the process continues with another iteration. Tile programing syntax is shown below:

ith iteration

if $\left|\frac{f\left(s_{1}^{(t)}\right)-f\left(s_{1}^{(h)}\right)}{f\left(f_{1}^{(l)}\right)}\right| \leq \epsilon$, then $x_{\min }=x_{i}^{(l)}$ and terminate;

else, $i+1$ th iteration

$x^{*}=x_{i}^{(h)}+2\left(x_{i}^{(!)}-x_{i}^{(h)}\right)$

if $f\left(x^{*}\right)<f\left(x_{i}^{(l)}\right)$, then $x_{i+1}^{(l)}=x^{*}, x_{i+1}^{(h)}=x_{i}^{(l)}$;

else if $f\left(x_{i}^{(l)}\right) \leq f\left(x^{*}\right)<f\left(x_{i}^{(h)}\right)$, then $x_{i+1}^{(l)}=x_{i}^{(l)} \cdot x_{i+1}^{(h)}=x^{*}$;

else, then $x_{i+1}^{(l)}=x_{i}^{(l)}, x_{i+1}^{(h)}=x_{i}^{(h)}+\frac{1}{2}\left(x_{i}^{(l)}-x_{i}^{(h)}\right)$;

if $\left|\frac{f\left(s_{1+1}^{(l)}\right)-f\left(x_{1+1}^{(1)}\right)}{f\left(x_{1+1}^{(1)}\right)}\right| \leq \epsilon$, then $x_{\min }=x_{i}^{(l)}$ and terminate;

else, $i+2$ th iteration. 


\section{Bibliography}

E. Antonuk, J. Yorkston, J. Boudry, M. Longo, and R. A. Street, "Large area amorphous silicon photodiode arrays for radiotherapy and diagnostic imaging," Nuclear Instrumentation and Method A310, 460-464 (1989) [5].

E. Antonuk, J. Yorkston, J. Boudry, M. Longo, J. Jimenez, and R. A. Street, "Development of hydrogenated amorphous silicon sensors for high energy photon radiotherapy imaging," Medical Physics NS-37(2), 165-170 (1990) [5].

L. E. Antonuk, J. Boudry, W. Huang, D. L. McShan, E. J. Morto, J. Yorkston, M. J. Longo, and R. A. Street, "Demonstration of megavoltage and diagnostic x-ray imaging with hydrogenated amorphous silicon arrays," Medical Physics 19, 1455-1466 (1992) [173].

N. Baily, R. Horn, and T. Kampp, "Fluoroscopic visualization of megavoltage therapeutic X-ray beams," Int. J. Radiat. Oncol. Biol. Phys. 6, 935-939 (1980) [4].

Y. Bao, "A novel histogram modification approach for medical image enhancement," SPIE 2167, 755-765 (1994) [9].

J. M. Balter, C. A. Pelizzari, and G. T. Y. Chen, "Correlation of projection radiographs in radiation therapy using open curve segments and points," Medical Physics 19, 329-334 (1992) $[9,150]$. 
A. F. Bielajew and D. W. O. Rogers, "PRESTA-the parameter reduced electron-step transport algorithm for electron monte carlo transport." Nuclear Instruments and Method B18, 535-548 (1984) [30].

J. Bijhold, K. G. A. Gilhuijs, M. van Herk and Meertens, "Radiation field edge detection in portal images," Physics in Medicine and Biology 36, 1705-1710 (1991) $[96,150]$.

J. W. Boag, "Xeroradiography," Physics in Medicine and Biology 18, 3-37 (1973) $[7,59]$.

G. Borgefors, "An improved version of the chamfer matching algorithm." in Proceedings of the 7th International Conference on Pattern Recognition (Montreal, Canada, 1984) [162].

G. Borgefors, "Distance transformation in digital images," Computer Vision, Graphics and Image Processing 34, 344-371 (1986) [153,163].

A. L. Boyer, L. Antonuk, A. Fenster, M. van Herk, H. Meertens, P. Munro, L. Reinstein and J. Wong, "A review of electronic portal imaging devices(EPIDs)," Medical Physics 19, $1-16(1992)[4,150]$.

I. Brodie and R. A. Gutcheck, "Radiographic information theory and application to mammography," Medical Physics 9, 79-94 (1982) [7,59].

I. Brodie and R. A. Gutcheck, "Minimum exposure estimates for information recording in diagnostic radiology," Medical Physics 12, 362-367 (1985) [7,59]. 
J. F. Canny, "A computational approach to edge detection," IEEE Transactions on Pattern Analysis and Machine Intelligence 8, 679-698 (1986) [120].

E. L. Cuok, J. D. Edwards, O. L. Nelson and J. E. Potts, "Performance of a high resolution radiographic detector," in The society of Imaging Science and Technology 47th Annual Conference ICPS, 669 (1994) [7,59].

I. Crooks and B. G. Fallone, "PC-based selective histogram equalization for contrast enhancement of portal films," Medical Physics (abstract) 18, 618 (1991) [98].

I. Crooks and B. G. Fallone, "Automatic segmentation of portal images for selective histogram equalization," Medical Physics (abstract) 19, 882 (1992) [98].

1. Crooks and B. G. Fallone, "Contrast enhancement of portal images by selective histogram equalization," Medical Physics 20, 199-204(1993) [9,98].

L. S. Davis, "A survey of edge detection techniques," Computer Graphics and Image Processing 4, 248-270 (1975) [118].

R. Deriche, "Using Canny's criteria to derive a recursively implemented optimal edge detector," International Journal of Computer Vision 1, 167-187 (1987) [122].

C. E. Jick and J. W. Motz, "Image information transfer properties of x-ray image intensifiers," Medical Physics 10, 337-346 (1981) [21].

G. X. Ding, S. Shalev and (J. Gluchev, "A $\rho-\theta$ technique for treatment verification in radiotherapy and its clinical applications," Medical Physics 20, 1135-1143 (1993) [9]. 
L. Dong and A. L. Boyer, "An objective miethod for evaluating electronic portal imaging devices," Medical Physics 21, 755-760 (1994) [86].

E. R. Dougherty and C. R. Giardina, Marrix Structured Image Processing(Prentice-Hall, New Jersey ,1987) [103].

R. T. Droege and B. Bjarngard, "Influence of metal screens on contrast in megavoltage x-ray imaging," Medical Physics 6, 515-518 (1979) [3,23].

P. B. Dunscombe and K. Fox. "The precision of determining compliance with prescribed fields from conventional portal films," British Journai of Radiology 62, 48-52 (1989) [3]. A. Ezz, P. Munro, A. T. Porter, J. Battista, D. A. Jaffray, A. Fenster, and S. Osbome, "Daily monitoring and correction of radiation field placement using a video-based portal imaging system: a pilot study," Int. J. Radiat. Oncol. Biol. Phys. 22, 159-165 (1992) [4].

B. G. Fallone, T. Falco, H. Wang, and N. Araj, "An electrostatic-based detector for portal imaging," in The 4th International Workshop on Electronic Portal Imaging: Portal Imaging in Theory and Practice (Amsterdam, Holland, 1996) [10,23].

B. G. Fallone and T. Falco, "Megavoltage imaging methed using a combination of a photoreceptor with a high energy photon converter and intensifier," International Patent File $(1996)[8,23]$.

D. S. Fritsch, E. L. Chaney, A. Boxwala, M. J. McAuliffe, S. Raghavan, A. Thall, 
and J. R. D. Eamhart, "Core-based portal image registration for automatic radiotherapy treatment verification," Int. J. Radiat. Oncol. 29, 1287-1300 (1995) [9].

C. R. Giardina and E. R. Dougherty, Morphological Methods in Image Processing (Prentice-Hall, New Jersey, 1988) [103,123,144].

Gilhuijs and M. van Herk, "Automatic on-line inspection of patient setup in radiation therapy using digital portal images," Medical Physics 20, 667-677 (1993) [151].

R. M. Haralick, S. R. Sternberg, and X. Zhuang, "Image analysis using mathenatical morphology," IEEE Transactions on Pattern Analysis and Machine Intelligence 9. $532-550(1987)[103]$.

H. F. Hare, J. Hale, and E. P. Pendergrass, "Physical and clinical aspects of supervoltage rotational therapy," Radiology 57, 157 (1951) [2].

D. H. Hristov and B. G. Fallone, "A grey-level image alignment algorithm for registration of portal images and digitally reconstructed radiographs," Medical Physics 23, $75-84$ (1996) [9].

D. A. Jaffray, J. J. Battista, A. Fenster and P. Munro, "Monte Carlo studies of Xray energy absroption and quantum noise in megavoltage transmission radiography," Medical Physics 22, 1077-1088 (1995) [23,30].

D. A. Jaffray, K. Chawla, C. Yu, and J. W. Wong, "Dual beam imaging for online verification of radiotherapy field place.nent," Int. J. Radiat. Oncol. Biol. Phys. 33, 
$1273-1280(1995)[4]$.

H. E. Johns and J. R. Cunningham. The Phisics of Radiolognt thedition, Springficld. Illinois 1983) [84].

S. M. Jones and A. L. Boyer, "Investigation of a FFT-based correlation technique for verification of radiation treatment setup." Medical Physics 18, 1116-1125 (1991) $[9,150]$.

J. J. Kinzie, G. E. Hanks, C. J. Maclean, and S. Kramer, "Patterns of care study: Hodgkin's disease relapse rates and adequacy of portals," Cancer 52, 2223-2226 (1983) [9].

W. C. Lam, K. S. Herman, K. S. Lam, and D. J. Lee, "Or-line portal imaging: computerassisted error measurement," Radiology 179, 871-873 (1991) [150].

J. S. J. Lee, R. M. Haralic!, and L. G. Shapiro, "Morphological edge detection," Proceedings of 8th International Conference on Pattern Recognition, 369-373 (1986).

J. Leong, "Use of digital fluoroscopy as an on-line verification device in radiation therapy," Physics of Medicine and Biology 31, 985-992 (1986) [4].

K. W. Leszczynski and S. Shalev, "A robust algorithm for contrast enhancement by local histogram modification," Imag. Vision Comp. 7 205-209 (1989) [9].

K. W. Leszczynski, S. Shalev, and N. S. Cosby, "The enhancement of radiotherapy verification images by an automated edge detection technique," Medical Physics 19 
$611-621(1992)[9,96,121] .$.

K. Leszczynski, S. Shalev, and G. Gluhchev, "Verification of radiotherapy treatments: Computerized analysis of the size and shape of radiation fields," Medical Physics 20, $687-694(1993)[126,151]$.

K. Leszczynski and S. Loose and P. Dunscombe, "Segmented chamfer matching for prescription-treatment image registration in radiotherapy," Physics in Medicine and Biology 40, 83-94 (1995) $[[9,151,166]$.

J. E. Marks, A. G. Haus, H. G. Sutton, and M. L. Griem, "Localization error in the radiotherapy of Hodgkins's disease and malignani lymphoma with extended mantle fields," Cancer 34, 83-90 (1974) [2,9].

D. Marr and E. Hildreth, "Theory of edge detection," Proceedings of Royal Society of London B 207, 187-217 (1980) [120,121].

G. Matheron, Random Sets and Integral Geometry (John Wiley \& Sons, New York, 1975) [103].

H. Meertens, J. Bijhold, and J. Strackee, "A method for measurement of field placement errors in digital portal images," Physics in Medicine and Biology 35, 1493-1502 (1990) [4].

J. Moseley and P. Munro, "A semiautomatic method of registration of portal images," Medical Physics 21, 551-558 (1994) [9,150]. 
P. Munro, J. A. Rawlinson, and A. Fenster, "A digital fluoroscopic imaging device for radiotherapy localization," Proceedings of SPIE 1090, 321-329 (1989) [4].

P. Munro, J. A. Rawlinson, and A. Fenster, "A digital fluoroscopic imaging device for radio herapy localization,” Int. J. Radiat. Oncol. Biol. Phys. 18, 64!-649 (1990) [86]. P. Munro, J. A. Rawlinson, and A. Fenster, "Therapy Imaging: A signal-to-noise analysis of fluoroscopic imaging systems for radiotherapy localization," Medical Physics 17, 763-772 (1990) [86].

U. Neitzel, I. Maack, and S. Gunther-Kohfahl, "Image quality of a digital chest radiography system based on a selenium detector," Medical Physics 21, 509-516 (1994) $[7,59]$.

J. Nelder and R. Mead, "A simplex method for function minimization," Computer Journal 7, 308-313 (1964) [151].

S. M. Pizer, E. P. Amburn, J. D. Austin, R. Cromartie, A. Geselowitz, T. Greer. B. H. Romeny, J. B. Zimmerman, and K. Zuiderveld, "Adaptive histogram equalization and its variations," Computer Vision, Graphics and Image Processing 39, 355-368 (1987) [9]. W. Press, S. Teukolshy, W. Vetterling, and B. Flannery, Numerical Recipes in C, 2nd edition (Cambridge University Press, Cambridge, UK, 1992) [135,151].

J. M. S. Prewitt, "Object enhancement and extraction," Picture Processing and Psychopictorics (B. S. Lipkin and A. Rosenfeld editors, Academic Press, New York, 1970) 
20, 99-102 [118].

T. Radcliffe, R. Rajapakshe, and S. Shalev, "Pseudosorrelation: A fast, robust, absolute, grey-level image alignment algorithm," Medical Physics 21, 761-679 (1994) [9].

L. E. Reinstein, H. I. Amols, P. J. Biggs, R. T. Droege, A. B. Filimonov, W. R. Lutz, and S. Shalev, AAPM Report No. 24 Radiotherapy Portal Imaging Quality(American Institute of Physics, New York, 1988) [3,97].

L. G. Roberts, "Machine perception of three dimensional solids," Optical and Electrooptical Information Processing (editors: B. S. Lipkin and A. Rosenfeld, Camgridge, Massachusetts, 1965) [118].

D. W. O. Rogers and A. F. Bielajew, "Monte Carlo techniques of electron and photon transport for radiation dosimetry," The Dosimetry of Ionizing Radiation III (editors: Kenneth R. Kase, Bengt E. Bjarngard, and Frank H. Attix, Academic Press, San Diego, California 1990) [30].

A. Rosenfeld and A. D. Kak, Digital Picture Processing (Academic Press, New York, 1982) [153].

J. A. Rowlands, G. DeCrescenzo and N. Araj, "X-ray imaging using amorphous selenium: Determination of x-ray sensitivity by pulse height spectroscopy," Medical Physics 19, 1065-1069 (1992) [66].

J. A. Rowlands and D. M. Hunter, "X-ray imaging using amorphous selenium: Pho- 
toinduced discharge (PID) readout for digital general radiography," Medical Physics 22. 1983-2005 (1995) $[7,59,173]$.

J. Serra, Image Analysis and Mathematical Morphology (Academic Press, London, UK 1982), 2nd edition [103].

S. Shalev, T. Lee, K. Lesczcynski, S. Cosby, and T. Chu, "Video techniques for on-line portal imaging, Computerized Medical Imaging and Graphics 13, 217-226 (1989) [4]. R. Shaw, "Some fundamental properties of xeroradiographic images," SPIE Proceedings $70,359-363$ (1975) [85].

P. Sprawls, Physical principles of medical imaging (Aspen, Gaithersburg, Maryland 1993), 2nd edition [85].

S. R. Sternberg, "Grayscale morphology," Computer Vision, Graphics and Image Processing 35 333-355 (1986) [103].

R. Swank, "Absorption and noise in x-ray phosphors," Journal of Applied Physics 44, 419:-4203 (1973) [21].

R. Swank, "Measurement of absorption and noise in an x-ray image intensifier," Journal of Applied Physics 45, 3673-3678 (1974) [21].

M. van Herk and H. Meertens, "A digital imaging system for portal verification," in Proceedings of the 9th International Conference on the Use of Computers in Radiation Therapy (Elsevier North-Holland, Amsterdam 1987) , 371-374 [4]. 
M. van Herk and H. Meertens, "A matrix ionization chamber imaging device for online patient setup verification during radiotherapy," Radiotherapy and Oncology 11, 369-378 (1988) [4].

M. van Herk, "The physics of a liquid filled ionization chamber with pulsed polarizing voltage," in Conference Records of the 10th International Conference on Conduction and Breakdown in Dielectric Liquid (IEEE, New York 1990), 126-130 [4].

M. van Herk, K. Gilhuijs, P. Williams, P. Cinquin, L. Cionini, and W. Swindel, "Role of electronic portal imaging in high dose/high precision radiotherapy," Radiotherapy and Oncology 21, 269-270 (1994) [9,150].

T. Villafana, "Modulation transfer function of a finite scanning microdensitometer slit," Medical Physics 2, 251-54 (1975) [27].

A. G. Visser, H. Huizenga, V. G. M. Althof, and B. N. Swanenburg, "Performance of a prototype fluoroscopic radiotherapy imaging system," Int. J. Radiat. Oncol. Biol. Phys. 18, 43-50 (1990) [4].

H. Wang and B. G. Fallone, "A robust algorithm for automatic segmentation and correlation of portal images," in Proceedings of the Joint Conference of The Canadian Organization of Medical Physicists and The Canadian Medical and Biological Engineering Society (Ottawa, Ontario, 1993), 44-45 [10].

H. Wang and B. G. Fallone, "A robust morphological algorithm for automatic radiation 
field-extraction and correlation of portal images," Medical Physics 21, 237-244 (1994) $[10,150]$

H. Wang and B. G. Fallone, "A sequential minimization technique in chamfer matching," in Proceedings of 3rd International Workshop On Electronic Portal Imaging (San Francisco, California 1994) [10].

H. Wang and B. G. Fallone, "A model-based algorithm for accurate extraction of the radiation field from double-exposure portal images," in Proceedings of 3rd International Workshop On Electronic Portal Imaging (San Francisco, California 1994) [10].

H. Wang and B. G. Fallone, "A mithematical model of radiation field edge localization," Medical Physics 22, 1107-1110 (1995) [10].

H. Wang and B. G. Fallone, "Matching treatment and prescription fields with a combined moment-chamfer matching technique," in Proceedings of 4 l st Annual Meeting Canadian Organization of Medical Physicists \& Canadian College of Physicists in Medicine with the Canadian Association of Radiologists, (Montreal, Quebec 1995) [10].

H. Wang and B. G. Fallone, "Model based localization of the radiation field border in double-exposure portal images," in Proceedings of 4/st Annual Meeting Canadian Organization of Medical Physicists \& Canadian College of Physicists in Medicine with the Canadian Association of Radiologists (Montreal, Quebec, 1995) [10].

H. Wang and B. G. Fallone, "A simplified technique for matching radiation and 
prescribed field," Radiology and Oncology 30, 58-65 (1996) [10].

H. Wang and B. G. Fallone, "Monte Carlo calculations of imaging characteristics of a-Se in electrostatic portal imaging," in Proceedings of the 42nd Annual Meeting of Canadian Organization of Medical Physicists (Vancouver, British Colombia, 1996), 286-289 [10]. H. Wang and B. G. Fallone, "A metal screen-amorphous selenium based image receptor in megavoltage porcal imaging (Abstract)," Medical Physics 23, 1130 (1996) [10].

L. Wolfe, L. Kalisher, and B. Considine, "Cobalt-60 treatment field verification by xeroradiography," American Journal of Roentgenology 118, 916-918 (1973) [7,59].

A. Zermeno, T. Kirby, R. Cowart, L. Marsh, and P. Ong, "Laser readout of electrostatic images," Proceedings of SPIE 173, $81-87$ (1979) [59].

W. Zhao and J. A. Rowlands, "X-ray imaging using amorphous selenium: Feasibility of a flat panel self-scanned detector for digital radiology," Medical Physics 22, 1595-1604 $(1995)[7,173]$.

S. Zhou and L. J. Verhey, "A robust method of multileaf collimator (MLC) leaf configuration verification," Physics in Medicine and Biology 39, 1929-1947 (1994) [151]. 